Fatty acid suppression of glial activation prevents central neuropathic pain after spinal cord injury

Marieta Georgieva ${ }^{1,3}$, Yuting Wei ${ }^{1}$, Maria Dumitrascuta ${ }^{1}$, Roger Pertwee $^{1}$, Nanna B. Finnerup ${ }^{2}$, Wenlong Huang ${ }^{1, *}$

${ }^{1}$ Institute of Medical Sciences, School of Medicine, Medical Sciences \& Nutrition, The University of Aberdeen, Foresterhill, Aberdeen, Scotland, AB25 2ZD, UK.

${ }^{2}$ Danish Pain Research Centre, Department of Clinical Medicine and Department of Neurology, Aarhus University Hospital, Aarhus, DK-8000, Denmark.

${ }^{3} 4 \mathrm{D}$ Pharma Research Ltd, Aberdeen, AB25 2ZS, UK

*Corresponding author information: Dr Wenlong Huang, PhD, MBBS, Institute of Medical

Sciences, University of Aberdeen, Foresterhill, Aberdeen, AB25 2ZD, UK; Telephone: +44 (0)1224

437290; Fax No.: +44 (0)1224 437465; Email: w.huang@ abdn.ac.uk

Page number of the entire manuscript $=44$; Number of figures $=9$; Number of tables $=2$

All authors have no conflicts of interest related to the research or the manuscript.

Part of the work was presented as abstracts for World Congress on Pain (Yokohama, 2016), International Congress on Neuropathic Pain (Gothenburg, 2017), and SfN Annual Meeting (San Diego, 2017). 
Introduction

Spinal cord injury (SCI) causes permanent impairment of motor and sensory functions. About 70-80\% of SCI patients suffer from different types of pain [21; 52], among which central neuropathic pain (CNP) is present in about $50 \%$ of patients $[7 ; 21 ; 52]$. At- and below-level SCI-CNP manifests as spontaneous, burning, stabbing, and/or electric shock-like sensation, stimulus-evoked allodynia and hyperalgesia, and often associates with paraesthesia and dysesthesia [20]. SCI-CNP is difficult to treat and significantly impacts on daily functioning/rehabilitation, often leading to depression [57]. No treatments in tolerable doses reliably produce satisfactory relief for a majority of SCI-CNP patients [20]; therefore, new therapeutic approaches are urgently needed.

Microglia and astrocytes contribute to the neuroinflammation underpinning SCI-CNP pathogenesis [25; 33]. They are activated chronically both at the injury site and in areas remote to injury, e.g. the lumbar L5 spinal cord, and are responsible for the development and maintenance of SCI-CNP [25]. They release glia-transmitters that activate receptors and ion channels in the neuronal membrane [25], and proinflammatory mediators including cytokines, chemokines, inducible nitric oxide synthase (iNOS), and prostaglandin E2 (PGE2) that induce CNP $[17 ; 28 ; 40 ; 60 ; 61]$. Collectively, these substances initiate and maintain the activation of a positive feed-forward cycle between neurons and glia at spinal and supraspinal regions, enabling persistent neuronal hyperexcitability post-SCI [25]. Therefore, targeting the activation of these cells presents a potential therapeutic strategy for SCI-CNP.

Docosahexaenoic acid (DHA), an omega-3 fatty acid crucial for the development and functioning of the nervous system, inhibits proinflammatory cytokine production in microglial cell lines [37]. When given as a single bolus intravenous (i.v.) injection combined with dietary supplementation, DHA conferred 
neuroprotection and improved locomotor function in rodent models of SCI [32; 36]. Other research showed that prophylactic dietary supplementation containing DHA and other fatty acids reduced thermal hyperalgesia in rats with SCI [19]. However, the therapeutic potential of DHA to prevent and treat SCICNP has yet to be clarified.

Here, we hypothesized that systemic DHA treatment would prevent SCI-CNP development when given (i.v.) immediately after SCI and followed by repeated i.v. administrations (acute regimen); and that it would abolish already developed CNP when given as repeated i.v. administrations at a chronic SCI (delayed regimen). By using a novel sensory profiling, including pain-related spontaneous and/or natural rodent behaviours [burrowing (affective), thigmotaxis (affective) and place escape/avoidance paradigm (PEAP, cognitive)] to mimic clinical practice to estimate human SCI-CNP, we showed that the acute regimen prevented the development of spontaneous/affective and cognitive pain behaviours coupled with significantly attenuated at-level mechanical hypersensitivity in a clinically relevant rat contusion SCI model [3]. We showed also that the delayed regimen partially abolished SCI-CNP. Using neurochemical and flow cytometry techniques, we showed that the mechanisms by which DHA prevents SCI-CNP include reduced microglial and astrocyte activations at both spinal and supra-spinal regions. Finally, we used quantitative real-time PCR (qPCR) to reveal the potential roles of the peroxisome proliferatoractivated receptor (PPAR), retinoid X receptor (RXR), and docosahexaenoyl ethanolamide (DHEA DHA's metabolite) in modulating microglial activation. 
Materials and Methods

\section{Ethical statement}

Experiments involving live animals were performed following UK Regulations (Animals Scientific Procedures Act, 1986) as well as IASP and NIH guidelines for using laboratory animals. Animal studies were approved by the local Ethics Committee of the University of Aberdeen. The ARRIVA guidelines were followed and an ARRIVA checklist was attached as a supplementary material.

\section{Study design}

Male Wistar rats (180-200 g; Charles River, UK) were housed (2-3 per cage) in a temperature/ humidity/noise-controlled environment, kept on a 12:12 hour light-dark cycle, and allowed to acclimatize for 48 hours after delivery. Standard rat chow and tap water were available ad libitum. The numbers of experimental groups and timelines for behavioural tests are detailed in Supplementary Table 1. Good laboratory practice $[39 ; 47]$ were followed to minimise bias (Supplementary Table 2). Sample size estimation is described in Supplementary Table 3. Behavioural tests were performed during day phase. Batches of subset behavioural experiments (2-3 per group) were used due to limited laboratory capacity and to achieve comparable timings between test animals.

\section{Contusion SCI surgery}

An established rat contusion model of SCI-CNP was adopted [2]. 2\% isoflurane (Abbot Laboratories, UK) was used for general anaesthesia. Buprenorphine $(0.3 \mathrm{mg} / \mathrm{kg}$ for post-surgery acute pain; Alstoe Animal Health, UK), Carprofen (50 mg/kg for post-surgery inflammation; Pfizer, UK), and Baytril (5 $\mathrm{mg} / \mathrm{kg}$ for post-surgery infection; Bayer Plc, UK) were administered subcutaneously (s.c.) before surgery. A T10 and partial T9 laminectomy was performed and followed by stabilising T8 and T11 spinous processes with a clamping apparatus and transferring the rat with the apparatus to the MASCIS 
impactor (rat model III; software 1.32.6; Rutgers University, USA). Next, a $10 \mathrm{~g}$ rod was released from a $12.5 \mathrm{~mm}$ height onto the exposed spinal cord and generated a vertical compression of the cord [2]. Finally, muscle and skin layers were sutured and rats were allowed to recover from anaesthesia before returning to home cages. Sham-operated animals underwent the same laminectomy procedure without contusion injury. Saline was given for 5 days $(5 \mathrm{ml} /$ day, s.c.) post-surgery. Bladders were manually expressed twice daily until self-voiding was resumed. Baytril was given during the first 5 days to minimize urinary infection.

\section{In vivo DHA administration}

DHA stocks (1 M; Sigma, UK) were prepared and stored at $-80^{\circ} \mathrm{C}$ [32]. For in vivo treatment, stocks were diluted with sterile $0.9 \%$ saline to desired concentrations immediately before intravenous (i.v.) tail vein injections. For the acute regimen study, DHA $(250 \mathrm{nmol} / \mathrm{kg})$ was administered 30 minutes postsurgery and thereafter every 3 days lasting for 6 weeks (Fig. 1A). The dosage choice was based on our previous studies [32]. For the delayed regimen study, DHA (250 nmol/kg) was administered at the beginning of week 4 post-surgery and thereafter every 3 days lasting for 4 weeks (Fig. 1B). For positive controls, Pregabalin was administered intraperitoneally (30 mg/kg; Pfizer, UK) 45 minutes before painrelated behavioural assessment [2] at week 3 and week 6 for the acute regimen study and at week 6 for the delayed regimen study.

\section{A novel sensory profiling approach to assess SCI-CNP}

The approach consists of evoked mechanical hypersensitivity measurement at- and below-injury level (sensory component of pain). It also consists of pain-related spontaneous and/or natural rodent behaviours i.e. burrowing (affective component of pain), thigmotactic (affective component of pain) and PEAP (cognitive component of pain) behaviours, which were previously reported by us $[2 ; 3 ; 30 ; 31]$. 


\section{Spinal reflex-based below-level mechanical hypersensitivity}

Below-level mechanical hypersensitivity was measured using an electronic von Frey device with 0.5 $\mathrm{mm}^{2}$ probe tip area (Somedic Sales AB, Sweden) as previously described [31]. In three consecutive days rats were acclimatized to the testing room for 45 minutes and then habituated to Plexiglas boxes for 60 minutes until exploratory behaviour ceased. The same Plexiglas box was used for the same rat throughout the duration of the study. Two consecutive baseline measurements were taken before surgery. The probe was applied to the mid-lateral plantar surface of the hindpaw, delivering an increasing force (8-15 g/s) until active paw withdrawal. Both hindpaws were tested 5 times with a one-minute interval and results were pooled. An average of data from 10 trials per rat was calculated as the withdrawal threshold in response to punctate static mechanical stimulation.

\section{Brainstem response-based below-and at-level mechanical hypersensitivity}

Brainstem responses (licking, guarding, vocalizing, jumping or biting) to below- and at-level mechanical stimuli were measured as previously described [2; 3]. Acclimatization, habituation, and baseline assessments were performed as above. For at-level brainstem responses, nylon von-Frey filaments (Bioseb, USA) with increasing diameters exerting different forces - 0.07, 0.16, 0.4, 0.6, 1.4, 2, 4, 6, and $8 \mathrm{~g}$ (cut off) were applied $2 \mathrm{~cm}$ laterally to the SCI site, starting with $1.4 \mathrm{~g}$ filament. A total of 9 filament applications, 30 seconds apart, were performed per rat. For below-level brainstem responses, nylon vonFrey filaments ranging 1, 1.4, 2, 4, 6, 8, 10, 15, $26 \mathrm{~g}$ (cut off) were used. The filaments, starting with 6 $\mathrm{g}$, were applied to the mid-lateral plantar surface of both hindpaws for 5 times each; a total of 10 filament applications, 30 seconds apart, were performed per hindpaw and bilateral results were pooled. An 'updown' method [9] was used to calculate the 50\% withdrawal threshold in response to at- and below-level mechanical stimulations. 


\section{Spontaneous and natural burrowing behaviour}

Burrowing, an evolutionally conserved rodent behaviour, is easy to measure objectively by weighing the amount of gravel left in the burrow after testing. Plastic tubes were custom-made (32-cm-long and 10$\mathrm{cm}$-diameter) with one end sealed and the other opened as entrance [raised by $6 \mathrm{~cm}$ to prevent gravel $(5$ mm pea shingle; B\&Q, UK) loss]. Before each burrowing training or assessment, rats were habituated to the testing room and cages (30 minutes each) with dim lighting (5 lux). Before baseline measurements, rats were trained to burrow in pairs in one cage with a tube containing $2500 \mathrm{~g}$ of gravel for 2 hours. The next day, rats that performed well were re-grouped with those that performed less well in the first training, to facilitate peer learning. Two baseline assessments were conducted per rat in two consecutive days. Burrowing was assessed weekly post-surgery starting from the second week, when plantar stepping was re-established (Supplementary Fig. 1).

\section{Spontaneous and natural thigmotactic behaviour}

This is based on natural behaviours of rodents when they enter an open field to which they have not previously been exposed. Thus, rats were introduced for first time to an open field arena (100-cm-long and 100-cm-wide) in dim lighting (12 lux) at week 6 and week 7 post-surgery for the two regimen studies respectively. Spontaneous movement of each rat in the arena was recorded for 15 minutes with a highlysensitive camera (Tracksys Ltd., UK) and movement tracks were analysed using EthoVision XT (v10, Noldus, The Netherlands). The duration spent in and frequency of entry to the virtual inner zone (40-cmlong and 40-cm-wide) were calculated.

\section{Natural PEAP behaviour}

This is based on natural behaviours of rodents in which they prefer hiding in the dark than exposing to bright conditions. The paradigm was custom-made with acrylic box (60-cm-long, 30-cm-wide, 30-cmhigh; painted into half white and half black; 12 lux lighting). Rats were allowed to acclimatize in the 
testing room for 30 minutes before testing. Then each rat was introduced to the centre of the box and allowed a 5-minute habituation. A von Frey filament (2 g), a choice based on our previous studies [2; 3], was next applied every 15 seconds either on the dorsal thorax ( $2 \mathrm{~cm}$ laterally to the injury site) when the rat was in the black zone or on skull vertex when it was in the white zone, for 30 minutes during which free movement was allowed. Free movements of each rat were recorded using EthoVision, enabling the calculation of the cumulative time spent in and number of crossings to the white zone.

\section{Immunohistochemistry and quantitative analysis}

At the end of the behavioural studies, animals ( $n=5-6$ per group) were perfused with $4 \%$ paraformaldehyde. Brains and spinal cords (L5 and injury epicenter) from SCI rats and naïves/shams (cords with equivalent level to injury epicenter) were collected. Tissues were post-fixed, cryoprotected in 30\% sucrose in phosphate-buffered saline (PBS), and embedded in OCT. Cryostat transverse sections $(14 \mu \mathrm{m})$ were cut, thaw-mounted onto Superfrost glass-slides, and incubated with primary antibodies: rabbit anti-Iba-1 (1:700; Wako, Japan), mouse anti-GFAP (1:1000; Merck Millipore, Germany) or rabbit anti-laminin (1:400; Abcam, UK). Sections were then incubated with appropriate secondary antibodies: goat/donkey anti-rabbit Alexa Fluor ${ }^{\circledR} 488$ or anti-mouse Alexa Fluor ${ }^{\circledR} 568$ (all 1:400; Fisher Scientific, UK). Phospho-p38 (pp-38) expression in microglia was investigated as previously reported [30; 31]. Sections were incubated with rabbit anti-pp-38 primary antibody (1:500; Cell Signalling, USA) followed by incubation with goat anti-rabbit biotin (1:400; Vector Laboratories, USA), which was then amplified using ABC Kit (Vector Laboratories, USA) and TSA Biotin System (Perkin Elmer, UK). Sections were then incubated with Extra-Avidin FITC (1:500; Fisher Scientific, UK), followed by incubations with rabbit anti-Iba-1 (1:700) and goat anti-rabbit Alexa Fluor ${ }^{\circledR} 68$ (1:400). All sections were triple-washed with PBS following any antibody incubation (primary - overnight, secondary - 2 hours, all at room temperature) and had a final nuclei-staining with DAPI before mounting in PBS-glycerol (1:8). 
Staining was visualized using a Nikon Ti-E fluorescence microscope. Images were taken at 20x objective magnification from 4 randomly selected areas in L5 and epicenter dorsal horns and the anterior cingulate cortex (ACC), from 5 serial sections per tissue type. Microglial activation was analysed as we previously described [30; 31]. Iba-1 immunoreactive cells with 'reactive' morphology (the process length is twice shorter than that of the soma diameter) were counted and expressed as the number per $50,000 \mu \mathrm{m}^{2}$. Double-immunoreactive (pp-38+/Iba-1+) cells were counted and expressed as a percentage of the total of Iba-1+ microglia. Astrocyte activation was determined by analysing the intensity of GFAP immunoreactivity: images were converted into 8-bit using ImageJ and a threshold was applied to render the staining as a binary image in black and empty spaces in white; then the area occupied by GFAP staining was calculated. Images from the ACC were also analysed for the numbers of Iba-1 immunoreactive cells and GFAP intensity. The person who conducted these analyses was blinded to treatment groups.

For the spinal cord histology, we used transverse sections from a 5-mm long spinal cord segment which contained the lesion epicenter in the middle. Thus, a series of transverse sections with $14 \mu \mathrm{m}$ thickness were cut from the rostral end of this segment towards caudal until the finish of sectioning. Due to the cavity formation in the lesion epicenter which destroyed the dorsal horn, we selected transverse sections approximately $2000 \mu \mathrm{m}$ both rostral and caudal to the middle of the segment. In these sections, despite still having small cavities, the superficial dorsal horns were largely intact thus allowing us to conduct imaging and image analysis for microglia and astrocytes. The right and left dorsal horn data were averaged per section. The rostral and caudal histological measurements were then averaged per rat.

\section{Eriochrome-cyanine staining and analysis}

Eriochrome cyanine (EC) staining was used to analyze the amount of tissue sparing i.e. spared white matter myelin from the spinal cord tissue of injured animals ( $n=4-5$ per group). Transverse $14 \mu \mathrm{m}$ thick 
cryostat sections approximately $2000 \mu \mathrm{m}$ away from the epicenter in both rostral and caudal directions, were selected for this purpose. Staining procedures were previously described [53]. Briefly, sections were dried overnight at room temperature, and then were placed in fresh acetone for 5 minutes, removed, and air dried for 20 minutes. Sections were incubated in EC solution (0.2 g eriochrome cyanine R, $0.5 \mathrm{ml}$

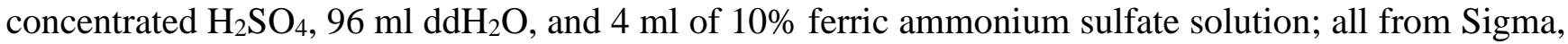
UK) for 30 minutes at room temperature and then washed in $\mathrm{ddH}_{2} \mathrm{O}$. The sections were then differentiated in 5\% iron alum (Sigma, UK) for around 15 minutes until the grey matter became visible. This was followed by differentiation in a borax-ferricyanide solution ( $1 \mathrm{~g}$ disodium tetraborate decahydrate, 1.25

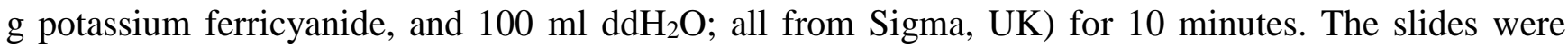
dehydrated by graded 70\%, 95\% and 100\% ethanol, cleared through xylene and mounted by Neo-mount (Sigma, UK). To calculate the area of spared myelin, bright-field images of three EC-stained sections per rat (rostral, epicenter and caudal) were taken by a Nikon DS-U3 camera. Tissue sparing was quantified by outlining areas with light and dark blue staining, analyzed by ImageJ, and calculated by dividing the spared white matter area (WMA) by the spinal cord transverse cross-sectional area (CSA).

\section{Glial cultures, in vitro DHA treatment and immunocytochemistry}

Wistar rat pups (postnatal days 3-5) were used and mixed microglial and astrocyte cultures were prepared as we previously described [22]. Briefly, isolated cortical tissue was mechanically and chemically dissociated and then cells were seeded onto $60 \mathrm{~mm}$ poly-D-lysine (PDL; Sigma, UK) coated culture dishes in Dulbecco's Modified Eagle's Medium (DMEM) supplemented with 15\% heat inactivated foetal bovine serum (FBS) (Invitrogen, UK). After 4 days in culture, microglia were harvested and plated onto uncoated glass coverslips. The astrocyte layer was left to incubate for another 48 hours and then cells harvested by incubation with $0.25 \%$ Trypsin-EDTA solution (Sigma, UK). Once astrocytes were detached, the cell suspension was centrifuged at $1500 \mathrm{rpm}$ for 10 minutes, and then cells were counted, resuspended and plated onto PDL-coated coverslips. 
DHA stock was diluted with $25 \mathrm{ml}$ serum free DMEM to obtain $200 \mu \mathrm{M}$ stock solution for in vitro simultaneous treatment or delayed treatment (ST vs DT). For ST experiments, cultured cells were cotreated with DHA and Lipopolysaccharides (LPS, $10 \mu \mathrm{g} / \mathrm{ml}$; Sigma, UK) for 4 hours. For DT experiments, cells were pre-activated by LPS for 4 hours and then DHA was given for another 4 hours. To determine the optimal DHA concentration, cultured cells were first treated with $0.8,4,8$ or $32 \mu \mathrm{M}$ DHA [48] during the ST experiments. $0.8 \mu \mathrm{M}$ was found as the optimal concentration and was used in subsequent ST/DT experiments. Cells were either fixed with 4\% PFA for immunocytochemistry, lysed with RLT buffer (Qiagen, UK) for q-PCR, or processed for flow cytometry. Culture media were collected for the Greiss assay analysis (Supplementary Methods).

For immunocytochemical analysis, cells were incubated with primary antibodies: rabbit anti-Iba-1 (1:1000), mouse anti-GFAP (1:1000) and mouse anti-iNOS/NOS type II (1:200; BD Bioscience, UK), followed by incubation with goat/donkey secondary antibodies: anti-rabbit Alexa Fluor®488, anti-mouse Alexa Fluor®568, or anti-mouse IgG2a Alexa Fluor®555 (all 1:400; Fisher Scientific, UK). Images from five random areas per coverslip were taken at 20x objective magnification. Microglial and astrocyte morphologies were qualitatively assessed using bright-field images. Microglial/astrocyte culture purity was determined by calculating the percentage of Iba-1 or GFAP immunoreactive cells in the total of DAPI-stained cells. Coverslips with purities less than $95 \%$ were excluded from analysis. iNOS expression was determined by calculating the percentage of iNOS/Iba-1 double-immunoreactive cells in the total of Iba-1 immunoreactive cells. We analysed the GFAP staining intensity as described above. The person who performed the analyses was blinded to treatment groups.

\section{Flow cytometry analysis}

In vivo flow cytometry 
Fresh-frozen cord tissues were collected at the end of behavioural studies and assessed for microgliosis, known to be correlated with neuropathic pain behaviours, using flow cytometry as previously reported [5]. Segments of L5 and epicenter cords ( $n=4-5$ per group) were immediately frozen on dry ice after harvesting and stored at $-80^{\circ} \mathrm{C}$. Samples were thawed on ice, weighed $(\mathrm{mg})$, and then enzymatically digested with collagenase type IV $\left(1 \mathrm{ml} ; 0.5 \mathrm{mg} / \mathrm{ml}\right.$ in PBS; Sigma, UK) for 30 minutes at $37^{\circ} \mathrm{C}$. The cell suspension was passed through a $40 \mu \mathrm{m}$ cell strainer (BD Falcon, UK), followed by centrifuging at 400 rpm for 10 minutes and resuspending in $10 \%$ normal goat serum and $1 \% 2 \mu \mathrm{M}$ EDTA for 15 minutes. Each sample was diluted in FACS buffer to contain a minimum of $5 \times 10^{6}$ cells and incubated with: CD172a-FITC (clone-ED9; microglial marker; Bio-Rad, UK), CD11b/c-APC (clone-REA325; microglial marker; Miltenyi Biotec, UK), CD45-Vioblue (clone-REA504; general macrophage marker; Miltenyi Biotec, UK), all at 1:10 dilution for 45 minutes at room temperature. Samples were then washed and resuspended with FACS buffer, followed by analysis using flow cytometry machine BD Fortessa (BD, UK) $\left(5 \times 10^{5}\right.$ events per sample) and FlowJo software (FLOWJO, USA). Data were presented as numbers of ED9+/CD11b/c+ cells per mg of spinal cord tissue.

\section{$\underline{\text { In vitro flow cytometry }}$}

We also assessed microglial activation in vitro using flow cytometry. Cells from the ST/DT experiments were detached using StemPro Accutase Cell Dissociation Reagent (Fisher Scientific, UK), and then fixed with $2 \%$ PFA for 10 minutes. Cell suspension was then centrifuged at $1500 \mathrm{rpm}$ for 10 minutes. Cell pellet was washed with PBS, spun twice, and resuspended in $100 \mu$ FACS buffer. Cells were incubated with CD11b/c-FITC (clone-REA325) and CD86-PE (clone-24F) (Miltenyi Biotec, UK) at 1:10 dilution $\left(10^{6}\right.$ cells $\left./ 50 \mu \mathrm{l}\right)$ for 45 minutes at room temperature. Samples were analysed using BD Fortessa (a minimum of 10,000 events) and FlowJo software. Data were expressed as percentages of CD11b/c+/CD86+ cells in total CD11b/c+ cells. 


\section{q-PCR analysis}

\section{In vivo qPCR for spinal cord dorsal horn tissues}

To assess relative mRNA expression levels of proinflammatory mediators iNOS, tumour necrosis factor$\alpha(\mathrm{TNF}-\alpha)$, interleukin-1 $\beta$ (IL-1 $\beta$ ), and IL-6 in vivo, spinal cord dorsal horn tissues at L5 level and from lesion site segment containing the epicenter ( $n=4-5$ per group) were freshly dissected out using the open book method that we previously reported [15] and processed for q-PCR as we previously described [43]. Tissues were dissected approximately $1.5-2 \mathrm{~mm}$ away both rostral and caudally to the epicenter, where dorsal horns were relatively preserved. Left/right and rostral/caudal tissues from each animal were pooled. Tissues were immediately frozen on dry ice after harvesting and stored at $-80^{\circ} \mathrm{C}$ until use. Frozen spinal cord tissue was homogenized in $1 \mathrm{ml}$ Trizol (Fisher Scientific, UK) and separated into phases by using $200 \mu \mathrm{l}$ chloroform (Sigma, UK). The aqueous phase was transferred in a fresh tube and cleaned up by using RNeasy Mini Kit (Qiagen, UK), following the manufacturer's instructions. The concentration of eluted RNA was quantified using Nano Drop 1000 spectrophotometer (Fisher Scientific, UK). The qScript cDNA SuperMix (VWR, UK) was used to synthesise cDNA from RNA samples. A mixture of 4 $\mu 1$ cDNA SuperMix and $16 \mu$ RNA samples was prepared for cDNA synthesis. RNA samples were diluted to a final concentration of $100 \mathrm{ng} / \mu \mathrm{l}$ RA with RNA-free water. cDNA synthesis was performed by a thermal cycler according to the manufacturer's instructions and the samples were stored at $-20^{\circ} \mathrm{C}$ until further use. All used primers (Sigma, UK) used are listed in Table 1 [43]. A total of $15 \mu 1$ reaction solution, including $3 \mu \mathrm{l}$ cDNA sample and $12 \mu \mathrm{l}$ gene-specific master mix, was loaded in triplicates for each biological sample in 384 well plate. cT values were obtained by Light Cycler 480 II thermal cycler and Cycler 480SW1.5.1 software (Roche, UK). The relative mRNA expression of each gene was calculated and expressed as fold change using the $2^{-\Delta \Delta \mathrm{CT}}$ method [50]. Data were normalized against the reference gene YWHAZ. 
$\underline{\text { In vitro } q P C R}$

To assess relative mRNA expression levels of proinflammatory mediators iNOS, TNF- $\alpha$, IL-1 $\beta$, and IL6 in microglia and astrocytes from the ST/DT experiments, q-PCR was performed, as previously described [43]. Following a sterile PBS wash, a total of 500 ng RNA from individual samples was extracted using RNeasy Mini Kit (Qiagen, UK), following manufacturer's instructions. The RNA concentration was confirmed using Nano Drop 1000 spectrophotometer. cDNA synthesis, qPCR methods and the relevant analysis were performed in the same manner, as described above.

\section{PPAR and RXR antagonist treatment of microglia in vitro}

DHA is a well-known natural ligand of the PPAR and RXR receptors $[12 ; 14 ; 24 ; 35 ; 63]$, which have been previously shown to have a regulatory role in various inflammatory- and immunity-related processes $[29 ; 54 ; 58]$. To investigate the potential mechanisms through which DHA attenuates the activation of microglia in vitro, we treated the microglial cells with potent PPAR and RXR antagonists prior to simultaneous treatment with LPS and 0.8 $\mu$ M DHA. PPAR $\gamma$ antagonist GW9662 (10 mM; Sigma, UK) and RXR antagonist HX531 (2 mM; Tocris, UK) initially reconstituted in dimethyl sulfoxide (DMSO) (Sigma, UK) were prepared to final working concentration of $10 \mu \mathrm{M}$ in serum free DMEM medium, as described previously [23;59]. After the cells were pre-treated for 4 hours with one of the antagonists or a combination of both, the culture medium was replaced with a combination of $10 \mu \mathrm{g} / \mathrm{ml}$ LPS and $0.8 \mu \mathrm{M}$ DHA treatment for 4 hours as described previously. At the end of the treatments the cells were processed for the analysis of gene expression profiles of proinflammatory mediators, as described in the main methods section.

\section{DHA ethanolamide treatment of microglia in vitro}

To gain further insight of the mechanisms of DHA effects on microglial modulation, we investigated whether treatment with the DHA metabolite DHEA would also lead to a decrease in the activation of 
microglia in vitro. The rationales behind assessing the effects of DHEA are that firstly, the levels of this downstream substrate have been shown previously to be increased in the brain and the spinal cord following consumption or administration of omega-3 fatty acids [19], secondly DHEA also possesses anti-inflammatory properties and enhances the beneficial effect of DHA [4; 19; 41], and finally DHEA also binds to the PPAR, RXR and CB1/2 cannabinoid receptors expressed by microglia [4; 6; 19; 41]. DHEA (Cayman Chemicals, Cambridge Bioscience, UK) was diluted to stock concentration of $10 \mathrm{mM}$ in the same way as described in the main text for DHA and further prepared in serum free DMEM to a working concentration of $5 \mu \mathrm{M}$ or $10 \mu \mathrm{M}$. The choice of these concentrations was based on previous studies [6]. The microglial cells were treated for 4 hours with either LPS $10 \mu \mathrm{g} / \mathrm{ml}$, a combination of LPS with $5 \mu \mathrm{M}$ DHEA, or LPS and $10 \mu \mathrm{M}$ DHEA, following which the cells were processed for the analysis of gene expression profiles of proinflammatory mediators, as described in the main methods section.

\section{Statistical analysis}

Statistical analyses were performed using Prism7 Software (La Jolla, USA) and SPSS Version 25 (IBM, USA). All data were expressed as mean \pm standard error of the mean (SEM). The primary outcomes for behavioural experiments are shown in Supplementary Table 1. For below- and at-level mechanical hypersensitivity, burrowing, and locomotor function assays, a two-way repeated-measures (between- and within-subjects) ANOVA was used to determine the main effects of independent variables namely "treatment" (DHA vs vehicle) and "time" (weeks post-injury). "Treatment" is a between-subjects variable, while "time" is a within-subjects variable. Since naïve animals and shams did not receive DHA or vehicle treatment, we did not include the naïve/sham groups in the two-way repeated-measures ANOVA analysis. However, we used one-way ANOVA for the naïve, sham, vehicle, DHA and pregabalin groups at each individual time (week), followed by the Tukey-Kramer post hoc multicomparison adjustment to calculate the significant levels. For thigmotaxis and PEAP, one-way ANOVA followed by the Tukey-Kramer post hoc multi-comparison adjustment were used to determine the main 
effect and significance levels. The box/scatter plots for thigmotaxis/PEAP data were prepared using OriginPro 2018 (OriginLab, USA).

Histological data were tested for normality first as we previously described [30; 31]. Parametric (two tailed unpaired student $t$-test) and nonparametric (Mann-Whitney test) tests were then used accordingly. For immunocytochemical, flow cytometry, and nitrite release analyses, unpaired student $t$-test was used. One-way ANOVA was used to determine the effect of DHA concentration on cultured microglia and astrocytes. For qPCR, statistical significance was calculated as we previously described, i.e. by t-tests in $\mathrm{R}$ (two-sided, Welch's $\mathrm{t}$ test) on the $\Delta \mathrm{Ct}$ values, followed by adjusting the $\mathrm{p}$ values using the false discovery rate correction [43]. We considered differences to be statistically significant when $\mathrm{p}<0.05$. 
Results

Information for group sizes and primary outcome measures of the in vivo experiments is detailed in Supplementary Table 1. Animal exclusion from the in vivo experiments is detailed underneath Supplementary Table 2 .

\section{Systemic DHA treatment attenuates spinal reflex-based below-level mechanical hypersensitivity}

We measured the left and right hindpaw withdrawal thresholds in response to mechanical stimuli, which were then averaged for each rat. Our analysis showed significant main effects of treatment on hindpaw mechanical hypersensitivity in both studies (acute regimen: $F_{(1,27)}=75.735, p=0.0001$, Fig. 1C; delayed regimen: $F_{(1,26)}=57.221, p=0.001$, Fig. 1D; two-way repeated-measures ANOVA). We did not find significant main effects of time on hindpaw mechanical hypersensitivity in both studies (acute regimen:

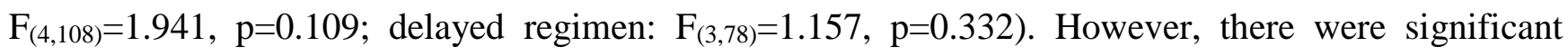
treatment with time interactions in both studies (acute regimen: $\mathrm{F}_{(4,108)}=5.323, \mathrm{p}=0.001$, delayed regimen: $\left.F_{(3,78)}=10.513, p=0.0001\right)$. Significant increases in withdrawal thresholds were observed in DHA-treated rats when compared to vehicle-treated rats (acute regimen/weeks 2-6: $\mathrm{p}<0.0021$; delayed regimen/weeks 5-7: $\mathrm{p}<0.0062$; Tukey-Kramer post hoc). However, the increased withdrawal thresholds of DHA-treated rats didn't reach to those of naïves (Fig. 1C, 1D).

\section{Systemic DHA treatment attenuates brainstem response-based mechanical hypersensitivity}

Our analysis revealed significant main effects of treatment on brainstem response (licking, guarding, vocalizing, jumping or biting)-based at-level mechanical hypersensitivity in both studies (acute regimen: $F_{(1,27)}=52.031, p=0.00001$, Fig. 1E; delayed regimen: $F_{(1,26)}=21.707, p=0.0001$, Fig. 1F; two-way repeated-measures ANOVA). We also found significant main effects of time on brainstem response in 
both studies (acute regimen: $\mathrm{F}_{(4,108)}=2.405$, $\mathrm{p}=0.045$, delayed regimen: $\mathrm{F}_{(3,78)}=11.782, \mathrm{p}=0.001$; two-way repeated-measures ANOVA). Moreover, there were significant treatment with time interactions in both studies (acute regimen: $\mathrm{F}_{(4,108)}=6.385, \mathrm{p}=0.0001$, delayed regimen: $\mathrm{F}_{(3,78)}=17.28, \mathrm{p}=0.0001$ ). The mean at-level $50 \%$ pain thresholds were significantly higher in DHA-treated rats than vehicle-control rats (acute regimen/weeks 2-6: $\mathrm{p}<0.022$; delayed regimen/weeks 5-7: $\mathrm{p}<0.028$; Tukey-Kramer post hoc), but lower than naïves (Fig. 1E, 1F). Pregabalin-controls showed comparable (no statistical difference) brainstem responses to at-level mechanical stimulation to DHA-treated rats (Table 2). The result showing no difference in brainstem responses to hindpaw mechanical stimulation (Fig. $1 \mathrm{G}, 1 \mathrm{H}$ ) was consistent with our previous findings [2].

It is important to note that we assessed brainstem response-based at-level mechanical hypersensitivity one day before burrowing, thigmotaxis, or PEAP testing throughout the two regimen studies. We observed at-level mechanical hypersensitivity in all SCI rats (versus naïves, Fig. 1) before they were assessed next day for one of the three complex behaviours. However, at-level mechanical hypersensitivity was attenuated in rats receiving systemic DHA treatments (versus vehicle-controls; weeks $2-6 /$ Fig. 1E and weeks 5-7/Fig. 1F) in the two regimen studies one day before testing for the complex behaviours.

\section{Systemic DHA treatment prevents deficit in pain-related spontaneous burrowing behaviour}

Burrowing is an ethologically relevant pain-related behaviour, reproducible in different lab settings [1; $30 ; 49 ; 56]$. The behavioural construct is that pain would interfere with a rat's motivation to burrow, which can be measured by the amount of gravel displaced from a tube. At baseline, no difference was measured in gravel displacement in the two regimen studies (Fig. 2A, 2B). We first demonstrated a significant main effect of treatment on burrowing performances by the acute regimen (weeks 2-6: $\mathrm{F}_{(1,27)}=109.338, \mathrm{p}=0.000001$, two-way repeated-measures ANOVA, Fig. 2A). There was also a 
significant main effect of time on burrowing performances by the acute regimen $\left(\mathrm{F}_{(4,108)}=5.214\right.$, $\mathrm{p}=0.001)$. However, there was no significant treatment with time interaction $\left(\mathrm{F}_{(4,108)}=0.957, \mathrm{p}=0.434\right)$. Compared to naïves, vehicle-treated rats developed significant burrowing deficits from week 2 which were maintained up to week 6 post-surgery (Fig. 2A). In contrast, DHA-treated rats displaced significantly more gravel than vehicle-treated rats (e.g. week 4: $1696.2 \pm 5.8$ g vs $840.6 \pm 7.8 \mathrm{~g}$, $\mathrm{p}=0.00044$, Tukey-Kramer post hoc, Fig. 2A). Strikingly, DHA-treated rats and naïves in the acute regimen study demonstrated comparable burrowing performances ( $\mathrm{p}=0.18$ to 0.99 , Tukey-Kramer post hoc). Next, our analysis revealed a significant main effect of treatment on burrowing performances by the delayed regimen (weeks 4-7: $\mathrm{F}_{(1,26)}=455.212, \mathrm{p}=0.0001$, two-way repeated-measures ANOVA, Fig. 2B). However, there were no significant main effects of time on burrowing performance $\left(\mathrm{F}_{(3,78)}=2.492\right.$, $\mathrm{p}=0.066)$, nor any significant treatment with time interaction $\left(\mathrm{F}_{(3,78)}=0.467, \mathrm{p}=0.706\right)$. Vehicle-control maintained significant burrowing deficits during this period when compared to naïves. Delayed DHA treatment significantly attenuated the reduced burrowing performances developed before treatment when compared to vehicle-control (e.g. week 6: $1366.8 \pm 5.4 \mathrm{~g}$ vs $891.3 \pm 6.1 \mathrm{~g}, \mathrm{p}=0.032$, Tukey-Kramer post hoc, Fig. 2B), but such attenuated performances were still lower than naïves ( $\mathrm{p}=0.026$ to 0.0042 , TukeyKramer post hoc). Pregabalin-controls produced comparable burrowing performances in both studies to DHA-treated rats (Table 2). These data establish that systemic DHA treatment prevents the development of affective/motivational aspects of SCI-CNP shown by burrowing behaviour.

\section{Systemic DHA treatment prevents pain-related spontaneous thigmotactic behaviour}

We next tested thigmotaxis - another ethologically relevant pain-related rodent behaviour $[5 ; 30 ; 31 ; 42]$. As prey animals, rats prefer to adhere to the perimeter of a novel arena, conflicting with their drive to explore arena centre [30]. The behavioural construct is that if a rat is in pain it would be in its survival interest to decrease its exposure to predators. We assessed thigmotaxis by analyzing spontaneous free 
movements of rats in an open-field arena. Since this is a novel test that could be affected by repeated testing [11], rats were only tested once at week 6 and week 7 for the two studies respectively. In the acute regimen study, we found significant treatment effects on inner zone cumulative time and entry frequency (time: $\mathrm{F}_{(2,36)}=39.8, \mathrm{p}=0.0075$, Fig. $3 \mathrm{~A}, 3 \mathrm{~B}, 3 \mathrm{C}, 3 \mathrm{D}$; frequency: $\mathrm{F}_{(2,36)}=11.8, \mathrm{p}=0.0050$, Supplementary Fig. 2a; one-way ANOVA). Vehicle-control rats had significantly reduced cumulative time and entry frequency at week 6 post-surgery versus naïves (time: $p=0.00042$, Fig. 3A, 3B, 3D; frequency: $p=0.0002$, Supplementary Fig. 2a; Tukey-Kramer post hoc). DHA treatment starting immediately post-surgery caused significant increases in cumulative time and entry frequency at week 6 when compared to vehicletreated rats (time: $37.2 \pm 7.2$ s vs $11.2 \pm 3.4$ s, p=0.0025, Fig. 3B, 3C, 3D; frequency: p=0.003, Supplementary Fig. 2a; Tukey-Kramer post hoc); cumulative time by DHA treatment was markedly similar to naïves ( $\mathrm{p}=0.199$, Fig. 3A, 3C, 3D, Tukey-Kramer post hoc). We also found significant treatment effects on thigmotactic behaviour by the delayed regimen (time: $F_{(2,35)}=25.21$, $p=0.014$, Fig. 3E, 3F, 3G, 3H; frequency: $\mathrm{F}_{(2,35)}=7.1, \mathrm{p}=0.0029$, Supplementary Fig. 2b; one-way ANOVA). Rats receiving vehicle treatment had significantly reduced cumulative time and entry frequency at week 7 post-surgery versus naïves (time: p=0.0090, Fig. 3E, 3F, 3H; frequency: p=0.0041, Supplementary Fig. 2b; Tukey-Kramer post hoc). DHA treatment starting week 4 post-surgery significantly improved cumulative time and entry frequency at week 7 in contrast to vehicle-control (time: $33 \pm 5.3 \mathrm{~s}$ vs $16.2 \pm$ 4.5 s, p=0.038, Fig. 3F, 3G, 3H; frequency: p=0.042, Supplementary Fig. 2b; Tukey-Kramer post hoc); however, the attenuated cumulative time following DHA treatment was less than naïves $(\mathrm{p}=0.013$, Tukey-Kramer post hoc). There was no significant difference in the total distance travelled in the open field among different experimental groups in both studies (data not shown). Pregabalin-controls in both studies had comparable thigmotactic behaviour to DHA-treated rats (Table 2). These data suggest that systemic DHA treatment can prevent SCI-CNP at affective/decision-making levels shown by thigmotactic behaviour. 


\section{Systemic DHA treatment prevents pain-related PEAP behaviour}

We previously assessed cortical processing of SCI-CNP using PEAP [2;3], where rats naturally prefer to hide in the dark (non-aversive) and avoid exposure to bright conditions (aversive). The behavioural construct is that if a rat feels pain (e.g. due to painful stimulation received at the injured area) in a dark arena, it would rather choose to enter a bright arena to avoid that stimulation. As a novel test [2;3], PEAP behaviour was only assessed at week 6 and week 7 for the two studies respectively, by analysing free movements of rats between the white/black zones of PEAP. We found significant treatment effects on white zone cumulative time and crossing number by the acute regimen (time: $\mathrm{F}_{(2,36)}=30.61, \mathrm{p}=0.0026$, Fig. 4A, 4B, 4C, 4D; number: $\mathrm{F}_{(2,36)}=5.78, \mathrm{p}=0.0083$, Supplementary Fig. 3a; one-way ANOVA). Compared to naïves, cumulative time and crossing number at week 6 post-surgery were significantly increased in vehicle-treated rats (time: $p=0.0017$, Fig. 4A, 4B, 4D; number: $p=0.0010$, Supplementary Fig. 3a; Tukey-Kramer post hoc). Strikingly, rats receiving DHA treatment starting immediately postsurgery significantly reduced their cumulative time ( $\sim 47 \%)$ and crossing number $(\sim 35 \%)$ when compared to vehicle-control (time: $\mathrm{p}=0.0028$, Fig. 4B, 4C, 4D; number: $\mathrm{p}=0.0059$, Supplementary Fig. 3a; TukeyKramer post hoc); their cumulative time was markedly similar to naïves ( $\mathrm{p}=0.93$, Tukey-Kramer post hoc, Fig. 4A, 4C, 4D). We also found significant treatment effect on PEAP behaviour by the delayed regimen (time: $\mathrm{F}_{(2,35)}=37.65, \mathrm{p}=0.0027$, Fig. $4 \mathrm{E}, 4 \mathrm{~F}, 4 \mathrm{G}, 4 \mathrm{H}$; number: $\mathrm{F}_{(2,35)}=8.04, \mathrm{p}=0.0013$, Supplementary Fig. 3b; one-way ANOVA). As expected, we observed significantly affected PEAP behaviour in vehicle-control versus naïves at week 7 post-surgery (time: $p=0.00019$, Fig. 4E, 4F, 4H; number: $\mathrm{p}=0.0010$, Supplementary Fig. 3b; Tukey-Kramer post hoc). Rats receiving delayed DHA treatment significantly improved cumulative time $(\sim 26 \%)$ and crossing number $(\sim 23 \%)$ compared to vehicle-control (time: $\mathrm{p}=0.0011$, Fig. 4F, 4G, 4H; number: $\mathrm{p}=0.0080$, Supplementary Fig. 3b; TukeyKramer post hoc), but their cumulative time was more than naïves ( $\mathrm{p}=0.030$, Tukey-Kramer post hoc). Pregabalin-controls in both studies showed comparable PEAP behaviour to DHA-treated rats (Table 2). 
These data indicate that systemic DHA treatment can prevent SCI-CNP at cognitive level shown by PEAP behavior.

\section{DHA significantly reduces microglial activation in vivo}

With immunohistochemistry, we revealed the effect of systemic DHA treatment on activated microglial morphology in L5 dorsal horns versus vehicle-control in the acute regimen study (p=0.0003, Fig. 5A(i), A(ii), A(iii), Mann-Whitney test). We next measured the expression of pp-38, a hallmark for SCI-CNP. Compared to vehicle-control, pp-38+/Iba-1+ microglial numbers in the dorsal horns were significantly reduced by DHA treatment in the acute regimen study [L5 (p=0.00019, Fig. 5B(i), B(ii), B(iii)); lesion site ( $p=0.00041$, Supplementary Fig. 9a-c); Mann-Whitney test]. Microgliosis, a key process in neuropathic pain pathogenesis, was significantly inhibited by DHA treatment versus vehicle as shown in the numbers of ED9+/CD11b/c+ microglia per mg cord tissue by flow cytometry [5] in the acute regimen study [L5 (p=0.048, Fig. 5C(i), C(ii), C(iii)); lesion site (p=0.038, Supplementary Fig. 9d-f); student $t$-test]. In the lesion site dorsal horns, we also found significant effect of the acute DHA regimen on activated microglial morphology ( $\mathrm{p}=0.00041$, Fig. 5D(i), D(ii), D(iii), Mann-Whitney test). We also saw a significant reduction in Iba1-immunoreactive microglial numbers in the ACC by the acute DHA regimen compared to vehicle-control (p=0.0006, Fig. 5E(i), E(ii), E(iii), Mann-Whitney test). In the delayed DHA regimen study, we found that in the dorsal horns activated microglial morphology [L5 (p=0.0005, Fig. 5F(i), F(ii), F(iii)); Mann-Whitney test] and pp-38+/Iba-1+ microglial numbers [L5 (p=0.00065, Fig. 5G(i), G(ii), G(iii)); lesion site ( $\mathrm{p}=0.00048$, Supplementary Fig. 9a-c); Mann-Whitney test] were significantly reduced by DHA treatment compared to vehicle-control. We also found significantly reduced numbers of ED9+/CD11b/c+ microglia per mg cord tissue [L5 (p=0.037, Fig. 5H(i), H(ii), H(iii)); lesion site ( $\mathrm{p}=0.048$, Supplementary Fig. $9 d-f)$; student $t$-test], activated microglial morphology in the lesion site dorsal horns ( $\mathrm{p}=0.00032$, Fig. 5I(i), I(ii), I(iii); Mann-Whitney test), and Iba1-immunoreactive microglial numbers in the ACC ( $\mathrm{p}=0.009$, Fig. 5J(i), J(ii), J(iii); Mann-Whitney test) by the delayed DHA 
regimen compared to vehicle-control. We noted that the proportion of pp-38+/Iba- $1+$ microglia in rats receiving DHA treatment in the acute regimen study was much lower than that seen in the delayed regimen study: $\sim 5 \%$ (Fig. 5Biii) vs 20\% (Fig. 5Giii). These data establish that systemic DHA treatment reduces spinal and supra-spinal microglial activation.

\section{DHA significantly spares white matter in vivo}

To assess the severity of the contusion SCI used in our studies and also to investigate the possible effects of systemic DHA treatments on tissue sparing that might underlie changes observed in pain-related behaviours, transverse cryostat sections from the lesion site segments were stained for myelin and white matter sparing was quantified at rostral, epicenter and caudal locations of the segments (Fig. 6A-6R). In the acute regimen study, large percentages of white matter were lost at the rostral, epicenter, and caudal sections of vehicle-treated animals when compared to naïve (Fig. 6A-6I). However, no difference in white matter sparing at three locations was observed between vehicle- and DHA-treated animals in the delayed regimen study (Fig. 6J-6O). Strikingly, systemic DHA treatments starting immediately following surgery significantly increased the percentages of white matter at three locations when compared to vehicle control ( $\mathrm{p}=0.0000015$ to 0.0000048 ; Fig. 6Q; Mann-Whitney test) at week 6 post-SCI.

\section{DHA significantly reduces proinflammatory mediators in vivo}

To further verify the effects of DHA treatment on proinflammatory and algogenic mediators iNOS, TNF$\alpha$, IL-1 $\beta$, and IL-6 in the spinal cord dorsal horns at lesion site and L5 levels, we carried out qPCR analysis of relative mRNA expression of these mediators. We found that at week 6 post-SCI, the acute DHA treatment regimen significantly reduced mRNA expression levels of iNOS, TNF- $\alpha$, IL-1 $\beta$, and IL6 at both lesion site and L5 dorsal horns compared to vehicle control ( $p=0.0002-0.032$; t-tests in R, Fig. 7A-7H). Moreover, we also found that delayed DHA treatment regimen starting week 4 post-SCI 
significantly reduced mRNA expression levels of these mediators at both dorsal horn levels at week 7 when compared to vehicle control ( $\mathrm{p}=0.004-0.048$, t-tests in R, Fig. 7I-7P).

\section{DHA significantly reduces microglial activation in vitro}

We also investigated the effects of DHA on modulating microglial activation in vitro. In experiments where cells were co-treated with activation agent Lipopolysaccharides (LPS) and DHA (0.8, 4, 8 or 32 $\mu \mathrm{M}$ ) for 4 hours, we found $0.8 \mu \mathrm{M}$ as the optimal concentration to reduce iNOS-immunoreactivity $\left(\mathrm{F}_{(5,50)}=76.15, \mathrm{p}=0.00024\right.$, one-way ANOVA, Supplementary Fig. 4a) (Fig. 8A(i), A(ii), A(iii), B(i), B(ii), B(iii)). With qPCR, we found that DHA significantly reduced microglial mRNA expression of algogenic mediators iNOS, TNF- $\alpha$, IL-1 $\beta$, and IL-6 compared to control $(\mathrm{p}=0.0091,0.0006,0.024$, and 0.026; $\mathrm{t}$ tests in R, Fig. 8C(i), C(ii), C(iii), C(iv)). Using the more sensitive flow cytometry, we confirmed the ability of DHA to reduce microglial activation, i.e. number of CD86+/CD11b/c+microglia $(\mathrm{p}=0.028$, student $t$ test; Fig. 8 D(i), D(ii), D(iii), D(iv)) versus control (LPS only). We also found significantly reduced numbers of iNOS+/Iba1+ microglia (Fig. 8E) and nitrite release by microglia (Fig. 8F) when cells were co-treated with LPS and DHA. Further experiments where cells were pre-activated by LPS for 4 hours and followed by DHA $(0.8 \mu \mathrm{M})$ treatment for another 4 hours showed similar effects on iNOS expression (Fig. 8G(i), G(ii), G(iii), H(i), H(ii), H(iii)), mRNA expression of iNOS, TNF- $\alpha$, IL-1 $\beta$, and IL-6 (Fig. 8I(i), I(ii), I(iii), I(iv)), and CD86+/CD11b/c+ number (Fig. 8 J(i), J(ii), J(iii), J(iv)), further validating the ability of DHA to modulate microglial activation.

\section{In vitro evidence of potential mechanisms of DHA on microglial activation}

Microglia express PPAR $\gamma$ and RXR receptors, which are known to involve in inflammation and can be bound by DHA [12-14]. Therefore, we used antagonists to pre-block PPAR $\gamma$ or RXR or both for 4 hours in cultured microglia, which were then co-treated with LPS and DHA $(0.8 \mu \mathrm{M})$ for 4 hours. With qPCR, 
we found that pre-antagonism of each receptor alone caused significantly higher mRNA expression of iNOS, TNF- $\alpha$, and IL-1 $\beta$ in microglia compared to without pre- antagonism (PPAR $\gamma: \mathrm{p}=0.003-0.0006$; RXR: p=0.00058-0.02; t-tests in R; Fig. 9A, 9B, 9C). When both receptors were pre-antagonised, we saw significantly higher microglial mRNA expression of iNOS, TNF- $\alpha$, and IL-6 ( $\mathrm{p}=0.0012-0.0020$; $\mathrm{t}-$ tests in R; Fig. 9A, 9B, 9D). These data suggest that DHA could act via these receptors to manifest its effects on reducing microglial activation. We finally investigated DHEA, a known DHA metabolite [19] that can bind to PPAR and cannabinoid receptors on microglia $[4 ; 6 ; 19 ; 41]$, by co-treating cultured microglia with LPS and DHEA for 4 hours. We saw a significant decrease in microglial mRNA expression of iNOS, TNF- $\alpha$, IL-1 $\beta$, and IL-6 by DHEA $(5 \mu \mathrm{M})$ when compared to control $(\mathrm{p}=0.0001$ 0.026; t-tests in R; Fig. 9E, 9F, 9G, 9H), suggesting that DHEA could partially mediate the effects of DHA on microglial activation. 
Discussion

SCI-CNP is a debilitating complication without effective treatments [21;52]. Novel strategies to effectively and safely manage SCI-CNP are needed urgently. Here we reported that systemic administrations of the natural compound DHA, starting immediately post-injury and then maintained for 6 weeks, successfully prevented the development of pain-related spontaneous/affective and cognitive behaviours coupled with significantly attenuated at-level mechanical hypersensitivity in a clinically relevant rat model of SCI-CNP. Similar DHA treatment, given at a chronic SCI stage when CNP had already been established, partially abolish pain-related spontaneous/affective and cognitive behaviours. Because our data were obtained using a novel sensory profiling approach combining evoked pain measures and non-evoked pain-related spontaneous and/or natural rodent behaviours to mimic the clinical practice, they have significant implications for clinical translation.

\section{DHA reduces neuroinflammation that underpins SCI-CNP pathogenesis}

Neuroinflammation plays a crucial role in SCI-CNP [25; 33]. Microglia become chronically activated in the lesion site and L5 dorsal horns as well as supraspinal areas [26; 28], subsequently activating ERK1/2, p-38 MAPK and CAMII pathways [25; 33] that are known to be important for SCI-CNP development. The activation of p-38 MAPK, a hallmark for SCI-CNP [10; 17], causes the release of proinflammatory mediators, including iNOS, TNF- $\alpha$, IL-1 $\beta$, IL-6, PGE2, and cysteine chemokine ligand 21, producing neuronal hyperexcitability $[17 ; 25 ; 44 ; 60-62]$. Moreover, astrocytes are also documented for their contribution to SCI-CNP [25; 33]. Here, we showed that DHA in both regimen studies significantly decreased activated microglial/astrocyte morphologies, microgliosis, and GFAP immunoreactivity in lesion site/L5 dorsal horns. DHA significantly reduced microglial pp-38 MAPK expression in lesion site/ L5 dorsal horns, with the acute regimen having 4-fold more reduction than the delayed regimen. We also 
observed significantly reduced microglial/astrocyte numbers in the ACC of DHA-treated animals from both studies. Furthermore, mRNA levels of proinflammatory mediators in lesion site/L5 dorsal horns were significantly reduced by DHA treatments. DHA also reduced mRNA levels of proinflammatory mediators in LPS-activated microglia in vitro. Thus, the effects on CNP prevention in the acute regimen study and on ameliorating CNP partially in the delayed regimen study are likely due to significantly attenuated microglial/astrocyte activation at both spinal and supra-spinal levels.

\section{DHA's effects on dorsal horn neuronal hyperexcitability}

Electrophysiological studies show that SCI results in neuronal hyperexcitability in the dorsal horns at and below the injury level, which mediates enhanced nociceptive processing in pathological pain states [10; 27; 28]. Particularly, pp-38 MAPK plays a pivotal role underlying dorsal horn neuronal hyperexcitability as discussed above. Thus, increased microglial pp-38 MARK levels corelate with dorsal horn neuronal hyperresponsiveness and CNP following contusion SCI in adult rats [10; 28]. Moreover, minocycline and pp-38 inhibitor SB203580 significantly reduce dorsal horn microglial pp-38 MAPK, which results in attenuated dorsal horn neuronal hyperexcitability. The downstream pathways activated by microglial pp-38 MAPK lead to the release of numerous proinflammatory mediators that can bind to and activate dorsal horn neuronal membrane receptors and/or ion channels, triggering neuronal pp-38 MAPK-CREB-ERK pathway that causes persistent neuronal hyperexcitability [25]. Among those mediators, TNF- $\alpha$, IL-1 $\beta$ and IL-6 produced by microglia after SCI are known to produce hyperactivity of dorsal horn neurons and contribute to CNP development and maintenance $[17 ; 40]$. Our results demonstrated that DHA significantly reduced microglial pp-38 MAPK immunoreactivity in lesion site/L5 dorsal horns, which is mirrored by a significant reduction of mRNA levels of iNOS, TNF- $\alpha$, IL$1 \beta$ and IL-6 in lesion site/L5 dorsal horns. These data strongly suggest that DHA might have stopped the positive feedforward cycle between microglia and dorsal horn neurons that maintains persistent neuronal 
hyperexcitability, leading to the prevention of CNP seen in the acute study and partial reversal of CNP seen in the delayed study. Here, we did not assess neuronal/astrocyte pp-38 MAPK expressions, which also contribute to dorsal horn neuronal hyperexcitability after SCI [10; 25]. Because DHA could interact with receptors and ion channels on dorsal horn neurons and astrocytes as discussed below, we suggest that DHA treatments may have also reduced neuronal/astrocyte pp-38 MAPK levels in lesion site/L5 dorsal horns.

\section{Mechanisms of DHA's action in reducing neuroinflammation}

Recent evidence shows that dietary DHA supplementation for 60 days leads to increased DHA levels in retina, cortex, hypothalamus and hippocampus in adult rats [16]. Other evidence shows the incorporation of intravenously injected radiolabeled DHA into the rat brain [45]. Therefore, we are confident that DHA in our studies would have reached the spinal cord of SCI rats. DHA's effects on attenuating microglial/astrocyte activation are likely mediated by DHA binding to and activating PPAR and RXR receptors as their natural ligand [12-14], since these receptors are expressed by microglia [38; 51] and astrocytes $[18 ; 51]$. This is supported by our evidence demonstrating that pre-inhibiting these receptors reduced DHA's ability to decrease mRNA levels of pain-producing proinflammatory mediators in cultured microglia co-exposed to LPS. It is possible that DHA's effects on microglial activation could be via its metabolite DHEA [19], an endocannabinoid, which can bind to PPAR and cannabinoid receptors $[4 ; 6 ; 19 ; 41]$ on microglia. This notion is supported by our data showing that DHEA significantly reduced mRNA levels of proinflammatory mediators in cultured microglia co-exposed to LPS. Future studies are needed to measure DHA/DHEA levels in the spinal cord and explore the full role of DHEA on microglial activation which is beyond the scope of current studies.

\section{DHA’s unique potential to target multiple mechanisms underlying SCI-CNP}


We suggest that DHA's effects on SCI-CNP could be attributed to its neuroprotection of neurons and oligodendrocytes [32], which was supported by improved locomotor function in both regimen studies and increased white matter sparing in the acute regimen study. Thus, DHA may interact with glutamate receptors [32] and voltage-gated sodium/calcium channels [55] on dorsal horn neurons, which are known to play roles in neuronal hyperexcitability. This makes DHA a promising treatment for SCI-CNP, as it can target multiple mechanisms underlying SCI-CNP.

\section{A novel sensory profiling approach mimics clinical practice}

Our work is timely in addressing a recent call for the use of sensory profiling in animal models [46]. The preclinical literature on SCI-CNP mainly relies on spinal reflex-based withdrawal paradigms, which hardly reflect the affective, cognitive and spontaneous aspects of pain and have minimal clinical validity. Our previous and current work showed that below-level SCI-CNP in rats, measured by mechanically evoked hindpaw withdrawal thresholds, did not involve brainstem responses, which is likely due to the presence of spastic syndrome [3]. Here we combined at-level mechanical hypersensitivity basing on brainstem responses with pain-related spontaneous and/or natural rodent behaviours addressing the motivational, affective and cognitive components of pain. These pain-related behaviours included burrowing, thigmotaxis, and PEAP, which are ethologically relevant and reproducible in different laboratory settings. Most importantly, they mimic those used for measuring the impact of pain and spontaneous component of pain in clinical settings. Such an approach would significantly add translational benefit for new drug development for SCI-CNP.

Consistent with our previous reports $[2 ; 3]$, we observed that all rats with the injury severity adopted here developed brainstem response-based at-level mechanical hypersensitivity. Therefore, our SCI-CNP model does not reflect SCI-CNP prevalence seen in human patients. It is known that different injury severities and injury devices used can influence SCI-CNP prevalence in animals [33]. Similar situations 
also exist in common preclinical models of peripheral nerve trauma-induced neuropathic pain and druginduced peripheral neuropathies, all of which are instrumental in understanding the pathophysiological mechanisms and developing novel analgesics for neuropathic pain.

\section{DHA's translational potential for SCI-CNP management}

We suggest that the two DHA regimens could be readily translated to the clinic, because DHA's clinical safety and tolerability is well documented. DHA-containing fish oil capsules are available for public consumption. Other DHA-containing preparations are already used in clinical settings involving surgical and critically ill patients [8]. A recent 14-month trial with consumption of DHA-containing capsules in chronic SCI patients did not find neuroprotective effects, but proved DHA's long-term patient safety [34]. In our studies, burrowing behaviour, which is conserved in rodents and reflects their general wellbeing, was significantly improved in DHA-treated rats, further demonstrating the safety and translational potential of DHA for SCI-CNP management in the clinic. We suggest that future studies should investigate the pharmacokinetics of DHA, the optimal dose, best administration route (e.g. i.v. vs dietary supplementation) and formulation (e.g. free fatty acid format used here $v s$ lipid emulsions) for systemic DHA treatment for SCI-CNP and to establish if the effects on SCI-CNP by the two DHA regimens would continue if treatments stop, which are beyond the current scope. Further discussion on DHA's preclinical translation addressing pharmacokinetics and relevance of concentrations to target pharmacology can be found in Supplementary Discussion Section.

In summary, our studies using clinically relevant animal modelling, revealed huge potential of systemic DHA treatments for not only preventing SCI-CNP but remarkably also for treating already established SCI-CNP. Considering the unmet clinical need for SCI-CNP, our strategies involving systemic DHA treatments represent one of the most promising approaches available for SCI-CNP management. These 
strategies also could benefit millions of patients who suffer from CNP following other CNS injuries and diseases including brain trauma, stroke, and multiple sclerosis.

Acknowledgments

We thank Ms. Leilani Barry and Ms. Hristina Stefanova for their assistance with immunocytochemistry and qPCR work. We thank Dr Franziska Denk for her kind advice on in vivo tissue qPCR. We are grateful of Prof Colin McCaig for his critical and constructive review of the manuscript. We thank the Development Trust of The University of Aberdeen and the Scottish Rugby Union for their financial support. Part of the revision work was supported by a Science Initiatives Panel Award by the Institute of Medical Sciences of the University of Aberdeen.

A conflict of interest statement: N.B.F. has received honoraria for serving on advisory boards or speaker panels from Teva, Novartis, Astellas, Grünenthal, Mitshubishe Tanabe, Novartis and Teva Pharmaceuticals outside the submitted work. All authors declare no competing financial interests. 


\section{References}

[1] Andrews N, Legg E, Lisak D, Issop Y, Richardson D, Harper S, Pheby T, Huang W, Burgess G, Machin I, Rice AS. Spontaneous burrowing behaviour in the rat is reduced by peripheral nerve injury or inflammation associated pain. Eur J Pain 2012;16(4):485-495.

[2] Baastrup C, Jensen TS, Finnerup NB. Pregabalin attenuates place escape/avoidance behavior in a rat model of spinal cord injury. Brain Res 2011;1370:129-135.

[3] Baastrup C, Maersk-Moller CC, Nyengaard JR, Jensen TS, Finnerup NB. Spinal-, brainstem- and cerebrally mediated responses at- and below-level of a spinal cord contusion in rats: evaluation of pain-like behavior. Pain 2010;151(3):670-679.

[4] Balvers MG, Verhoeckx KC, Plastina P, Wortelboer HM, Meijerink J, Witkamp RF. Docosahexaenoic acid and eicosapentaenoic acid are converted by 3T3-L1 adipocytes to N-acyl ethanolamines with anti-inflammatory properties. Biochim Biophys Acta 2010;1801(10):11071114.

[5] Blackbeard J, Wallace VC, O'Dea KP, Hasnie F, Segerdahl A, Pheby T, Field MJ, Takata M, Rice AS. The correlation between pain-related behaviour and spinal microgliosis in four distinct models of peripheral neuropathy. Eur J Pain 2012;16(10):1357-1367.

[6] Brown I, Cascio MG, Wahle KW, Smoum R, Mechoulam R, Ross RA, Pertwee RG, Heys SD. Cannabinoid receptor-dependent and -independent anti-proliferative effects of omega-3 ethanolamides in androgen receptor-positive and -negative prostate cancer cell lines. Carcinogenesis 2010;31(9):1584-1591.

[7] Burke D, Fullen BM, Stokes D, Lennon O. Neuropathic pain prevalence following spinal cord injury: A systematic review and meta-analysis. Eur J Pain 2017;21(1):29-44.

[8] Calder PC. Long-chain n-3 fatty acids and inflammation: potential application in surgical and trauma patients. Braz J Med Biol Res 2003;36(4):433-446. 
[9] Chaplan SR, Bach FW, Pogrel JW, Chung JM, Yaksh TL. Quantitative assessment of tactile allodynia in the rat paw. J Neurosci Methods 1994;53(1):55-63.

[10] Crown ED, Gwak YS, Ye Z, Johnson KM, Hulsebosch CE. Activation of p38 MAP kinase is involved in central neuropathic pain following spinal cord injury. Exp Neurol 2008;213(2):257267.

[11] Cryan JF, Holmes A. The ascent of mouse: advances in modelling human depression and anxiety. Nat Rev Drug Discov 2005;4(9):775-790.

[12] Dawson MI, Xia Z. The retinoid X receptors and their ligands. Biochim Biophys Acta 2012;1821(1):21-56.

[13] Daynes RA, Jones DC. Emerging roles of PPARS in inflammation and immunity. Nat Rev Immunol 2002;2(10):748-759.

[14] de Urquiza AM, Liu S, Sjoberg M, Zetterstrom RH, Griffiths W, Sjovall J, Perlmann T. Docosahexaenoic acid, a ligand for the retinoid $\mathrm{X}$ receptor in mouse brain. Science 2000;290(5499):2140-2144.

[15] Denk F, Huang W, Sidders B, Bithell A, Crow M, Grist J, Sharma S, Ziemek D, Rice AS, Buckley NJ, McMahon SB. HDAC inhibitors attenuate the development of hypersensitivity in models of neuropathic pain. Pain 2013;154(9):1668-1679.

[16] Destaillats F, Oliveira M, Bastic Schmid V, Masserey-Elmelegy I, Giuffrida F, Thakkar SK, Dupuis L, Gosoniu ML, Cruz-Hernandez C. Comparison of the Incorporation of DHA in Circulatory and Neural Tissue When Provided as Triacylglycerol (TAG), Monoacylglycerol (MAG) or Phospholipids (PL) Provides New Insight into Fatty Acid Bioavailability. Nutrients 2018;10(5).

[17] Detloff MR, Fisher LC, McGaughy V, Longbrake EE, Popovich PG, Basso DM. Remote activation of microglia and pro-inflammatory cytokines predict the onset and severity of below-level neuropathic pain after spinal cord injury in rats. Exp Neurol 2008;212(2):337-347. 
[18] Fandel D, Wasmuht D, Avila-Martin G, Taylor AJ, Galan-Arriero I, Mey J. Spinal cord injury induced changes of nuclear receptors PPAR $\alpha$ and LXR $\beta$ and modulation with oleic acid/albumin treatment. Brain Res 2013;1535:89-105.

[19] Figueroa JD, Cordero K, Serrano-Illan M, Almeyda A, Baldeosingh K, Almaguel FG, De Leon M. Metabolomics uncovers dietary omega-3 fatty acid-derived metabolites implicated in antinociceptive responses after experimental spinal cord injury. Neuroscience 2013;255:1-18.

[20] Finnerup N, Baastrup C. Spinal Cord Injury Pain: Mechanisms and Management. Current Pain and Headache Reports 2012;16(3):207-216.

[21] Finnerup NB, Jensen MP, Norrbrink C, Trok K, Johannesen IL, Jensen TS, Werhagen L. A prospective study of pain and psychological functioning following traumatic spinal cord injury. Spinal Cord 2016;54(10):816-821.

[22] Georgieva M, Leeson-Payne A, Dumitrascuta M, Rajnicek A, Malcangio M, Huang W. A refined rat primary neonatal microglial culture method that reduces time, cost and animal use. J Neurosci Methods 2018.

[23] German OL, Monaco S, Agnolazza DL, Rotstein NP, Politi LE. Retinoid X receptor activation is essential for docosahexaenoic acid protection of retina photoreceptors. J Lipid Res 2013;54(8):2236-2246.

[24] Grygiel-Gorniak B. Peroxisome proliferator-activated receptors and their ligands: nutritional and clinical implications--a review. Nutr J 2014;13:17.

[25] Gwak YS, Hulsebosch CE, Leem JW. Neuronal-Glial Interactions Maintain Chronic Neuropathic Pain after Spinal Cord Injury. Neural plasticity 2017;2017:2480689.

[26] Gwak YS, Kang J, Unabia GC, Hulsebosch CE. Spatial and temporal activation of spinal glial cells: role of gliopathy in central neuropathic pain following spinal cord injury in rats. Exp Neurol $2012 ; 234(2): 362-372$. 
[27] Hains BC, Johnson KM, Eaton MJ, Willis WD, Hulsebosch CE. Serotonergic neural precursor cell grafts attenuate bilateral hyperexcitability of dorsal horn neurons after spinal hemisection in rat. Neuroscience 2003;116(4):1097-1110.

[28] Hains BC, Waxman SG. Activated microglia contribute to the maintenance of chronic pain after spinal cord injury. J Neurosci 2006;26(16):4308-4317.

[29] Hong C, Tontonoz P. Coordination of inflammation and metabolism by PPAR and LXR nuclear receptors. Curr Opin Genet Dev 2008;18(5):461-467.

[30] Huang W, Calvo M, Karu K, Olausen HR, Bathgate G, Okuse K, Bennett DL, Rice AS. A clinically relevant rodent model of the HIV antiretroviral drug stavudine induced painful peripheral neuropathy. Pain 2013;154(4):560-575.

[31] Huang W, Calvo M, Pheby T, Bennett DL, Rice AS. A rodent model of HIV protease inhibitor indinavir induced peripheral neuropathy. Pain 2017;158(1):75-85.

[32] Huang WL, King VR, Curran OE, Dyall SC, Ward RE, Lal N, Priestley JV, Michael-Titus AT. A combination of intravenous and dietary docosahexaenoic acid significantly improves outcome after spinal cord injury. Brain 2007;130(Pt 11):3004-3019.

[33] Hulsebosch CE, Hains BC, Crown ED, Carlton SM. Mechanisms of chronic central neuropathic pain after spinal cord injury. Brain Res Rev 2009;60(1):202-213.

[34] Javidan AN, Sabour H, Latifi S, Abrishamkar M, Soltani Z, Shidfar F, Razavi HE. Does consumption of polyunsaturated fatty acids influence on neurorehabilitation in traumatic spinal cord-injured individuals? a double-blinded clinical trial. Spinal Cord 2014;52:378-382.

[35] Kimura R, Takahashi N, Lin S, Goto T, Murota K, Nakata R, Inoue H, Kawada T. DHA attenuates postprandial hyperlipidemia via activating PPARalpha in intestinal epithelial cells. J Lipid Res 2013;54(12):3258-3268.

[36] Lim SN, Huang W, Hall JC, Michael-Titus AT, Priestley JV. Improved outcome after spinal cord compression injury in mice treated with docosahexaenoic acid. Exp Neurol 2013;239:13-27. 
[37] Lu DY, Tsao YY, Leung YM, Su KP. Docosahexaenoic acid suppresses neuroinflammatory responses and induces heme oxygenase-1 expression in BV-2 microglia: implications of antidepressant effects for omega-3 fatty acids. Neuropsychopharmacology 2010;35(11):22382248.

[38] Lue LF, Kuo YM, Beach T, Walker D. Microglia Activation and Anti-inflammatory Regulation in Alzheimer's Disease. Mol Neurobiol 2010;41(2):115-128.

[39] Macleod MR, Fisher M, O'Collins V, Sena ES, Dirnagl U, Bath PM, Buchan A, van der Worp HB, Traystman R, Minematsu K, Donnan GA, Howells DW. Good laboratory practice: preventing introduction of bias at the bench. Stroke 2009;40(3):e50-52.

[40] Marchand F, Tsantoulas C, Singh D, Grist J, Clark AK, Bradbury EJ, McMahon SB. Effects of Etanercept and Minocycline in a rat model of spinal cord injury. Eur J Pain 2009;13(7):673-681.

[41] McDougle DR, Watson JE, Abdeen AA, Adili R, Caputo MP, Krapf JE, Johnson RW, Kilian KA, Holinstat M, Das A. Anti-inflammatory omega-3 endocannabinoid epoxides. Proc Natl Acad Sci U S A 2017;114(30):E6034-E6043.

[42] Morland RH, Novejarque A, Huang W, Wodarski R, Denk F, Dawes JD, Pheby T, McMahon SB, Rice AS. Short-term effect of acute and repeated urinary bladder inflammation on thigmotactic behaviour in the laboratory rat. F1000Research 2015;4:109.

[43] Moss PJ, Huang W, Dawes J, Okuse K, McMahon SB, Rice AS. Macrophage-sensory neuronal interaction in HIV-1 gp120-induced neurotoxicitydouble dagger. British journal of anaesthesia 2015;114(3):499-508.

[44] Peng XM, Zhou ZG, Glorioso JC, Fink DJ, Mata M. Tumor necrosis factor-alpha contributes to below-level neuropathic pain after spinal cord injury. Ann Neurol 2006;59(5):843-851.

[45] Rapoport SI, Ramadan E, Basselin M. Docosahexaenoic acid (DHA) incorporation into the brain from plasma, as an in vivo biomarker of brain DHA metabolism and neurotransmission. Prostaglandins Other Lipid Mediat 2011;96(1-4):109-113. 
[46] Rice ASC, Finnerup NB, Kemp HI, Currie GL, Baron R. Sensory profiling in animal models of neuropathic pain: a call for back-translation. Pain 2018;159(5):819-824.

[47] Rice ASC, Morland R, Huang W, Currie GL, Sena ES, MaCleod MR. Transparency in the reporting of in vivo pre-clinical pain research: The relevance and implications of the ARRIVE (Animal Research: Reporting In Vivo Experiments) guideline. Scan J Pain 2013;4(58-62).

[48] Robson LG, Dyall S, Sidloff D, Michael-Titus AT. Omega-3 polyunsaturated fatty acids increase the neurite outgrowth of rat sensory neurones throughout development and in aged animals. Neurobiol Aging 2010;31(4):678-687.

[49] Rutten K, Schiene K, Robens A, Leipelt A, Pasqualon T, Read SJ, Christoph T. Burrowing as a nonreflex behavioural readout for analgesic action in a rat model of sub-chronic knee joint inflammation. Eur J Pain 2014;18(2):204-212.

[50] Schmittgen TD, Livak KJ. Analyzing real-time PCR data by the comparative C(T) method. Nat Protoc 2008;3(6):1101-1108.

[51] Schrage K, Koopmans G, Joosten EAJ, Mey J. Macrophages and neurons are targets of retinoic acid signaling after spinal cord contusion injury. Eur J Neurosci 2006;23(2):285-295.

[52] Siddall PJ, McClelland JM, Rutkowski SB, Cousins MJ. A longitudinal study of the prevalence and characteristics of pain in the first 5 years following spinal cord injury. Pain 2003;103(3):249-257.

[53] Sliwinski C, Nees TA, Puttagunta R, Weidner N, Blesch A. Sensorimotor Activity Partially Ameliorates Pain and Reduces Nociceptive Fiber Density in the Chronically Injured Spinal Cord. Journal of neurotrauma 2018;35(18):2222-2238.

[54] Varga T, Czimmerer Z, Nagy L. PPARs are a unique set of fatty acid regulated transcription factors controlling both lipid metabolism and inflammation. Biochim Biophys Acta 2011;1812(8):10071022. 
[55] Vreugdenhil M, Bruehl C, Voskuyl RA, Kang JX, Leaf A, Wadman WJ. Polyunsaturated fatty acids modulate sodium and calcium currents in CA1 neurons. Proc Natl Acad Sci U S A 1996;93(22):12559-12563.

[56] Wodarski R, Delaney A, Ultenius C, Morland R, Andrews N, Baastrup C, Bryden LA, Caspani O, Christoph T, Gardiner NJ, Huang W, Kennedy JD, Koyama S, Li D, Ligocki M, Lindsten A, Machin I, Pekcec A, Robens A, Rotariu SM, Vo S, Segerdahl M, Stenfors C, Svensson CI, Treede RD, Uto K, Yamamoto K, Rutten K, Rice AS. Cross-centre replication of suppressed burrowing behaviour as an ethologically relevant pain outcome measure in the rat: a prospective multicentre study. Pain 2016;157(10):2350-2365.

[57] Wollaars MM, Post MWM, van Asbeck FWA, Brand N. Spinal Cord Injury Pain: The Influence of Psychologic Factors and Impact on Quality of Life. Clin J Pain 2007;23(5):383-391.

[58] Yamamoto A, Kakuta H, Miyachi H, Sugimoto Y. Involvement of the Retinoid X Receptor Ligand in the Anti-Inflammatory Effect Induced by Peroxisome Proliferator-Activated Receptor gamma Agonist In Vivo. PPAR Res 2011;2011:840194.

[59] Zapata-Gonzalez F, Rueda F, Petriz J, Domingo P, Villarroya F, Diaz-Delfin J, de Madariaga MA, Domingo JC. Human dendritic cell activities are modulated by the omega-3 fatty acid, docosahexaenoic acid, mainly through PPAR(gamma):RXR heterodimers: comparison with other polyunsaturated fatty acids. J Leukoc Biol 2008;84(4):1172-1182.

[60] Zhao P, Waxman SG, Hains BC. Extracellular signal-regulated kinase-regulated microglia-neuron signaling by prostaglandin E2 contributes to pain after spinal cord injury. J Neurosci 2007;27(9):2357-2368.

[61] Zhao P, Waxman SG, Hains BC. Modulation of thalamic nociceptive processing after spinal cord injury through remote activation of thalamic microglia by cysteine cysteine chemokine ligand 21 . J Neurosci 2007;27(33):8893-8902. 
[62] Zhou YQ, Liu Z, Liu ZH, Chen SP, Li M, Shahveranov A, Ye DW, Tian YK. Interleukin-6: an emerging regulator of pathological pain. J Neuroinflammation 2016;13(1):141.

[63] Zuniga J, Cancino M, Medina F, Varela P, Vargas R, Tapia G, Videla LA, Fernandez V. N-3 PUFA supplementation triggers PPAR-alpha activation and PPAR-alpha/NF-kappaB interaction: antiinflammatory implications in liver ischemia-reperfusion injury. PLoS One 2011;6(12):e28502. 
Figure legends

Fig. 1: Effects of systemic DHA treatments on below- and at-level mechanical hypersensitivity. (A, B): Study designs of acute and delayed DHA treatment regimens in vivo. DHA administered every 3 days, starting either 30 minutes $(\mathbf{C})$ or week 4 (D) post-SCI, significantly increased hindpaw withdrawal thresholds to punctate mechanical stimuli, comparing to those of vehicle-treated rats (C: weeks 2-6, $\mathrm{p}<0.01$ and $\mathbf{D}$ : weeks 5-7, $<<0.001)$. Both the acute and the delayed regimens significantly increased brainstem response thresholds to at-level mechanical stimulations, comparing to those of vehicle-treated rats $(\mathbf{E}$ : weeks 2-6, p<0.05; F: weeks 5-7, p<0.05). No differences in brainstem responses to hindpaw mechanical stimulations were found in both regimen studies $(\mathbf{G}, \mathbf{H})$. N=10-15 per group. Two-way repeated-measures ANOVA was used to determine the main effects of treatment (DHA vs vehicle) and time (weekly). Statistical significance of the differences between the groups was determined by one-way ANOVA followed by Tukey-Kramer post hoc test at each week point. $*_{\mathrm{p}}<0.05$, **p<0.01, or $* * * \mathrm{p}<0.001$ vs vehicle.

Fig. 2: Effects of systemic DHA treatments on burrowing behaviour. In the acute regimen study, DHA-treated rats had significantly increased burrowing performances in contrast to vehicle-treated rats (A: weeks 2-6, p<0.01). Moreover, there were no significant differences in burrowing performances between DHA-treated and naïve rats (A: weeks 2-6, p>0.05). In the delayed regimen study, burrowing performances of SCI rats receiving DHA treatment were significantly attenuated in comparison to those observed in vehicle-treated rats $(\mathbf{B}$ : weeks 5-7, p<0.05), but still significantly lower than those of the naïve rats (B: weeks 4-7, p<0.05). N=10-15 per group. Two-way repeated-measures ANOVA was used to determine the main effects of treatment (DHA vs vehicle) and time (weekly). Statistical significance of the differences between the groups was determined by one-way ANOVA followed by Tukey-Kramer post hoc test at each week point. ${ }^{*} \mathrm{p}<0.05, * * \mathrm{p}<0.01$, or $* * * \mathrm{p}<0.001$ vs vehicle. 
Fig. 3: Effects of systemic DHA treatments on thigmotactic behaviour. (A-C, E-G): representative spontaneous movement tracks recorded for the naïve, vehicle-treated, and DHA-treated rats at weeks 6 and 7 post-surgery for the acute and delayed regimen studies. (D): analysis showed that the acute regimen significantly increased inner zone time, comparing to that of vehicle treatment $(* * \mathrm{p}<0.01$, Tukey-Kramer post hoc). There was no significant difference in inner zone time between DHA-treated and naïve rats $(\mathrm{p}>0.05)$. $(\mathbf{H})$ : analysis showed that the delayed regimen also significantly increased inner zone time, in contrast to vehicle treatment $(* \mathrm{p}<0.05)$; but the time was still significantly less than that of the naïve rats $(* \mathrm{p}<0.05) . \mathrm{N}=10-15$ per group. One-way ANOVA followed by Tukey-Kramer post hoc test were used. White dotted square boxes=open field arenas. Yellow and white lines=the widths of the open field arena and virtual inner zone.

Fig. 4: Effect of systemic DHA treatments on PEAP behaviour. (A-C, E-G): Representative free movement tracks recorded for naïve, vehicle-treated, and DHA-treated rats at weeks 6 and 7 post-surgery for the two regimen studies. (D): analysis showed that the acute regimen led to a significant reduction in white zone cumulative time comparing to vehicle treatment $(* * p<0.01)$. Markedly, there was no difference in white zone cumulative time between DHA-treated and naïve rats ( $>>0.05)$. (H): analysis showed that the delayed regimen also significantly decreased white zone cumulative time contrasting to vehicle treatment $(* * \mathrm{p}<0.01)$; but the time was still significantly more than that of the naïve rats $\left({ }^{*} \mathrm{p}<0.05\right) . \mathrm{N}=10-15$ per treatment group. One-way ANOVA followed by Tukey-Kramer post hoc test were used for statistical analysis.

Fig. 5: Effects of systemic DHA treatments on microglial activation in vivo. Both regimen studies showed significantly reduced activated microglial morphology per $50,000 \mu \mathrm{m}^{2}$ by DHA treatments in L5 (A(i-iii) and $\left.\mathbf{F}_{(\text {i-iii) }}\right)$ and lesion site $\left(\mathbf{D}_{(\text {i-iii) }}\right.$ and $\left.\mathbf{I}_{(\text {i-iii) }}\right)$ dorsal horns (DH), comparing to vehicle treatments. Both DHA regimens led to significant reductions of microglial pp38 immunoreactivity in L5 dorsal 
horns, comparing to vehicle treatments $\left(\mathbf{B}_{\text {(i-iii) }}\right.$ and $\mathbf{G}_{\text {(i-iii); }}$ white arrows=Iba-1/pp38 doubleimmunoreactive microglia). Flow cytometry further showed that both DHA regimens $\left(\mathbf{C}_{(\mathrm{i} \text {-iii) }}\right.$ and $\left.\mathbf{H}_{(\mathrm{i} \text {-iii })}\right)$ significantly reduced microgliosis, i.e. the numbers of proinflammatory CD11b/c+/ED9+ microglia in L5 spinal cords, comparing to vehicle treatments; $\mathbf{C}_{(\mathbf{i}-\mathrm{ii})}$ and $\mathbf{H}_{(\mathrm{i}-\mathrm{ii})}=$ examples of flow cytometry data from vehicle- and DHA-treated rats. Finally, both DHA regimens $\left(\mathbf{E}_{(\mathbf{i} \text {-iii) }}\right.$ and $\left.\mathbf{J}_{(\mathbf{i}-i i i)}\right)$ led to marked reductions in Iba-1 immunoreactive microglia in the ACC, in contrast to vehicle treatments. N=5-6 per group. Mann-Whitney test and unpaired student's $t$ test were used where appropriate. $(\mathrm{K})$ is a schematic drawing of the lesion site segment from where lesion site DHs were analyzed for microglial Iba-1 staining. ${ }^{*} \mathrm{p}<0.05, * * \mathrm{p}<0.01, * * * \mathrm{p}<0.001$ vs vehicle.

Fig. 6: Effects of systemic DHA treatments on tissue sparing. Representative euriochrome cyaninestained transverse sections are shown for naïve rats (A-C), SCI rats with Vehicle (D-F) or DHA (G-I) treatments in the acute regimen study, and SCI rats with Vehicle (J-L) and DHA (M-O) treatments in the delayed regimen study. (P) is a schematic drawing of the lesion site segment, from where rostral, epicenter, and caudal transverse sections were chosen for the staining. The lesion site epicenter showed large cavity formation accompanied by the loss of gray and white matter in vehicle-treated animals and SCI rats received delayed DHA treatment. (Q-R) Quantification of the cross-sectional area (CSA) indicates significant losses of white matter in vehicle control groups at three locations analyzed in both studies. In contrast, SCI rats received the acute DHA treatment regimen had significantly more spared myelin (****p<0.0001 vs vehicle-treated animals; Q). N=4-5 per group. Mann-Whitney test was used for statistical analysis. Scale bar $=1 \mathrm{~mm}$.

Fig. 7: Effects of systemic DHA treatments on the production of proinflammatory mediators in the dorsal horns of lesion site and L5 spinal cords. qPCR analysis showed that iNOS, TNF- $\alpha$, IL-1 $\beta$ and IL-6 mRNA expression levels were significantly reduced at week 6 following the acute DHA 
treatment regimen $(\mathrm{A}-\mathrm{H})$ and at week 7 following the delayed DHA treatment regimen (I-P), when compared to the vehicle groups. The relative mRNA expression levels of these proinflammatory mediators were similar between DHA-treated and naïve animals. N=4-5 per group. Statistical analysis was performed by $\mathrm{t}$-tests in $\mathrm{R} .{ }^{*} \mathrm{p}<0.05, * * \mathrm{p}<0.01, * * * \mathrm{p}<0.001 v s$ vehicle-treated animals.

Fig. 8: DHA modulates microglial activation in vitro. Microglia, simultaneously treated (ST) with DHA and LPS, preserved resting bipolar morphology (A(iii) $)$ seen with the controls $\left(\mathbf{A}_{(\mathbf{i})}\right)$, contrasting to activated morphology seen with LPS only (A(ii)). DHA in ST experiments reduced iNOS expression, mRNA expression levels of iNOS, IL-6, TNF- $\alpha$ and IL-1 $\beta$, and nitrite releases by microglia, comparing to LPS only (B(ii-iii), $\left.\mathbf{C}_{(\mathbf{i}-\mathrm{iv})}, \mathbf{E}-\mathbf{F}\right)$. Microglia with delayed DHA treatment (DT) after 4 hours of LPS preexposure $\left(\mathbf{G}_{(i i i)}\right)$ reversed activated morphology seen with LPS only $\left(\mathbf{G}_{(\mathrm{ii})}\right)$. DHA in DT experiments also reduced iNOS expression, mRNA expression levels of iNOS, IL-6, TNF- $\alpha$ and IL-1 $\beta$, and nitrite releases by microglia, comparing to LPS only $\left.\left(\mathbf{H}_{(\mathrm{ii}-\mathrm{iii})}, \mathbf{I}_{(\mathbf{i}-\mathrm{i})}\right), \mathbf{K}-\mathbf{L}\right)$. Flow cytometry further verified that the numbers of proinflammatory CD11b/c+/CD86+ microglia were significantly reduced by DHA in ST and DT experiments, comparing to LPS only $\left(\mathbf{D}_{(\mathbf{i}-\mathbf{i v})}, \mathbf{J}_{(\mathbf{i}-\mathbf{i v})}\right) . \mathrm{N}=3$ biological replicates/n=9 technical replicates per group. Unpaired student's $t$-test and t-tests in $\mathrm{R}$ were used where appropriate. ${ }^{*} \mathrm{p}<0.05$, $* * \mathrm{p}<0.01, * * * \mathrm{p}<0.001$ vs LPS-treated microglia.

Fig. 9: DHA's mechanisms on modulating microglial activation in vitro. Pre-treating cultured microglia with PPAR/RXR receptor antagonists, alone or both, reduced DHA's effects on decreasing mRNA expression levels of TNF- $\alpha$ (A), iNOS (B), IL-1 $\beta$ (C) and IL-6 (D), in contrast to without PPAR/RXR receptor pre-blockage. Treating cultured LPS-exposed microglia with DHEA significantly reduced mRNA expression levels of TNF- $\alpha(\mathbf{E})$, iNOS $(\mathbf{F})$, IL-1 $\beta(\mathbf{G})$ and IL-6 $(\mathbf{H})$, comparing to LPS 
only. $\mathrm{N}=5$ biological replicates per group. Statistical analysis was performed by t-tests in $\mathrm{R} .{ }^{*} \mathrm{p}<0.05$, $* * \mathrm{p}<0.01, * * * \mathrm{p}<0.001$ vs DHA-treated (A-D) and $v s$ LPS-treated $(\mathbf{E}-\mathbf{H})$. 
1

\section{Summary}

Systemic treatments with docosahexaenoic acid prevent central pain development following spinal injury and partially abolish already established central pain at a chronic stage of injury. 

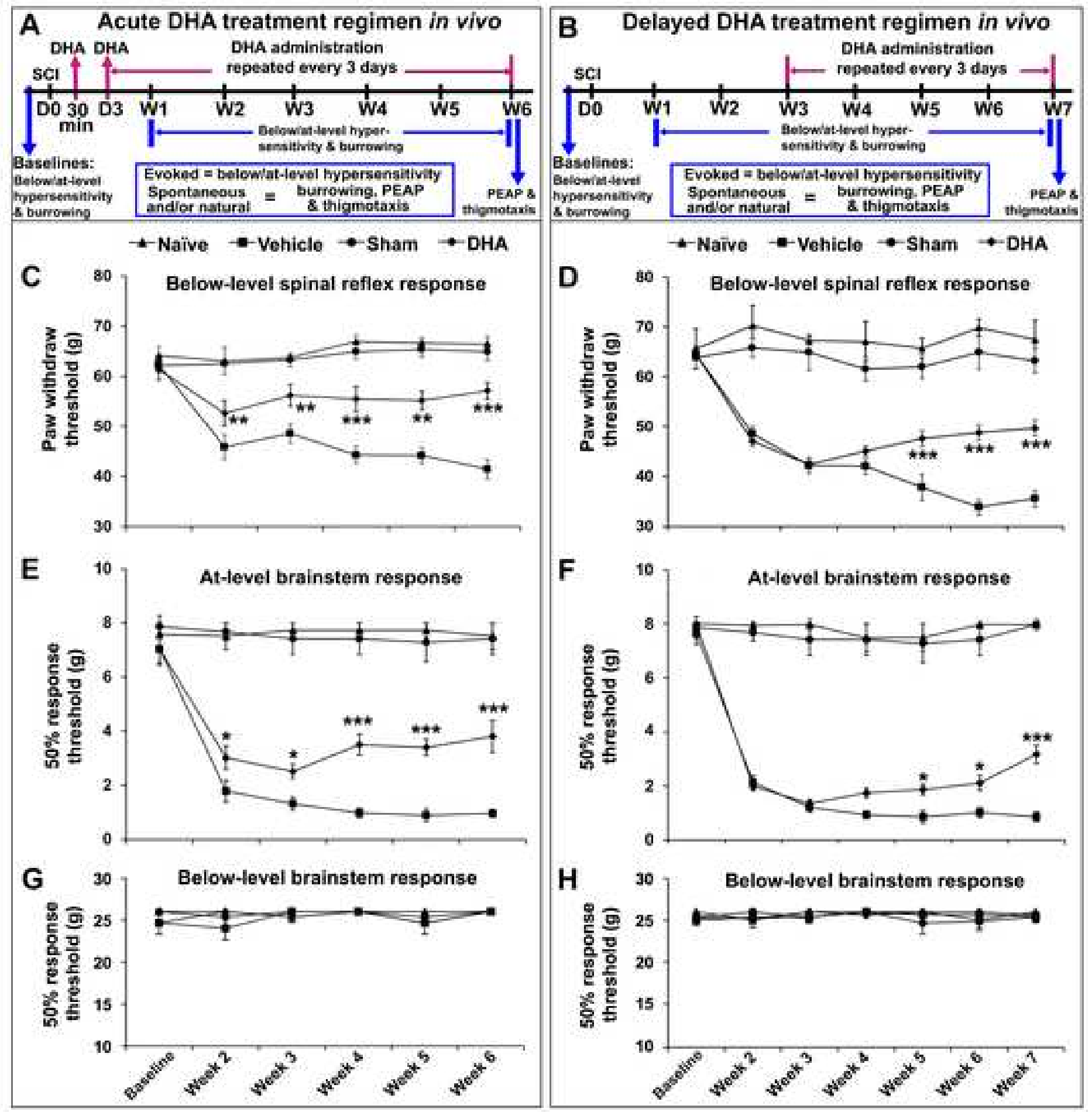

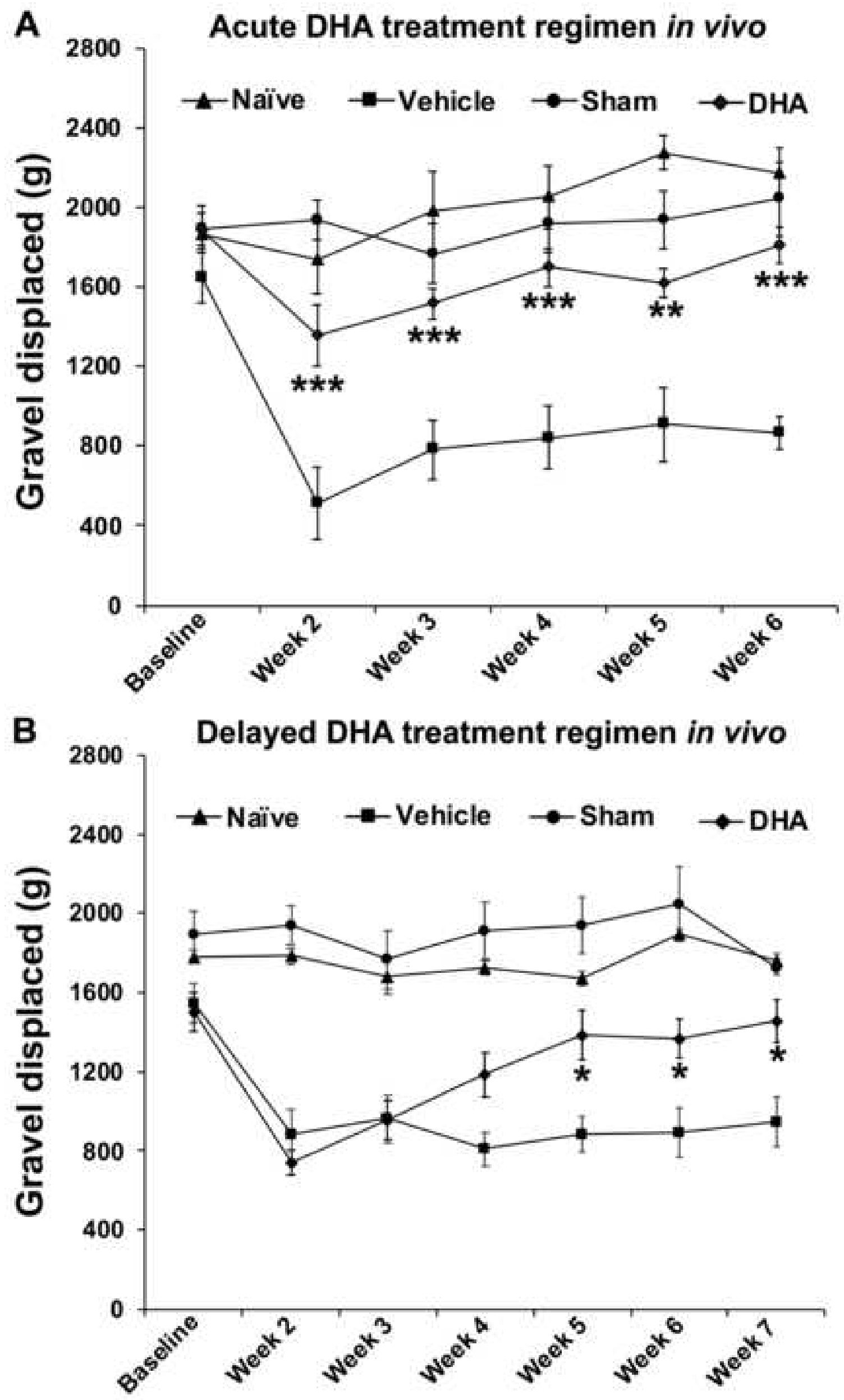


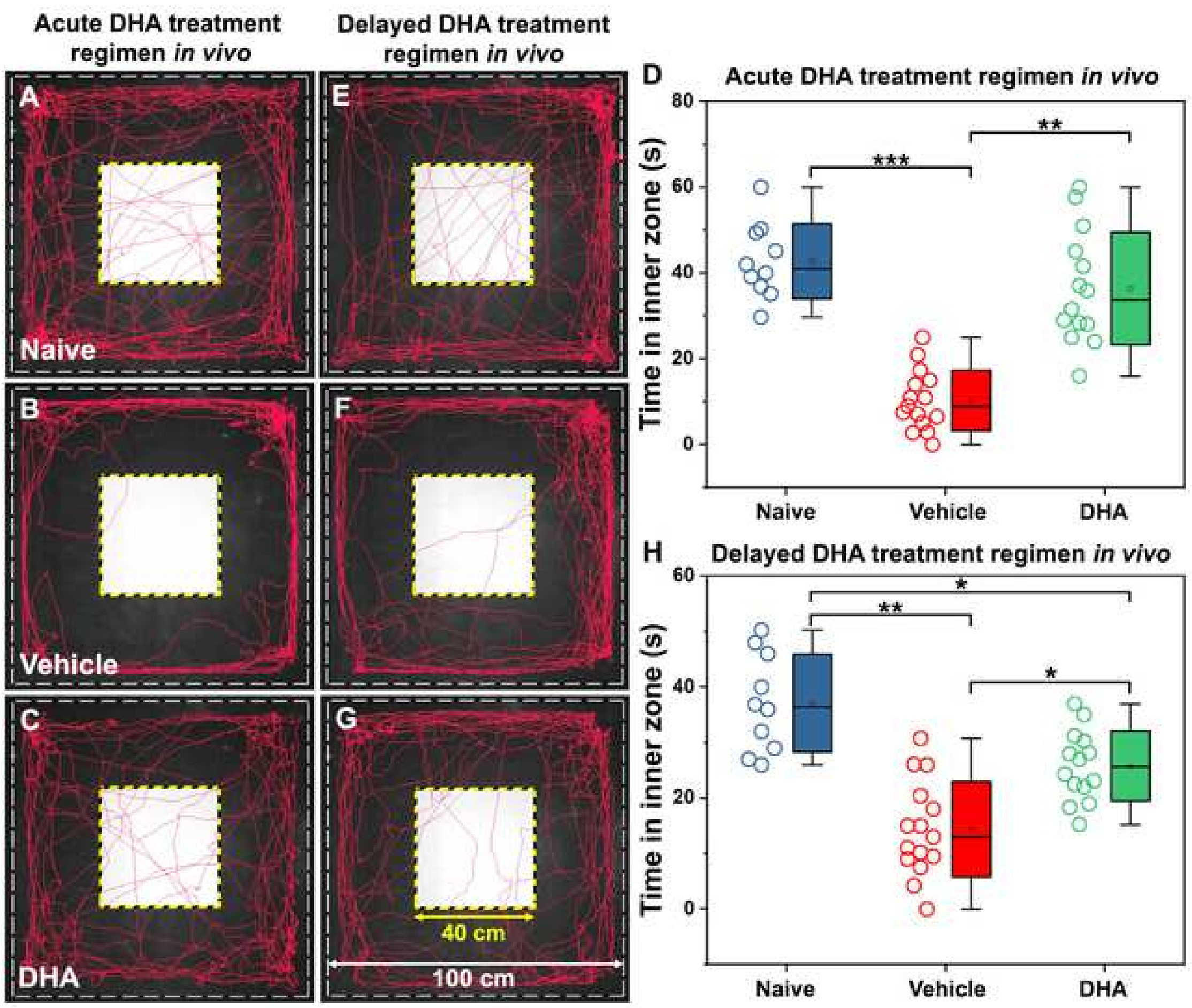




\section{Acute DHA treatment regimen in vivo}
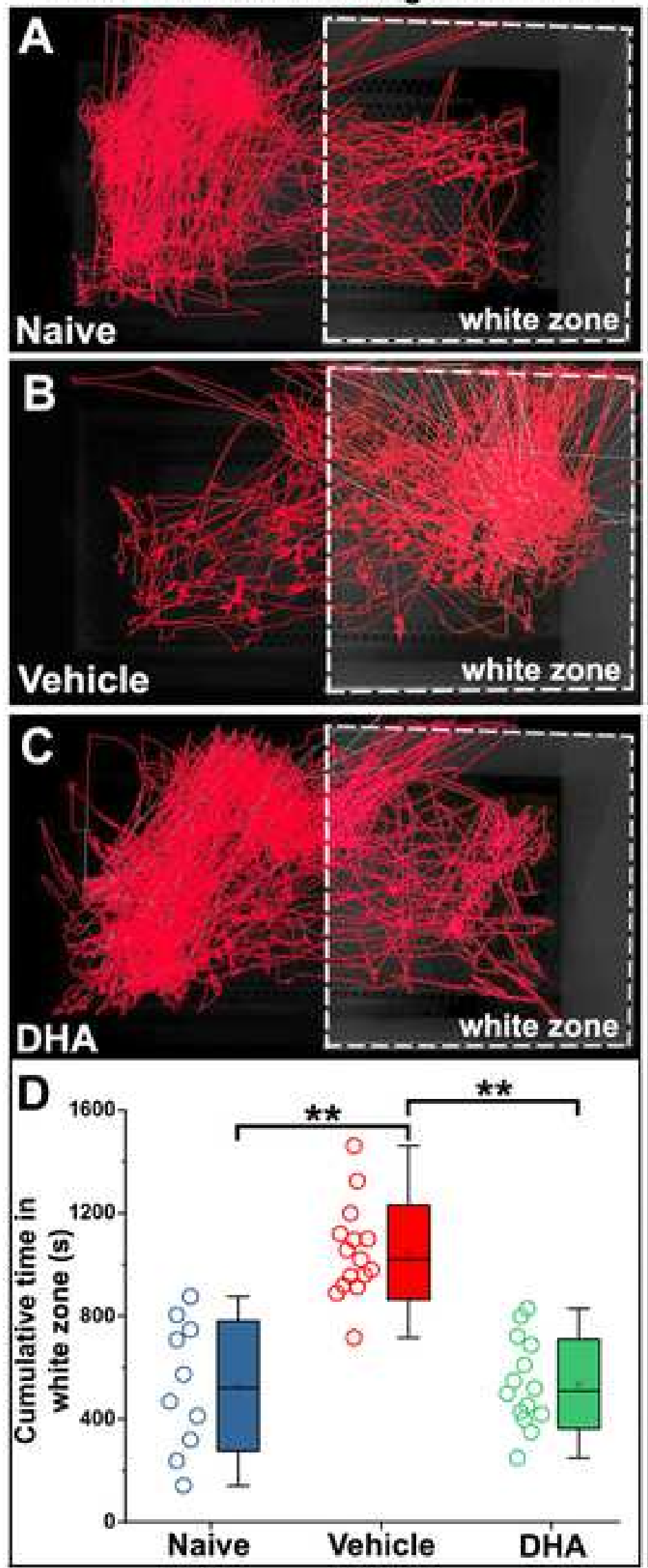

Delayed DHA treatment regimen in vivo
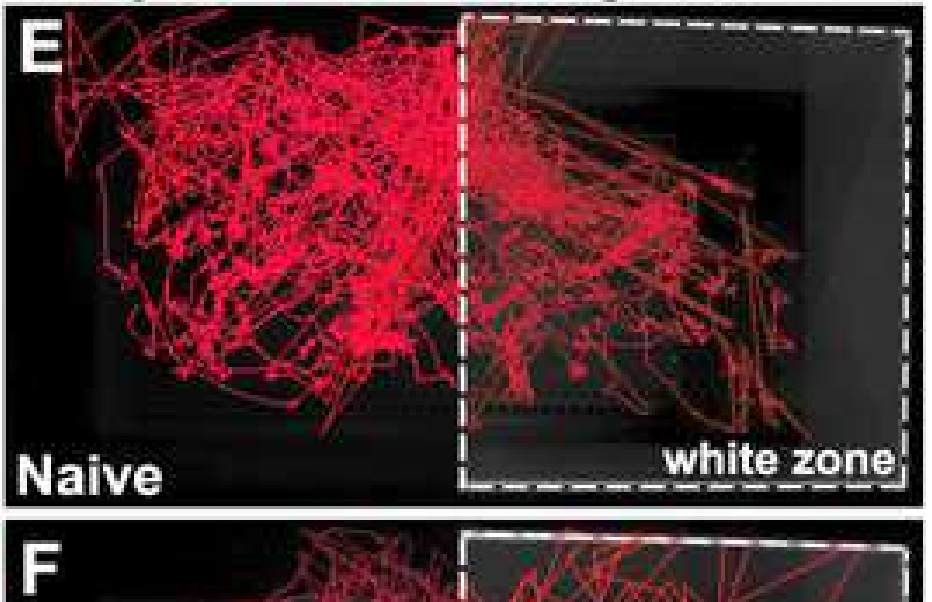

Vehicle

white zone

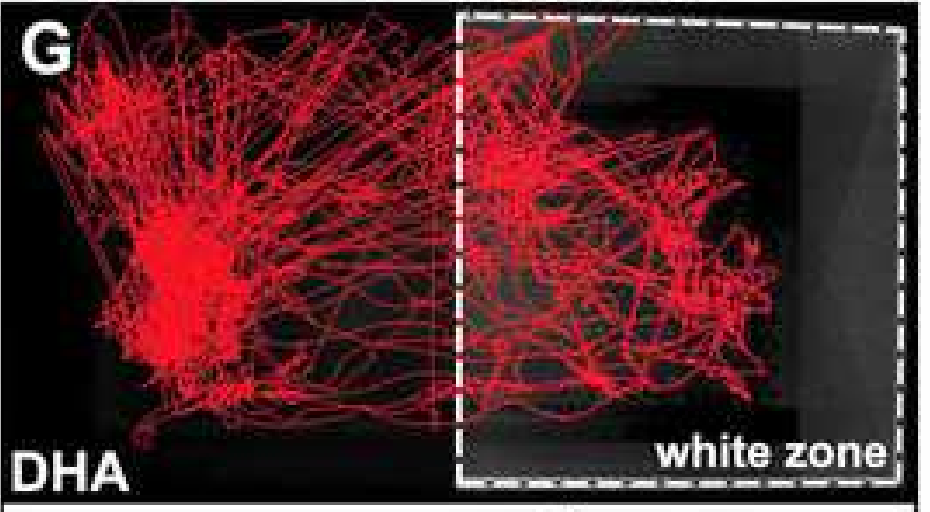

$\mathrm{H}$

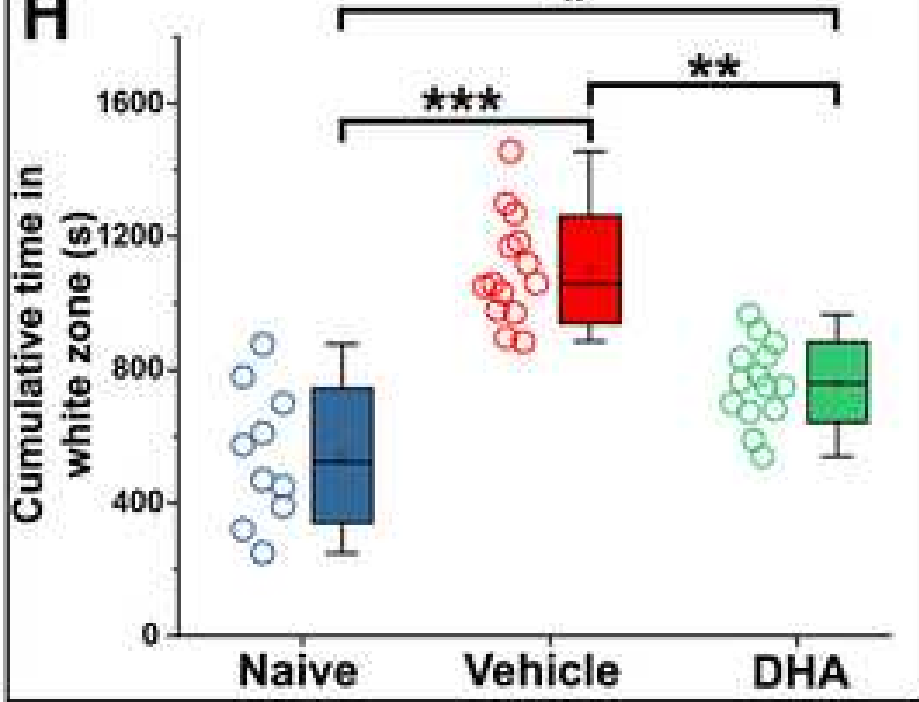




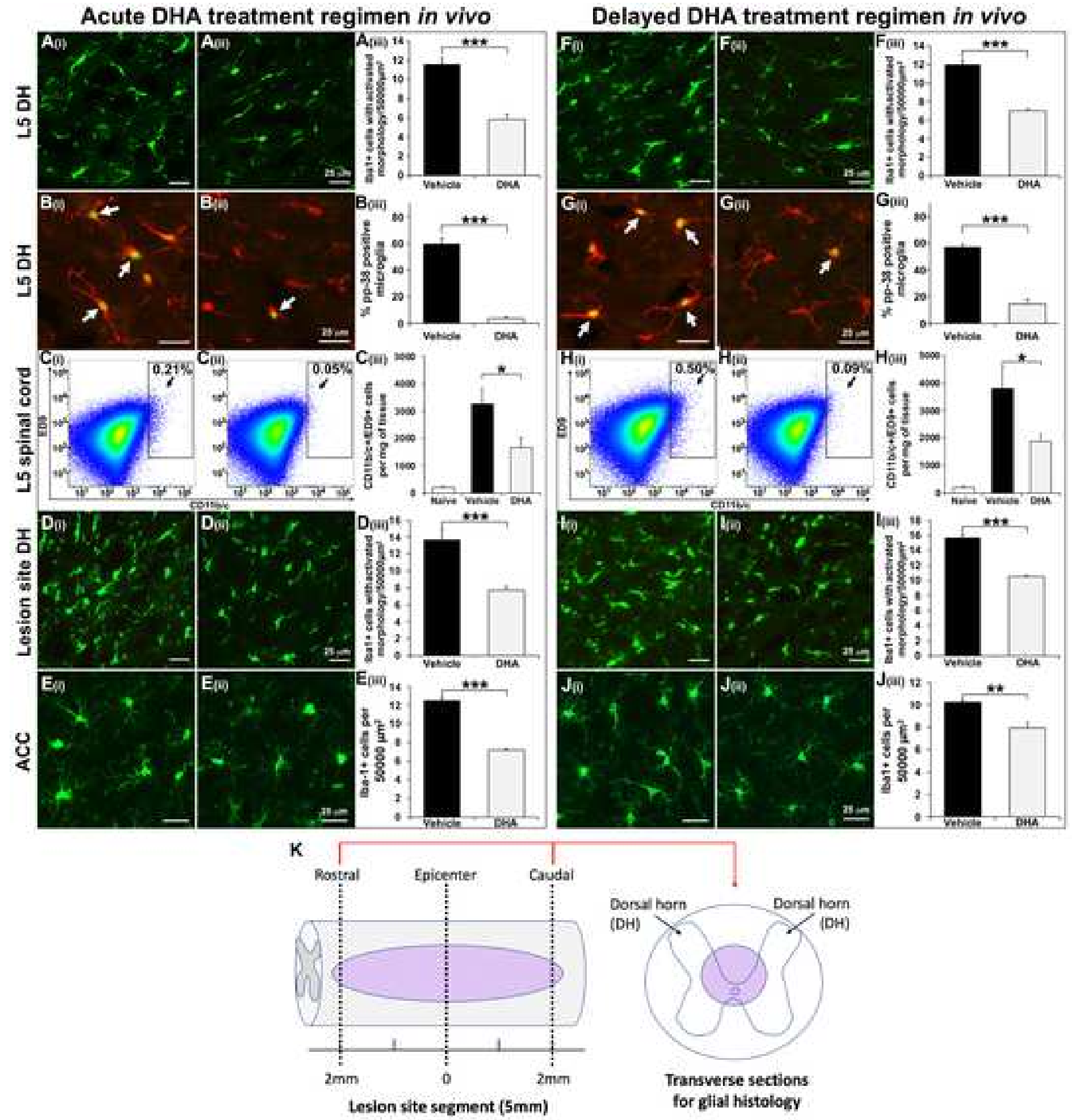




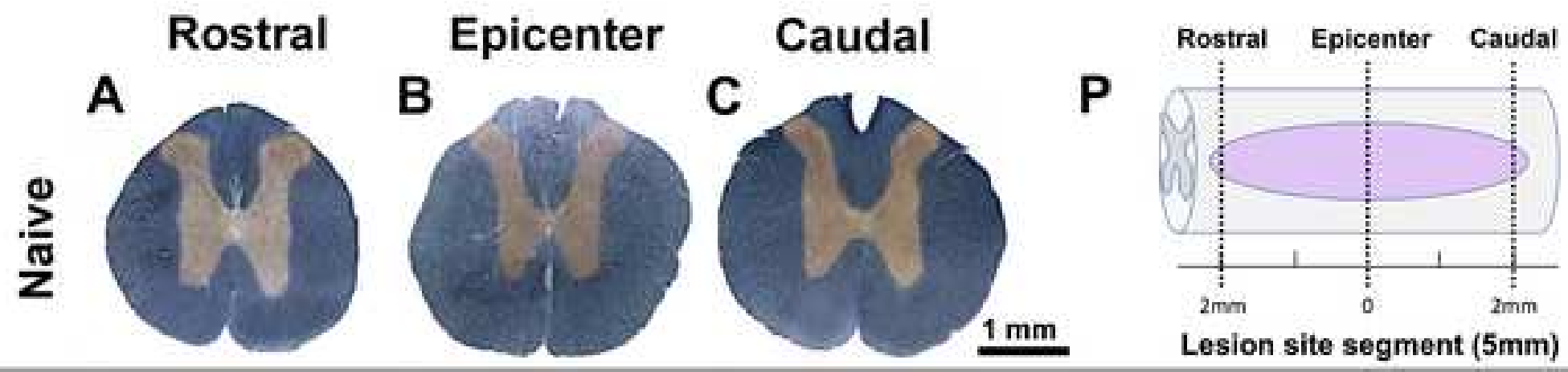

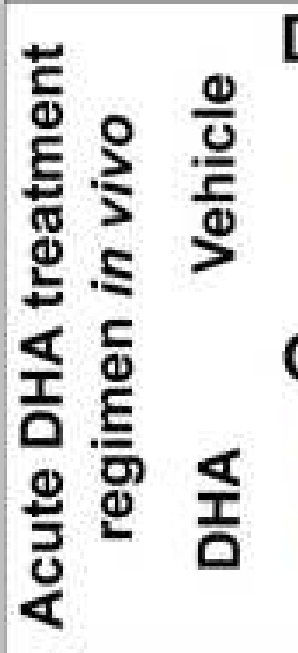

E

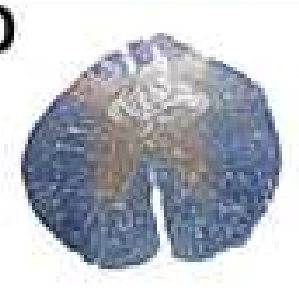

G

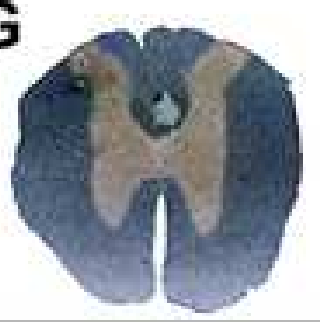

H I
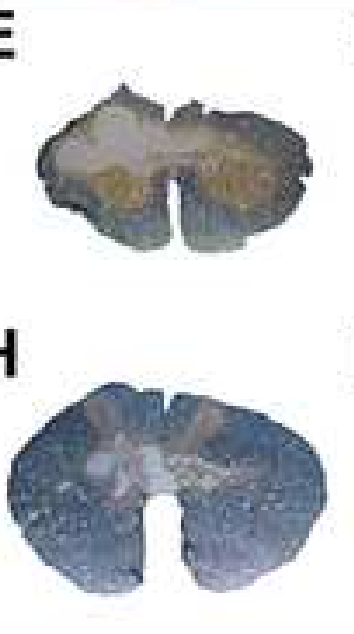

F

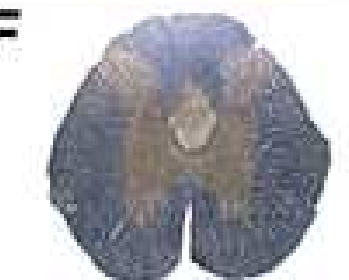

I
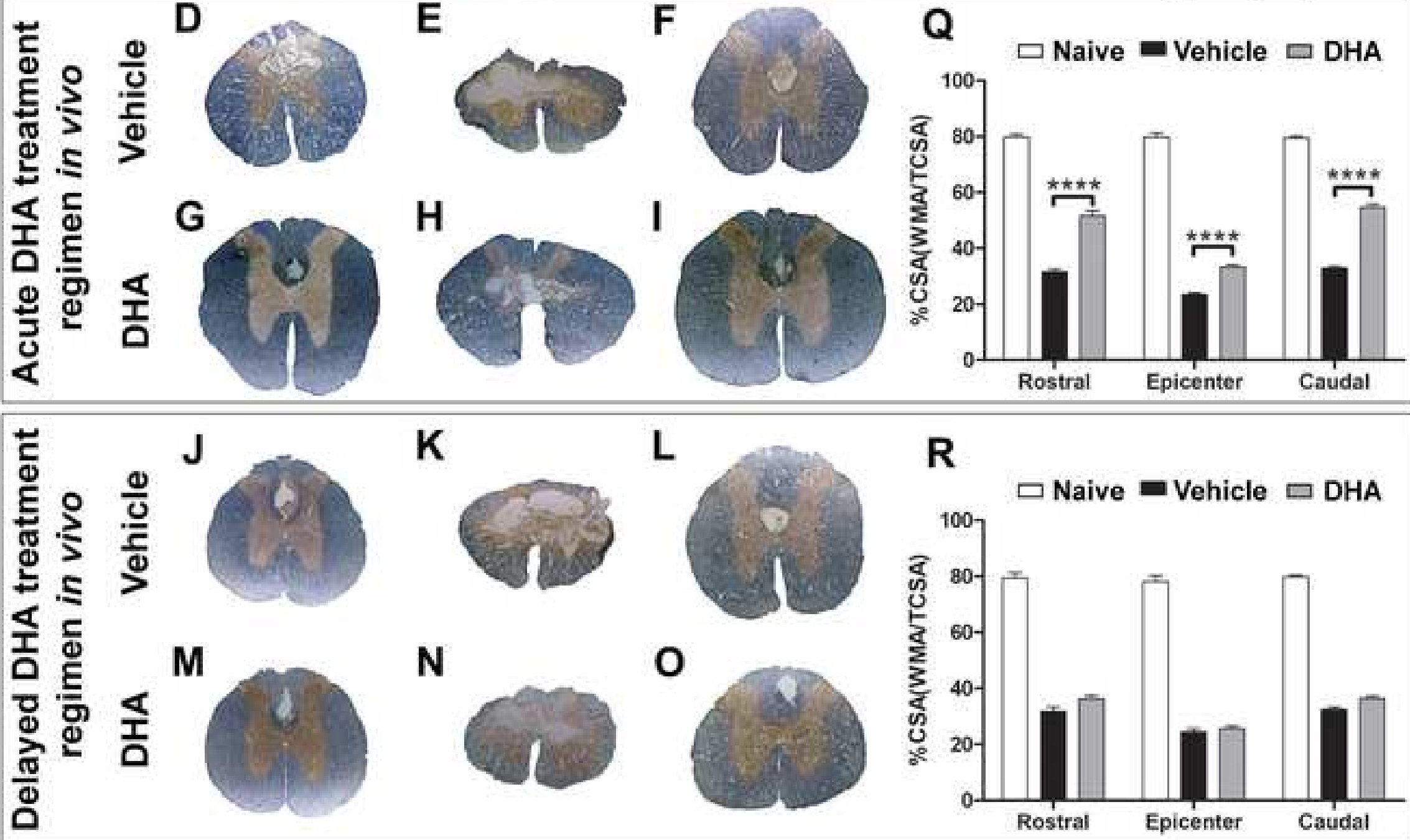

K

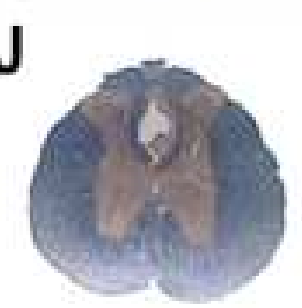

M

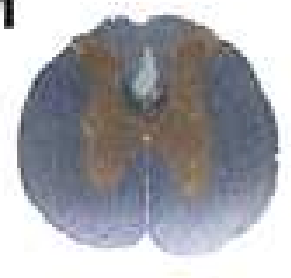

L

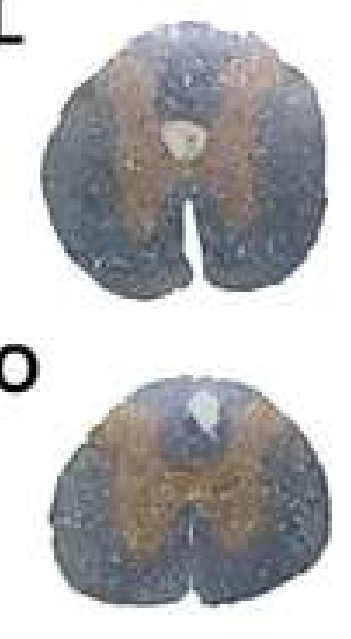

R

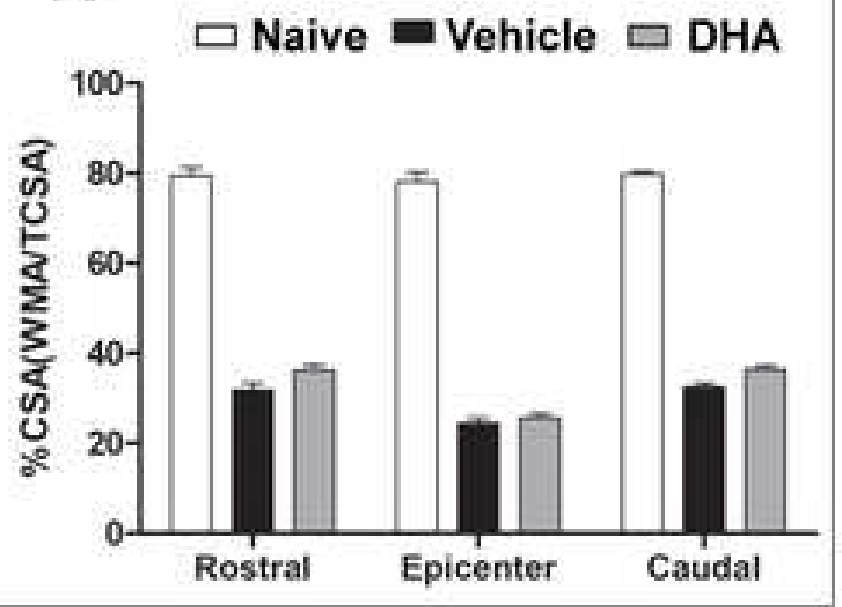


iNOS

TNF- $\alpha$

IL-1 $\beta$

IL-6
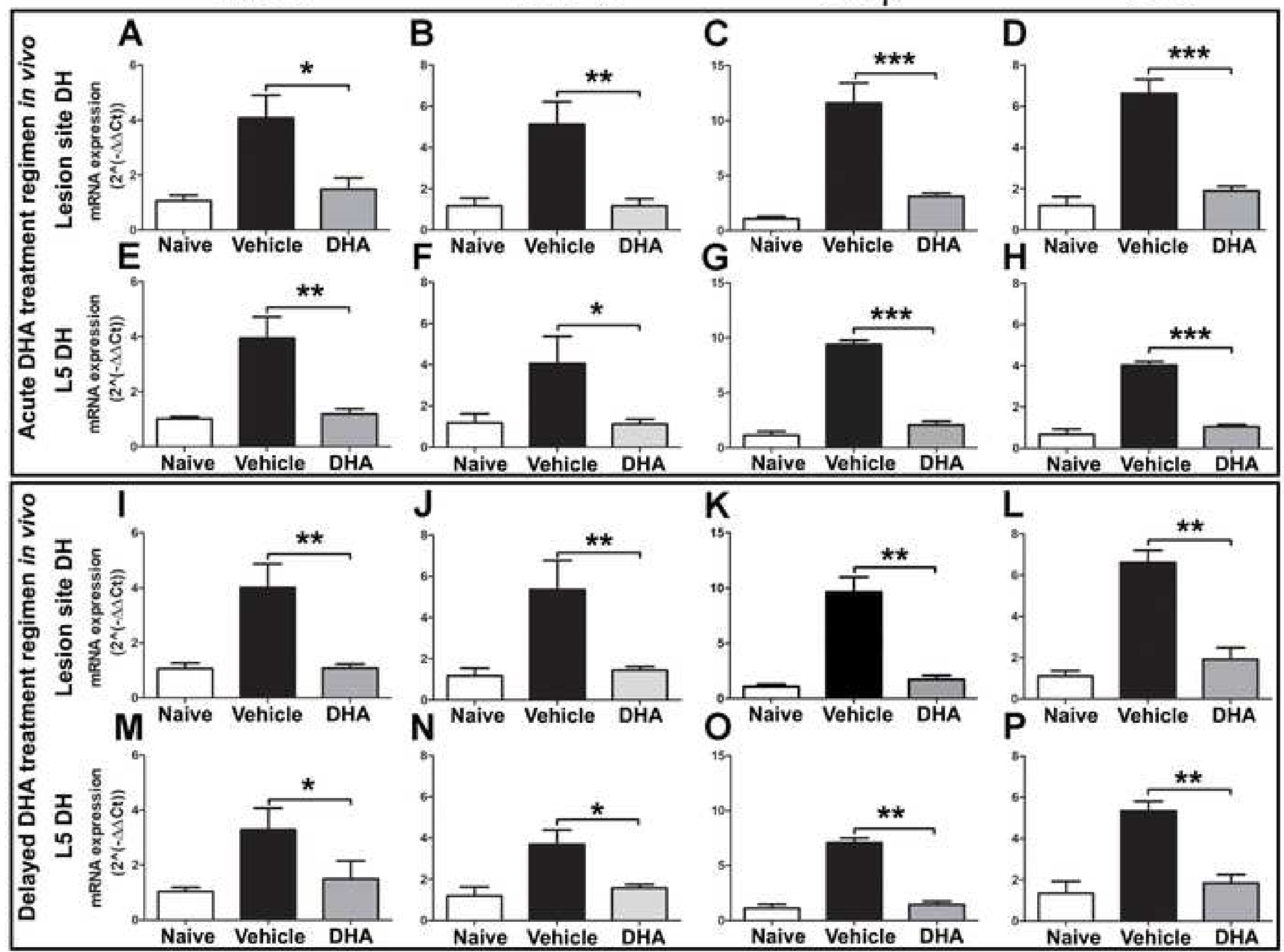


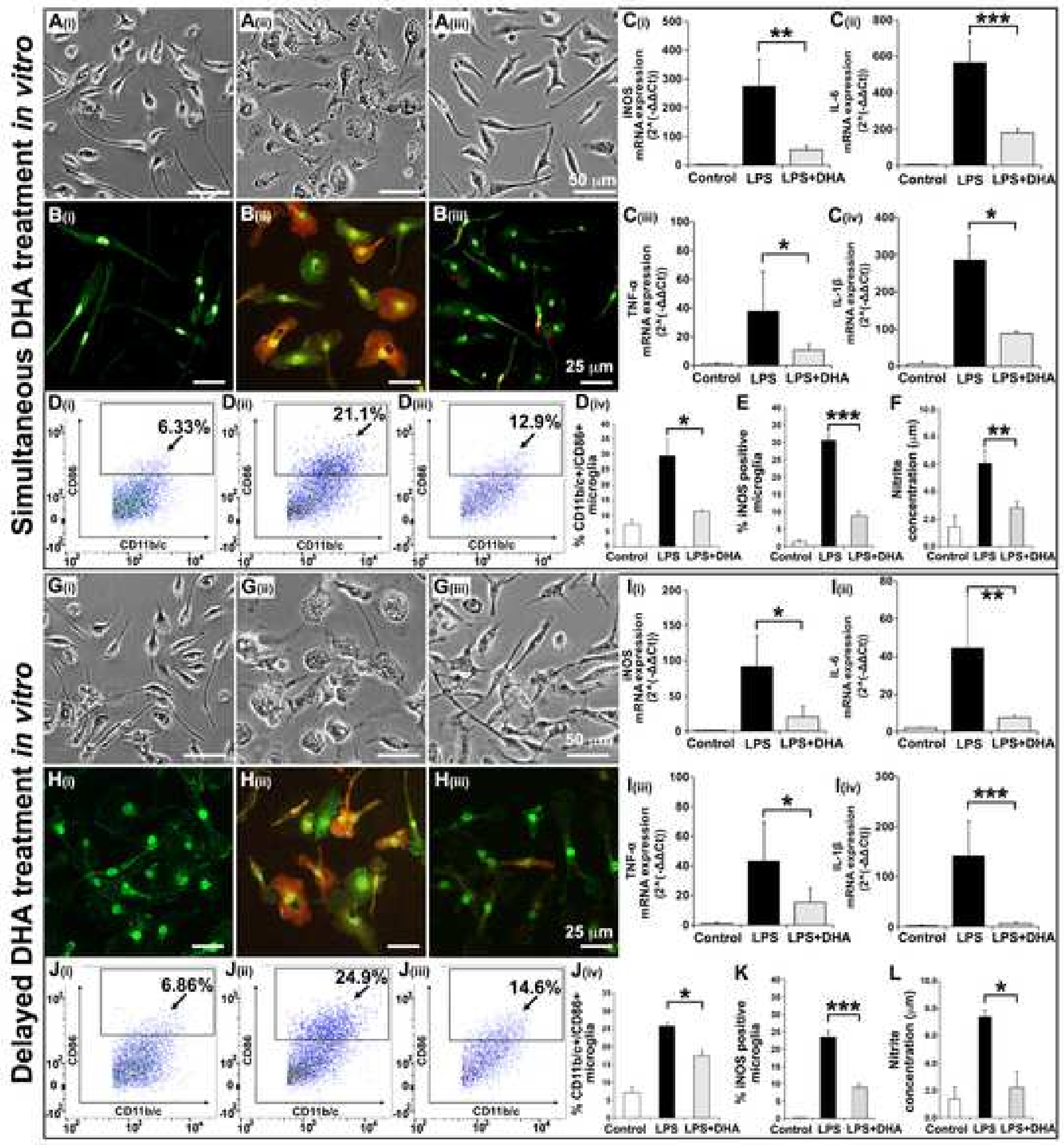



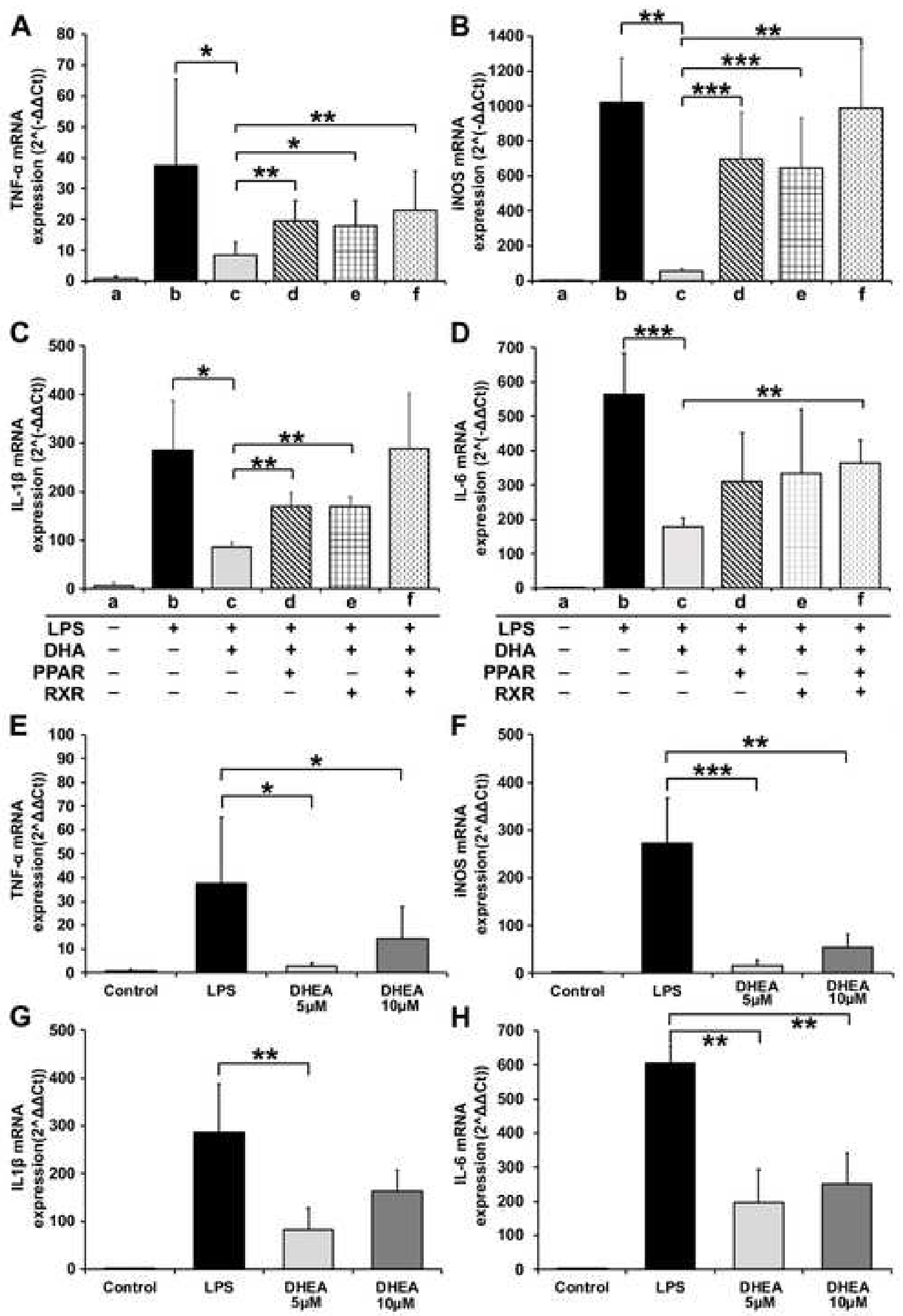
Table 1. Forward and reverse primers and their concentrations.

\begin{tabular}{|c|c|c|c|}
\hline Primer & Forward Primer 5' $\mathbf{- 3}$ & Reverse Primer 3' $\mathbf{- 5}$ & ' \\
& & & working \\
& & & $0.5 \mu \mathrm{M}$ \\
\hline TNF- $\boldsymbol{\alpha}$ & AGCCCACGTCGTAGCAAACCAC & AGGTACAACCCATCGGCTGGCA & $0.5 \mu \mathrm{M}$ \\
\hline iNOS & CACCTTGGAGTTCACCCAGT & ACCACTCGTATTGGGATGC & $0.4 \mu \mathrm{M}$ \\
\hline IL-6 & TCCTACCCCAACTTCCAATGCTC & TGGATGGTCTTGGTCCTTAGCC & $0.4 \mu \mathrm{M}$ \\
\hline IL-1 $\boldsymbol{\beta}$ & CACCTCTCAAGCAGAGCACAG & GGGTTCCATGGTGAAGTCAAC & $0.5 \mu \mathrm{M}$ \\
\hline YWHAZ & GCTACTTGGCTGAGGTTGCT & TGCTGTGACTGGTCCACAAT & 0.5 \\
\hline
\end{tabular}


Table 2. Behavioural outcome measures comparing Pregabalin-treated animals with other experimental groups.

Table 2 - Part A. At-level mechanical hypersensitivity, below-level mechanical hypersensitivity, and burrowing outcome measures.

\begin{tabular}{|c|c|c|c|c|c|c|c|c|c|}
\hline \multirow{3}{*}{$\begin{array}{c}\text { Behavioural outcome } \\
\text { measures }\end{array}$} & \multirow{3}{*}{$\begin{array}{c}\text { Treatment } \\
\text { Regimen }\end{array}$} & \multicolumn{8}{|c|}{ Behavioural assessment time points } \\
\hline & & \multicolumn{4}{|c|}{ Week 3 post-surgery (mean \pm SE) } & \multicolumn{4}{|c|}{ Week 6 post-surgery $($ mean \pm SE) } \\
\hline & & Naive & DHA & Vehicle & Pregabalin & Naive & DHA & Vehicle & Pregabalin \\
\hline \multirow{2}{*}{$\begin{array}{l}\text { At-level mechanical } \\
\text { hypersensitivity }\end{array}$} & Acute & $7.8 \pm 0.2^{\#}$ & $2.5 \pm 0.2$ & $1.3 \pm 0.1 *$ & $3.2 \pm 0.1$ & $7.5 \pm 0.5^{\#}$ & $3.9 \pm 0.6$ & $1.1 \pm 0.1^{*}$ & $5.1 \pm 0.5$ \\
\hline & Delayed & \multicolumn{4}{|c|}{$\mathrm{n} / \mathrm{a}$} & $7.9 \pm 0.1^{\#}$ & $2.2 \pm 0.3$ & $1.0 \pm 0.2 *$ & $4.2 \pm 0.4$ \\
\hline \multirow{2}{*}{$\begin{array}{c}\text { Below-level } \\
\text { mechanical } \\
\text { hypersensitivity }\end{array}$} & Acute & $63.5 \pm 1.3^{\#}$ & $55.5 \pm 2.1$ & $48.2 \pm 1.8^{*}$ & $58.0 \pm 2.1$ & $66.8 \pm 1.5^{\#}$ & $57.2 \pm 1.3$ & $41.1 \pm 1.6^{*}$ & $56.6 \pm 2.5$ \\
\hline & Delayed & \multicolumn{4}{|c|}{$\mathrm{n} / \mathrm{a}$} & $68.9 \pm 1.9^{\#}$ & $47.9 \pm 1.6$ & $33.4 \pm 1.4$ & $53.0 \pm 3.0$ \\
\hline \multirow{2}{*}{$\begin{array}{l}\text { Burrowing } \\
\text { behaviour }\end{array}$} & Acute & $\begin{array}{c}1980.7 \pm \\
199.5^{\dagger}\end{array}$ & $\begin{array}{c}1524.7 \pm \\
71.2\end{array}$ & $\begin{array}{l}797.9 \pm \\
135.4^{*}\end{array}$ & $\begin{array}{c}1379.5 \pm \\
138.8\end{array}$ & $\begin{array}{c}2173.0 \pm \\
128.2\end{array}$ & $\begin{array}{c}1816.8 \pm \\
85.5\end{array}$ & $\begin{array}{c}876.3 \pm \\
78.4^{*}\end{array}$ & $\begin{array}{c}1548.2 \pm \\
61.3\end{array}$ \\
\hline & Delayed & \multicolumn{4}{|c|}{$\mathrm{n} / \mathrm{a}$} & $\begin{array}{c}1888.2 \pm \\
43.1^{\ddagger}\end{array}$ & $\begin{array}{c}1371.3 \pm \\
93.3\end{array}$ & $\begin{array}{l}897.4 \pm \\
114.9^{*}\end{array}$ & $\begin{array}{c}1600.5 \pm \\
66.8\end{array}$ \\
\hline
\end{tabular}

Units are 50\% response threshold (g), paw withdrawal threshold (g) and gravel displaced (g) for at-level mechanical hypersensitivity, below-level mechanical hypersensitivity, and burrowing behaviour respectively. One-way ANOVA followed by Tukey-Kramer post-hoc multi-comparison adjustment was used for statistical analysis. ${ }^{*} \mathrm{p}<0.05$ vs naïve, DHA and Pregabalin. ${ }^{*} \mathrm{p}<0.05$ vs DHA, vehicle and Pregabalin. ${ }^{\dagger} \mathrm{p}<0.05 v s$ Pregabalin. ${ }^{\star} \mathrm{p}<0.05 v s$ DHA and Pregabalin. Sham data are not included, as they are similar to Naïve.

Table 2 - Part B. Thigmotaxis and PEAP outcome measures.

Behavioural outcome measures

Treatment
Regimen

\begin{tabular}{|c|} 
Behavioural assessment time points \\
\hline Week 6 (acute) or week 7 (delayed) post-surgery (mean $\pm \mathrm{SE})$ \\
\hline
\end{tabular}




\begin{tabular}{|c|c|c|c|c|c|c|}
\multicolumn{2}{c|}{} & & Naive & DHA & Vehicle & Pregabalin \\
\hline \multirow{3}{*}{ Thigmotaxis } & \multirow{2}{*}{ time in inner zone } & Acute & $42.8 \pm 2.8^{\dagger}$ & $36.4 \pm 3.5$ & $10.2 \pm 1.8^{*}$ & $22.8 \pm 3.8$ \\
\cline { 3 - 6 } & & Delayed & $38.3 \pm 2.8^{\dagger}$ & $26.6 \pm 1.7$ & $15.4 \pm 2.2^{*}$ & $26.0 \pm 2.5$ \\
\cline { 3 - 6 } & \multirow{2}{*}{ frequency into inner zone } & Acute & $19.7 \pm 1.1$ & $19.5 \pm 2.9$ & $7.6 \pm 2.1^{*}$ & $22.0 \pm 3.3$ \\
\cline { 3 - 6 } & & Delayed & $19.8 \pm 1.1$ & $19.7 \pm 2.1$ & $11.7 \pm 3.9^{*}$ & $20.8 \pm 2.7$ \\
\hline \multirow{3}{*}{ PEAP } & time in white zone & Acute & $529.6 \pm 79.9$ & $537.4 \pm 46.2$ & $1048.0 \pm 47.4^{*}$ & $608.0 \pm 59.9$ \\
\cline { 3 - 6 } & \multirow{2}{*}{ frequency into white zone } & Delayed & $542.0 \pm 64.2^{\#}$ & $762.6 \pm 31.2$ & $1102.3 \pm 41.6^{*}$ & $590.9 \pm 60.7$ \\
\cline { 3 - 6 } & & Acute & $87.9 \pm 12.0$ & $89.7 \pm 11.1$ & $138.7 \pm 20.3^{*}$ & $89.8 \pm 5.3$ \\
\cline { 3 - 6 } & & Delayed & $88.4 \pm 12.1^{\#}$ & $113.5 \pm 6.5$ & $146.9 \pm 12.0^{*}$ & $86.0 \pm 5.3$ \\
\hline
\end{tabular}

Units are seconds for Thigmotaxis-time in inner zone and PEAP-time in white zone., and entry numbers for Thigmotaxis-frequency into inner zone and PEAPfrequency into white zone. One-way ANOVA followed by Tukey-Kramer post-hoc multi-comparison adjustment was used for statistical analysis. * $<0.05 v s$ naïve, DHA and Pregabalin. ${ }^{\dagger} \mathrm{p}<0.05$ vs Pregabalin. ${ }^{\dagger} \mathrm{p}<0.05$ vs DHA and Pregabalin. ${ }^{\sharp} \mathrm{p}<0.05$ vs DHA. Sham data are not included, as they are similar to Naïve. 


\section{Supplementary Materials}

Fatty acid suppression of glial activation prevents central neuropathic pain after spinal cord injury

Marieta Georgieva ${ }^{1,3}$, Yuting $\mathrm{Wei}^{1}$, Maria Dumitrascuta ${ }^{1}$, Roger Pertwee $^{1}$, Nanna B. Finnerup ${ }^{2}$, Wenlong Huang ${ }^{1, *}$

${ }^{1}$ Institute of Medical Sciences, School of Medicine, Medical Sciences \& Nutrition, The University of Aberdeen, Foresterhill, Aberdeen, Scotland, AB25 2ZD, UK.

${ }^{2}$ Danish Pain Research Centre, Department of Clinical Medicine and Department of Neurology, Aarhus University Hospital, Aarhus, DK-8000, Denmark.

${ }^{3} 4 \mathrm{D}$ Pharma Research Ltd, Aberdeen, AB25 2ZS, UK

\section{*Corresponding author information:}

Dr Wenlong Huang, PhD, MBBS,

Institute of Medical Sciences

School of Medicine, Medical Sciences \& Nutrition

University of Aberdeen,

Foresterhill, Aberdeen, AB25 2ZD, UK

Telephone: +44 (0)1224 437290

Email:w.huang@abdn.ac.uk 
1. Materials and methods:

\subsection{In vivo study designs}

Supplementary Table 1: Details of groups, animal numbers in treatment groups and primary outcomes for behavioural experiments

\begin{tabular}{|c|c|c|c|c|c|c|}
\hline \multirow{2}{*}{$\begin{array}{l}\text { Experiment } \\
\text { Description }\end{array}$} & \multirow[b]{2}{*}{ Study type } & \multirow[b]{2}{*}{ Groups } & \multicolumn{2}{|c|}{ Group size } & \multirow{2}{*}{$\begin{array}{c}\text { Timeline of } \\
\text { behavioural } \\
\text { assessment }\end{array}$} & \multirow[b]{2}{*}{ Primary behavioural outcomes } \\
\hline & & & Initial & $\begin{array}{c}\text { After } \\
\text { exclusion }\end{array}$ & & \\
\hline \multirow{2}{*}{ BBB } & $\begin{array}{l}\text { Acute DHA } \\
\text { regimen }\end{array}$ & $\begin{array}{l}\text { Naïve/ Sham/ DHA/ Vehicle/ } \\
\text { Pregabalin }\end{array}$ & $10 / 10 / 15 / 15 / 12$ & $10 / 9 / 15 / 14 / 12$ & $\begin{array}{l}\text { D1, D3, D5, D7, W2, } \\
\text { W3, W4, W5, W6 }\end{array}$ & \multirow{2}{*}{ Locomotor functional recovery } \\
\hline & $\begin{array}{c}\text { Delayed DHA } \\
\text { regimen }\end{array}$ & $\begin{array}{l}\text { Naïve/ Sham/ DHA/ Vehicle/ } \\
\text { Pregabalin }\end{array}$ & $10 / 10 / 15 / 15 / 12$ & $10 / 10 / 14 / 14 / 12$ & $\begin{array}{l}\text { D1, D3, D5, D7, W2, } \\
\text { W3, W4, W5, W6, W7 }\end{array}$ & \\
\hline \multirow{2}{*}{$\begin{array}{l}\text { At-level mechanical } \\
\text { hypersensitivity }\end{array}$} & $\begin{array}{l}\text { Acute DHA } \\
\text { regimen }\end{array}$ & $\begin{array}{c}\text { Naïve/ Sham/ DHA/ Vehicle/ } \\
\text { Pregabalin } \\
\end{array}$ & $10 / 10 / 15 / 15 / 12$ & $10 / 9 / 15 / 14 / 12$ & W2, W3, W4, W5, W6 & \multirow{2}{*}{$\begin{array}{c}\text { Brainstem response (licking } \\
\text { guarding, biting, vocalizing, } \\
\text { jumping) in response to static } \\
\text { mechanical stimulus applied at the } \\
\text { level of the injury }\end{array}$} \\
\hline & $\begin{array}{l}\text { Delayed DHA } \\
\text { regimen }\end{array}$ & $\begin{array}{c}\text { Naïve/ Sham/ DHA/ Vehicle/ } \\
\text { Pregabalin }\end{array}$ & $10 / 10 / 15 / 15 / 12$ & $10 / 10 / 14 / 14 / 12$ & $\begin{array}{c}\text { W2, W3, W4, W5, } \\
\text { W6, W7 }\end{array}$ & \\
\hline \multirow{2}{*}{$\begin{array}{c}\text { Below-level } \\
\text { mechanical } \\
\text { hypersensitivity } \\
\text { (spinal reflex) } \\
\end{array}$} & $\begin{array}{l}\text { Acute DHA } \\
\text { regimen }\end{array}$ & $\begin{array}{l}\text { Naïve/ Sham/ DHA/ Vehicle/ } \\
\text { Pregabalin }\end{array}$ & $10 / 10 / 15 / 15 / 12$ & $10 / 9 / 15 / 14 / 12$ & W2, W3, W4, W5, W6 & \multirow{2}{*}{$\begin{array}{l}\text { Hind-paw withdrawal threshold in } \\
\text { response to static mechanical } \\
\text { stimulus applied on the later plantar } \\
\text { surface of the foot }\end{array}$} \\
\hline & $\begin{array}{l}\text { Delayed DHA } \\
\text { regimen }\end{array}$ & $\begin{array}{l}\text { Naïve/ Sham/ DHA/ Vehicle/ } \\
\text { Pregabalin }\end{array}$ & $10 / 10 / 15 / 15 / 12$ & $10 / 10 / 14 / 14 / 12$ & $\begin{array}{l}\text { W2, W3, W4, W5, } \\
\text { W6, W7 }\end{array}$ & \\
\hline \multirow{2}{*}{$\begin{array}{c}\text { Below-level } \\
\text { mechanical } \\
\text { hypersensitivity } \\
\text { (brainstem response) }\end{array}$} & $\begin{array}{l}\text { Acute DHA } \\
\text { regimen }\end{array}$ & $\begin{array}{c}\text { Naïve/ Sham/ DHA/ Vehicle/ } \\
\text { Pregabalin } \\
\end{array}$ & $10 / 10 / 15 / 15 / 12$ & $10 / 9 / 15 / 14 / 12$ & W2, W3, W4, W5, W6 & \multirow{2}{*}{$\begin{array}{c}\text { Brainstem response (licking } \\
\text { guarding, biting, vocalizing, } \\
\text { jumping) in response to static } \\
\text { mechanical stimulus applied at the } \\
\text { lateral plantar surface of the foot }\end{array}$} \\
\hline & $\begin{array}{l}\text { Delayed DHA } \\
\text { regimen }\end{array}$ & $\begin{array}{c}\text { Naïve/ Sham/ DHA/ Vehicle/ } \\
\text { Pregabalin }\end{array}$ & $10 / 10 / 15 / 15 / 12$ & $10 / 10 / 14 / 14 / 12$ & $\begin{array}{l}\text { W2, W3, W4, W5, } \\
\text { W6, W7 }\end{array}$ & \\
\hline \multirow{2}{*}{ Burrowing } & $\begin{array}{c}\text { Acute DHA } \\
\text { regimen }\end{array}$ & $\begin{array}{c}\text { Naïve/ Sham/ DHA/ Vehicle/ } \\
\text { Pregabalin }\end{array}$ & $10 / 10 / 15 / 15 / 12$ & $10 / 9 / 15 / 14 / 12$ & W2, W3, W4, W5, W6 & \multirow{2}{*}{$\begin{array}{c}\text { Grams of displaced gravel from the } \\
\text { burrows }\end{array}$} \\
\hline & $\begin{array}{c}\text { Delayed DHA } \\
\text { regimen }\end{array}$ & $\begin{array}{l}\text { Naïve/ Sham/ DHA/ Vehicle/ } \\
\text { Pregabalin }\end{array}$ & $10 / 10 / 15 / 15 / 12$ & $10 / 10 / 14 / 14 / 12$ & $\begin{array}{c}\text { W2, W3, W4, W5 } \\
\text { W6, W7 }\end{array}$ & \\
\hline \multirow{2}{*}{ PEAP } & $\begin{array}{l}\text { Acute DHA } \\
\text { regimen }\end{array}$ & $\begin{array}{l}\text { Naïve/ Sham/ DHA/ Vehicle/ } \\
\text { Pregabalin }\end{array}$ & $10 / 10 / 15 / 15 / 12$ & $10 / 9 / 15 / 14 / 12$ & W6 & \multirow{2}{*}{$\begin{array}{l}\text { Frequency of crossing to and time } \\
\text { spent in the white zone }\end{array}$} \\
\hline & $\begin{array}{l}\text { Acute DHA } \\
\text { regimen }\end{array}$ & $\begin{array}{l}\text { Naïve/ Sham/ DHA/ Vehicle/ } \\
\text { Pregabalin }\end{array}$ & $10 / 10 / 15 / 15 / 12$ & $10 / 10 / 14 / 14 / 12$ & W7 & \\
\hline
\end{tabular}




\begin{tabular}{|c|c|c|c|c|c|c|}
\hline \multirow{3}{*}{ Thigmotaxis } & $\begin{array}{c}\text { Delayed DHA } \\
\text { regimen }\end{array}$ & $\begin{array}{c}\text { Naïve/ Sham/ DHA/ Vehicle/ } \\
\text { Pregabalin }\end{array}$ & $10 / 10 / 15 / 15 / 12$ & $10 / 9 / 15 / 14 / 12$ & W6 & \begin{tabular}{c} 
Frequency of crossing and time \\
spent in the virtual inner zone \\
\cline { 2 - 6 }
\end{tabular} \\
$\begin{array}{c}\text { Delayed } \\
\text { regimen }\end{array}$ & $\begin{array}{c}\text { Naïve/ Sham/ DHA/ Vehicle/ } \\
\text { Pregabalin }\end{array}$ & $10 / 10 / 15 / 15 / 12$ & $10 / 10 / 14 / 14 / 12$ & W7 \\
\hline
\end{tabular}

D - Days post-injury / W - Weeks post-injury. 
Supplementary Table 2: Major domains of good laboratory practice to minimise the effects of experimental bias.

\begin{tabular}{|c|c|}
\hline & Description of procedures \\
\hline $\begin{array}{l}\text { Sample Size } \\
\text { Calculation }\end{array}$ & $\begin{array}{l}\text { - Group size was determined by sample size estimation for each experiment using } \\
\text { SigmaStat Version 3.5 (ANOVA sample size, desired power }=0.8 \text {, alpha }=0.05 \text { ). } \\
\text { - Effect sizes for estimation were derived from our previous studies (see below } \\
\text { Table S3). }\end{array}$ \\
\hline $\begin{array}{l}\text { Inclusion and } \\
\text { Exclusion Criteria }\end{array}$ & $\begin{array}{l}\text { - In experiments for at-level brainstem response, rats that developed a decrease in } \\
\text { the withdrawal threshold less than } 70 \% \text { from baselines were excluded. } \\
\text { - For burrowing, rats that burrowed less than } 500 \mathrm{~g} \text { during training and at baselines } \\
\text { were excluded. For PEAP, rats that spend less than } 20 \% \text { time in the white zone } \\
\text { were excluded. } \\
\text { For locomotor recovery, rats which scored } 2 \text { or more points on the BBB scale at } \\
\text { day } 0 \text { post-injury were excluded. Rats that did not reach a point of } 9 \text { on the BBB } \\
\text { scale by day } 14 \text { were also excluded. All rats excluded from BBB assessment were } \\
\text { also excluded for Horizontal ladder and Burrowing tests. }\end{array}$ \\
\hline Randomization & $\begin{array}{l}\text { - Animals were randomly assigned to sham, SCI vehicle-treatment, or SCI DHA- } \\
\text { treatment groups using random allocation software [10]. }\end{array}$ \\
\hline $\begin{array}{l}\text { Allocation } \\
\text { Concealment }\end{array}$ & $\begin{array}{l}\text { - The person performing SCI/sham surgeries and administering } \\
\text { DHA/Pregabalin/vehicle was unaware of the allocation to treatment group*. } \\
\text { - This was achieved by the blinding procedure described below. }\end{array}$ \\
\hline $\begin{array}{l}\text { Reporting of } \\
\text { Animals Excluded } \\
\text { from Analysis }\end{array}$ & $\begin{array}{l}\text { - Rats showing marked behavioural changes, exudates around wound, or sensitivity } \\
\text { to palpitation on handling attributable to surgery, drug, dosing procedure, } \\
\text { infection from surgery were excluded. } \\
\text { - Rats with significant surgical complications or whose general health deteriorates } \\
\text { were excluded. } \\
\text { - Any rat which showed a surgery-related weight of loss equal to more than } 25 \% \text { of } \\
\text { the body weight pre-injury was excluded. } \\
\text { The details of the number of excluded animals and the reason for exclusion are } \\
\text { stated in Supplementary Table } 1 \text {. }\end{array}$ \\
\hline Blinding procedures & $\begin{array}{l}\text { - Codes were assigned to different treatments by an independent person and kept in } \\
\text { a sealed envelope. The codes were not broken until the analysis had been } \\
\text { completed. } \\
\text { - The experimenter was 'blinded' to the treatments received and had no knowledge } \\
\text { of the experimental group to which an animal was randomized. }\end{array}$ \\
\hline
\end{tabular}

Animal exclusion: In the acute DHA regimen study, the following animals were excluded/culled: 1x sham-operated due to fluid build-up under the surgical incision and $1 \mathrm{x}$ vehicle-treated rat due to noninjury/surgically related fluid build-up in the lungs; In the delayed DHA regimen study the following animals were excluded/culled: 1x DHA-treated rat due to non-injury/surgically related foot wound and 
1x vehicle-treated rat due to non-injury/surgically related swollen and blocked gut. All conditions were reported to the animal facility vets and NACWO and registered with the Home Office.

Supplementary Table 3: Sample size estimation.

\begin{tabular}{|l|l|}
\hline Sample size & 15 \\
\hline Difference in means & 19 \\
\hline Standard deviation & 17.1 \\
\hline Number of groups & 5 \\
\hline Power & 0.80 \\
\hline Alpha & 0.05 \\
\hline
\end{tabular}

Based on Figure 7B (Baastrup et al., 2010, Pain 151:670-79)

\subsection{Locomotor functional recovery}

The recovery in the locomotor function of the rats following SCI was assessed by the commonly employed open field BBB scale [1]. Briefly, the BBB scoring scale used exclusively for the assessment of rat locomotor recovery, ranged between 0-21 points depending on the movements that the animal is able to perform, zero being complete paralysis and 21 for normal walking. A milestone in the rat's locomotor recovery is the score of 9 when it is able to support its body weight using its hind limbs, but still without having the ability to step. A score of 14 is another milestone in the functional recovery, when the rat is able to step in a coordinated manner.

Prior to surgeries the rats were acclimatized to a circular non-slip open field arena with $100 \mathrm{~cm}$ diameter and $60 \mathrm{~cm}$ height for three consecutive days and then baselines were taken. Each animal was allowed to 
walk freely in the arena for 4 minutes, while two observers assessed the rat's joint movements and walking abilities, and took notes of the performance in pre-designed BBB scoring template sheets. Following SCI surgeries hind limb paralysis was confirmed for each animal after its recovery from anesthesia. BBB assessment was performed at days 1, 3, 5, 7 after injury and weekly thereafter until the end of the studies. Analysis of the recorded scores were performed blindly by another member of the laboratory group in reference to the BBB scale descriptions [1].

\subsection{Greiss assay}

In order to measure the release of nitrite in the medium of microglia cells activated with LPS or receiving LPS plus simultaneous or delayed DHA treatment, we performed a Greiss assay, as described previously [11]. Briefly, following treatments the medium in which the cells were incubated for the particular duration of time was collected in sterile Eppendorf tubes and stored at $-80^{\circ} \mathrm{C}$. Greiss assay reagents were prepared as follows: Greiss reagent A (1\% sulphanilamide (1mg; Sigma, UK) in 5\% orthophosphoric acid (100 ml; Sigma, UK); Greiss reagent B (0.1\% naphthylethylenediamine (100 mg; Sigma, UK) and $5 \%$ orthophosphoric acid $\left(100 \mathrm{ml}\right.$; Sigma, UK). Stock solution of $1 \mathrm{mM}$ sodium nitrite $\left(\mathrm{Na}-\mathrm{NO}_{2}, 69.00\right.$ $\mathrm{g} / \mathrm{mol}$ ) was prepared by dissolving $0.345 \mathrm{mg}$ sodium nitrate (Sigma, UK) in $5 \mathrm{ml}$ serum free DMEM media. Standard curve of sodium nitrite from 0-100 $\mu \mathrm{M}$ was prepared in base media. The media samples were defrosted. Standard curve and test sample were aliquoted in $100 \mu 1$ triplicates into a 96-well plate. The Greiss reagent A and B were mixed in a 1:1 ration and $100 \mu 1$ were added to each well. The samples were immediately quantified for change in absorbance at $544 \mathrm{~nm}$ using the Galaxy Fluorstar spectrophotometer (BMG Labtech, UK).

\section{Supplementary results:}

\subsection{DHA treatment regimens result in improved locomotor functional recovery}


The neuroprotective effects of DHA and its potential to improve locomotor functional recovery in different animal models of SCI, such as the static compression model, have been previously reported [4; 5; 8]. Thus, we used the BBB locomotor scale, as described in the literature [5; 7] to follow up the locomotor functional recovery of the rats with SCI. During the baseline measures, rats from different experimental groups obtained a score of 21 in accordance to the BBB scale (Supplementary Fig. 1a-b), reflecting on their unimpaired locomotor abilities. Following surgeries, rats with SCI developed significant locomotor disability in comparison to the naïve and sham operated animals, which was evident by marked reductions in the BBB scores in a range between 0 and 2 points in the first 3 days (Supplementary Fig. 1a-b). The locomotor deficits of SCI rats receiving vehicle treatment were continuous through the duration of both studies. Our analysis showed that there was a significant main effect of treatment on the BBB scores by the acute DHA treatment regimen $\left(\mathrm{F}_{(1,27)}=40.52, \mathrm{p}=0.000012\right.$, two-way repeated-measures ANOVA), in which animals receiving DHA treatment significantly improved their BBB scores (i.e. more than 2 scores) when compared to those of SCI rats treated with vehicle, from week 2 post-surgery onwards (Supplementary Fig. 1a). We also found a significant main effect of time on the BBB scores $\left(\mathrm{F}_{(8,216)}=1196.44, \mathrm{p}=0.0000001\right)$ and a significant treatment with time interaction $\left(\mathrm{F}_{(8,216)}=9.52, \mathrm{p}=0.0011\right)$. In order to provide evidence that the improved locomotor recovery following SCI of the rats treated with DHA is a result of the neuroprotective properties of the compound, we collected spinal cord longitudinal sections from naïve, SCI vehicle-treated, and SCI DHA-treated rats and performed an immunostaining against GFAP and Laminin (an extracellular matrix marker), in order to assess the lesion cavity appearance. We demonstrated that the lesion cavities of rats that were given the acute DHA regimen appeared to be smaller than those of SCI rats treated with vehicle and the accumulation of laminin around the injury site also appeared to be reduced (Supplementary Fig. 7b, c).

We also assessed whether the delayed DHA treatment regimen would have an effect on the locomotor recovery of rats with SCI. Two-way repeated-measures ANOVA showed significant main effects of 
treatment $\left(\mathrm{F}_{(1,26)}=13.75, \mathrm{p}=0.001\right)$ and time $\left(\mathrm{F}_{(3,78)}=60.89, \mathrm{p}=0.000057\right)$ on the BBB scores by the delayed DHA treatment regimen. Moreover, the analysis showed a significant treatment with time interaction $\left(F_{(3,78)}=10.99, p=0.0017\right)$. We found that delayed DHA treatment led to significant improvement of locomotor functional recovery of SCI rats in weeks 6 and 7 post-surgery, when compared to that of vehicle-treated SCI rats (Supplementary Fig. 1b; p=0.0001, Tukey-Kramer post hoc). Our immunohistochemistry of GFAP and laminin staining did not show that there appeared to be any differences in the lesion cavity size of rats receiving delayed DHA treatment and rats receiving vehicle treatment following SCI surgeries (Supplementary Fig. 7d, e).

\subsection{Effects of locomotor functions on complex pain-related outcome measures}

We used BBB locomotor scale to monitor the hindlimb locomotor function on a weekly basis from week 2 post-surgery throughout the two DHA treatment regimen studies. We found that the locomotor functional recovery is in line with the ability of animals to enter the burrows. We observed that at week 2 post-surgery in the acute regimen study, vehicle-treated SCI rats already achieved occasional weightsupported plantar stepping, and continued to improve with frequent to consistent weight-supported plantar stepping from week 3 onwards; whilst DHA-treated SCI rats already achieved frequent to consistent weight-supported plantar stepping at week 2 (Supplementary Fig. 1). In the delayed regimen study, all SCI rats reached occasional weight-supported plantar stepping at week 2 and frequent to consistent weight-supported plantar stepping from week 3 onwards (Supplementary Fig. 1). Therefore, the locomotor recoveries from both regimen studies allowed all SCI rats from week 2 onwards to enter the burrows without difficulty and we found that SCI rats used both forepaws and hindpaws to displace gravels. For thigmotaxis and PEAP, all SCI rats achieved consistent weight-supported stepping at week 6 or week 7, therefore they can readily explore the open field arena and white/black zones. 


\subsection{DHA significantly decreases the activation of astrocytes in vitro and in vivo}

We also investigated the effects of DHA on astrocyte activation. DHA $(0.8 \mu \mathrm{M})$ was found as the most efficacious in reducing GFAP-expression $\left(\mathrm{F}_{(5,68)}=44.25, \mathrm{p}=0.00045\right.$, one-way ANOVA; Supplementary Fig. 4b) in cultured astrocytes co-treated with LPS and DHA for 4 hours; DHA also preserved nonreactive morphology (Supplementary Fig. 5). With qPCR, we showed that mRNA expression of iNOS, TNF- $\alpha$, IL-1 $\beta$, and IL-6 in cultured astrocytes was significantly reduced by DHA when compared to control (co-treatment with LPS/DHA: $\mathrm{p}=0.0009-0.0021$; pre-activation with LPS followed by DHA treatment: $\mathrm{p}=0.0010-0.0054$; t-tests in R; Supplementary Fig. 6).

With immunohistochemistry, we then elucidated that GFAP immunoreactivity in the dorsal horns and ACC was significantly reduced by systemic DHA treatments compared to vehicle-control [acute regimen: L5 ( $\mathrm{p}=0.00017$, Supplementary Fig. 7Ai-iii), lesion site ( $\mathrm{p}=0.00036$, Supplementary Fig. 7Biiii), ACC ( $p=0.00091$, Supplementary Fig. 7Ci-iii); delayed regimen: L5 ( $p=0.0035$, Fig. Supplementary Fig. 7Di-iii), lesion site ( $\mathrm{p}=0.00019$, Supplementary Fig. 7Ei-iii), ACC ( $\mathrm{p}=0.0038$, Supplementary Fig. 7Fi-iii); Mann-Whitney test]. 


\section{Supplementary Figures:}
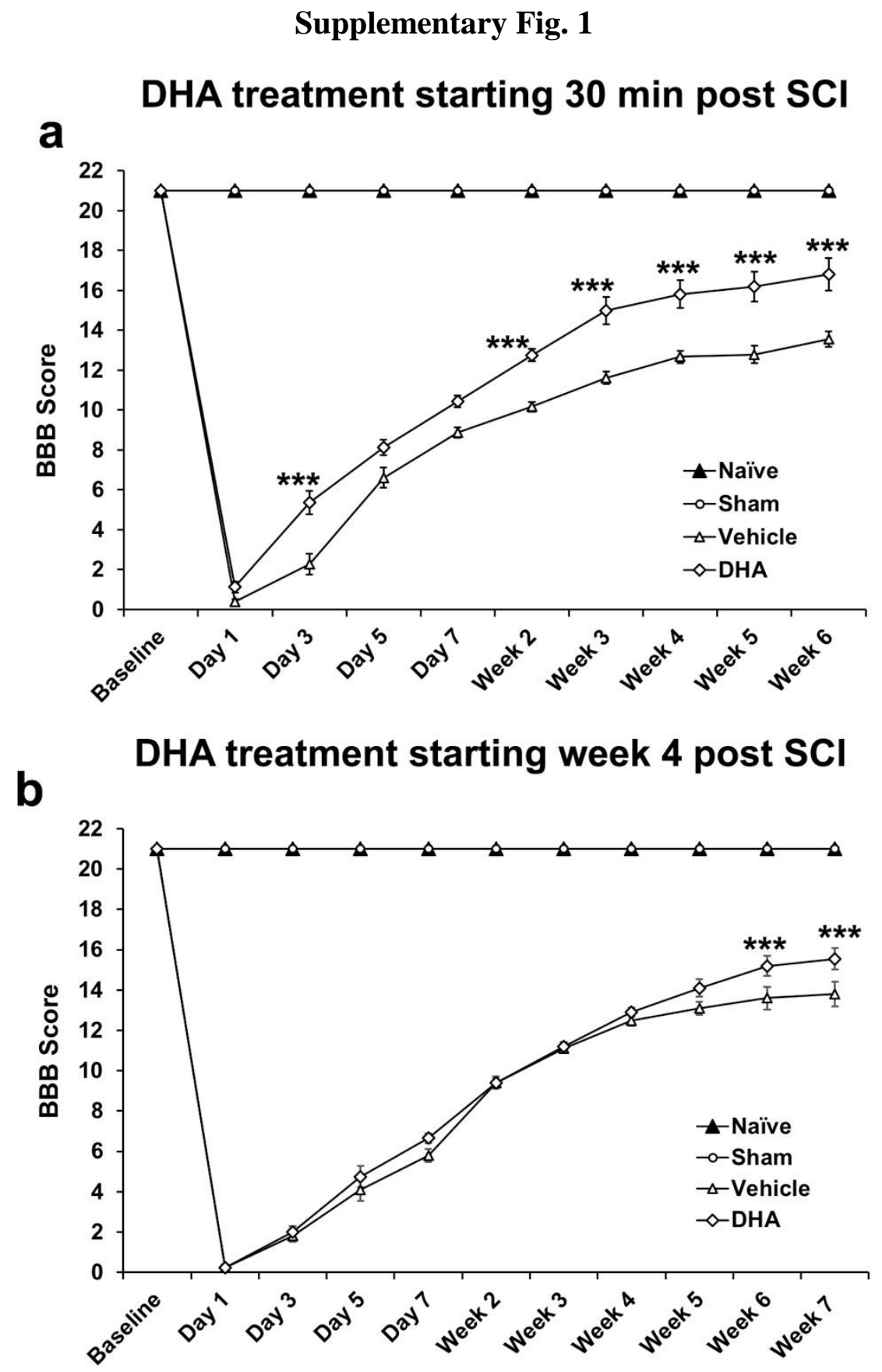

Supplementary Fig. 1. Effects of systemic DHA treatment on locomotor function recoveries. BBB locomotor assessment revealed that: (a) DHA treatment administered systemically every 3 days starting 30 minutes post-surgery (acute administration) significantly improved the locomotor functional recoveries of SCI rats between week 2 and week 6 , when compared to those of the vehicle-treated group. 
(b) DHA treatment administered systemically every 3 days starting 4 weeks post-surgery (chronic administration) improved the functional recoveries of SCI rats in week 6 and week 7, when compared to those of the vehicle-treated group. Each value is the mean \pm SEM. N=10-15 per treatment group. Twoway repeated-measures ANOVA was used to determine the main effects of treatment (DHA vs vehicle) and time (weekly). Statistical significance of the differences between the groups was determined by oneway ANOVA followed by Tukey-Kramer post hoc test at each week point; ***p<0.001 vs vehicle. 
Supplementary Fig. 2
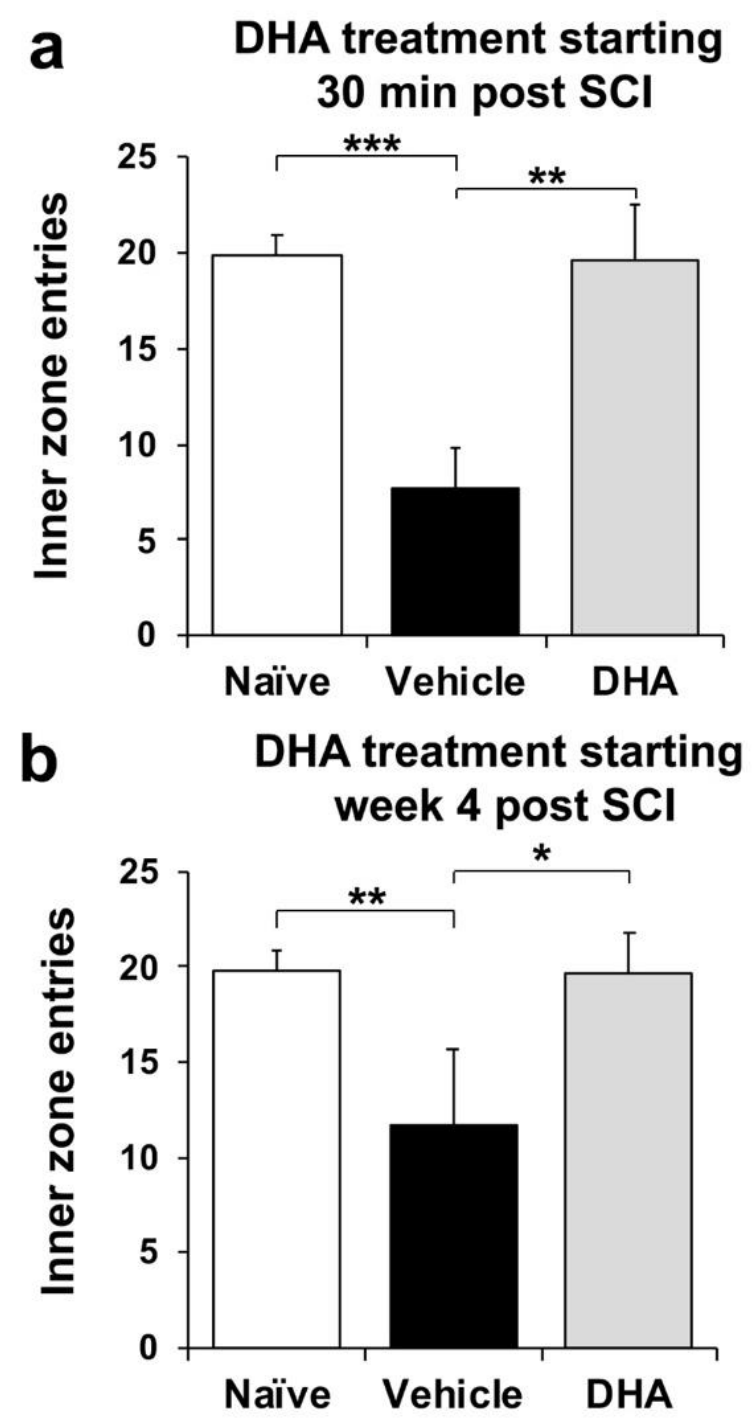

Supplementary Fig. 2. The effect of systemic DHA treatment on the frequency of entry in the virtual inner zone during the assessment of thigmotactic behaviour at week 6 and week 7. (a) The number of entries to the virtual inner zone of SCI rats at week 6 post-surgery was markedly decreased, when compared to that of the naïve group. DHA treatment in SCI rats commencing 30 minutes postsurgery and continued every 3 days thereafter for 6 weeks, significantly increased the frequency of crossing into the inner zone, when compared to that of vehicle-treated SCI rats. (b) SCI rats at week 7 post-injury crossed the virtual inner zone significantly fewer times than naïve rats. Delayed DHA 
treatment in SCI rats administered 4 weeks post-surgery and its continued administration every 3 days thereafter for 4 weeks, significantly increased the frequency of entries into the inner zone, when compared to that of vehicle-treated SCI rats. Data are presented as the mean \pm SEM. N=10-15 per treatment group. One-way ANOVA and Tukey-Kramer post hoc test were used for statistical analysis; ${ }^{*} \mathrm{p}<0.05, * * \mathrm{p}<0.01$, or $* * * \mathrm{p}<0.001$ vs vehicle. 
Supplementary Fig. 3

a DHA treatment starting $30 \mathrm{~min}$ post $\mathrm{SCl}$

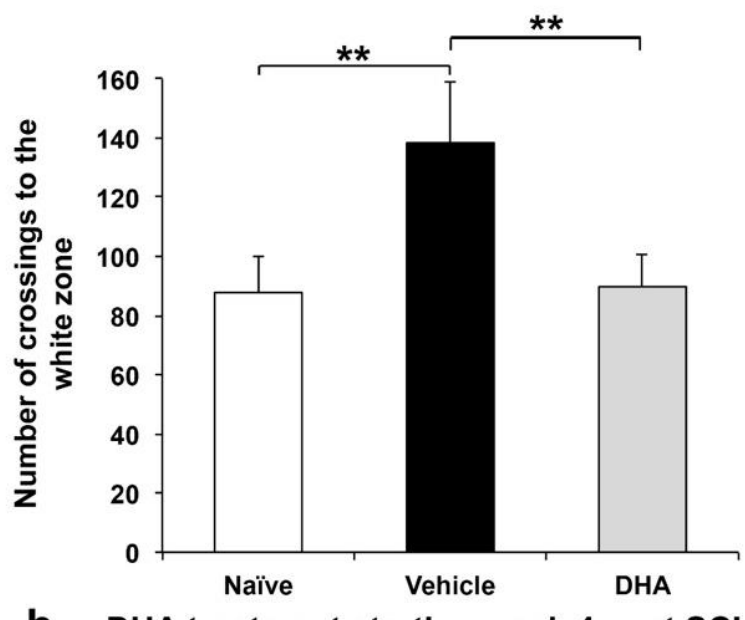

b DHA treatment starting week 4 post SCI

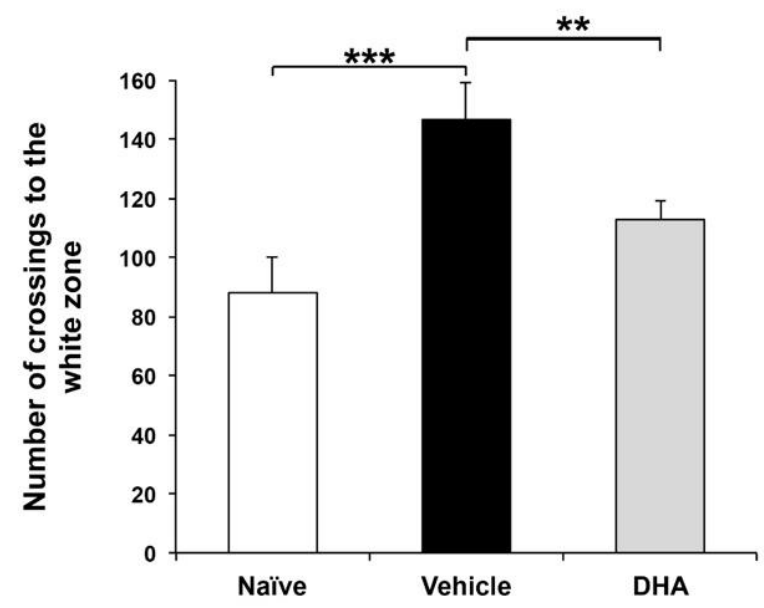

Supplementary Fig. 3. The effect of systemic DHA treatment on the number of crossing to the white zone of the PEAP box at week 6 and week 7. (a) The number of crossings to the white zone of the PEAP box of SCI rats at week 6 post-surgery was significantly increased, when compared to that of the naïve group. DHA treatment administered acutely post-surgery, significantly decreased the frequency of crossing into the white zone of the arena, when compared to that of vehicle-treated SCI rats. (b) The frequency of crossing of SCI rats into the white zone of the PEAP box at week 7 was notably increased when compared to that of naïve rats. Delayed systemic DHA treatment significantly decreased the number of entries of SCI-rats into the white zone, when compared to that of vehicle-treated SCI rats. 
Data are presented as the mean \pm SEM. $\mathrm{N}=10-15$ per treatment group. One-way ANOVA Tukey-Kramer post hoc test were used for statistical analysis; ${ }^{*} \mathrm{p}<0.05,{ }^{*} \mathrm{p}<0.01, *^{*} \mathrm{p}<0.001$ vs vehicle. 
Supplementary Fig. 4
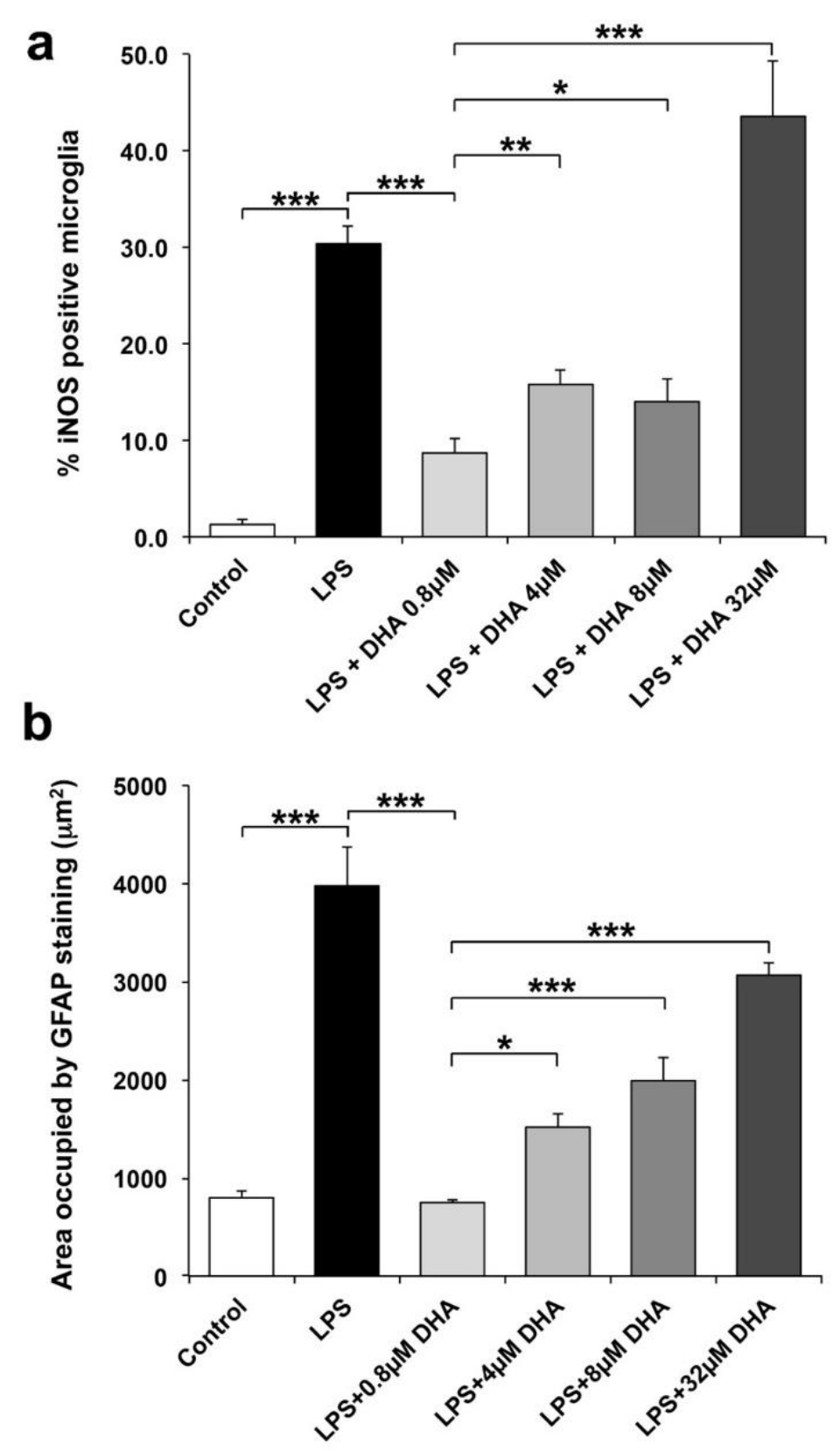

Supplementary Fig. 4. Analysis of the optimal concentration of DHA for modulation of LPSinduced microglial and astrocyte activation in vitro. (a) Microglial cell cultures treated with $10 \mu \mathrm{g} / \mathrm{ml}$ LPS over a period of 4 hours had a significantly higher percentage of microglia expressing iNOS compared to that of control cultures. Simultaneous treatment with DHA at four different concentrations revealed that $0.8 \mu \mathrm{M}$ DHA had the highest efficacy in reducing the number of iNOS-positive microglia. 
(b) Cultures treated with $10 \mu \mathrm{g} / \mathrm{ml}$ LPS for 4 hours had a significantly higher number GFAP immunoreactive astrocytes, when compared to control astrocyte cultures. Treatment with DHA at concentration of $0.8 \mu \mathrm{M}$ applied simultaneously during the 4 hours of LPS exposure, significantly reduced the GFAP immunoreactivity of the astrocytes, when compared to that of LPS treated only astrocyte cultures. Data are presented as the mean \pm SEM. $\mathrm{N}=3$ biological replicates/n=9 technical replicates per treatment group. One-way ANOVA and Tukey-Kramer post hoc test were used for statistical analysis; $* \mathrm{p}<0.05, * * \mathrm{p}<0.01, * * * \mathrm{p}<0.001$ vs LPS $+0.8 \mu \mathrm{M}$ DHA. $* * * \mathrm{p}<0.001$ of LPS vs control or LPS $+0.8 \mu \mathrm{M}$ DHA. 


\section{Supplementary Fig. 5}

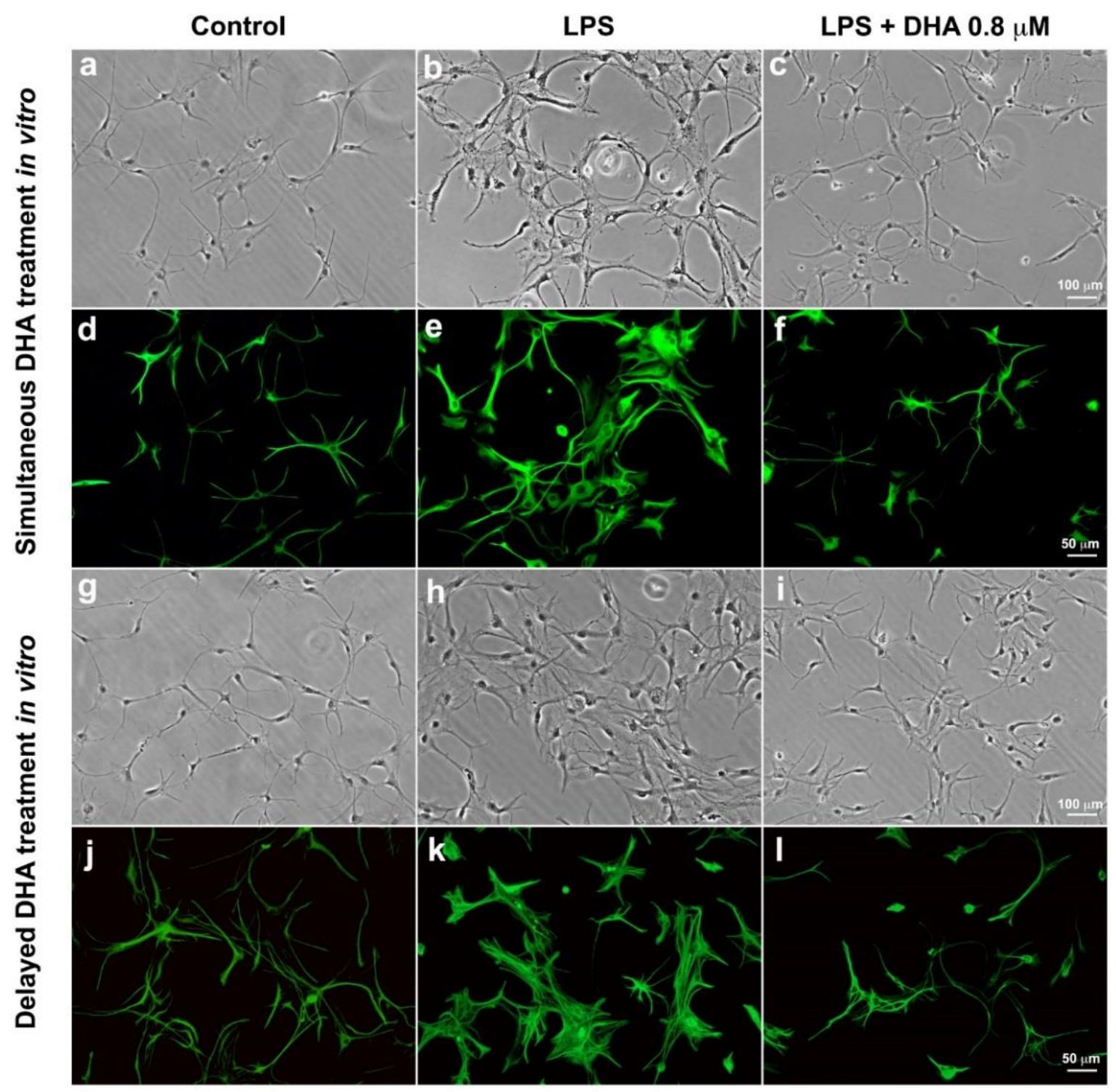

Supplementary Fig. 5. DHA attenuates LPS-induced astrocytes activation in vitro. Phase-contrast

live images of (a) control astrocytes, (b) LPS-treated astrocytes, and (c) astrocytes treated with $0.8 \mu \mathrm{M}$ DHA during simultaneous exposure to LPS for 4 hours revealed that DHA treatment led to the preservation of the non-reactive morphology of astrocytes manifested in the control cultures, characterized by slender cell processes and small cell bodies. Fluorescent images of (d) control, (e) LPStreated, and (f) astrocytes treated with $0.8 \mu \mathrm{M}$ DHA simultaneously during 4 hours of LPS stimulation, showed that DHA treatment reduced the high GFAP staining intensity of astrocytes (indicator of their 
activation) seen in LPS-treated astrocyte cultures. Bright field live images of (g) control, (h) LPS-treated, and (i) pre-activated for 4 hours with LPS astrocytes, which received a delayed DHA treatment at the concentration of $0.8 \mu \mathrm{M}$ for 4 additional hours post-activation, demonstrated that DHA markedly reversed the LPS-induced activated morphology of astrocytes nearly to that of control cells. Fluorescent images of (j) control, (k) LPS-treated, and (l) DHA-treated pre-exposed to LPS astrocytes revealed that $0.8 \mu \mathrm{M}$ DHA treatment reduced the high GFAP staining intensity seen in the LPS-treated astrocyte cultures. 


\section{Supplementary Fig. 6}
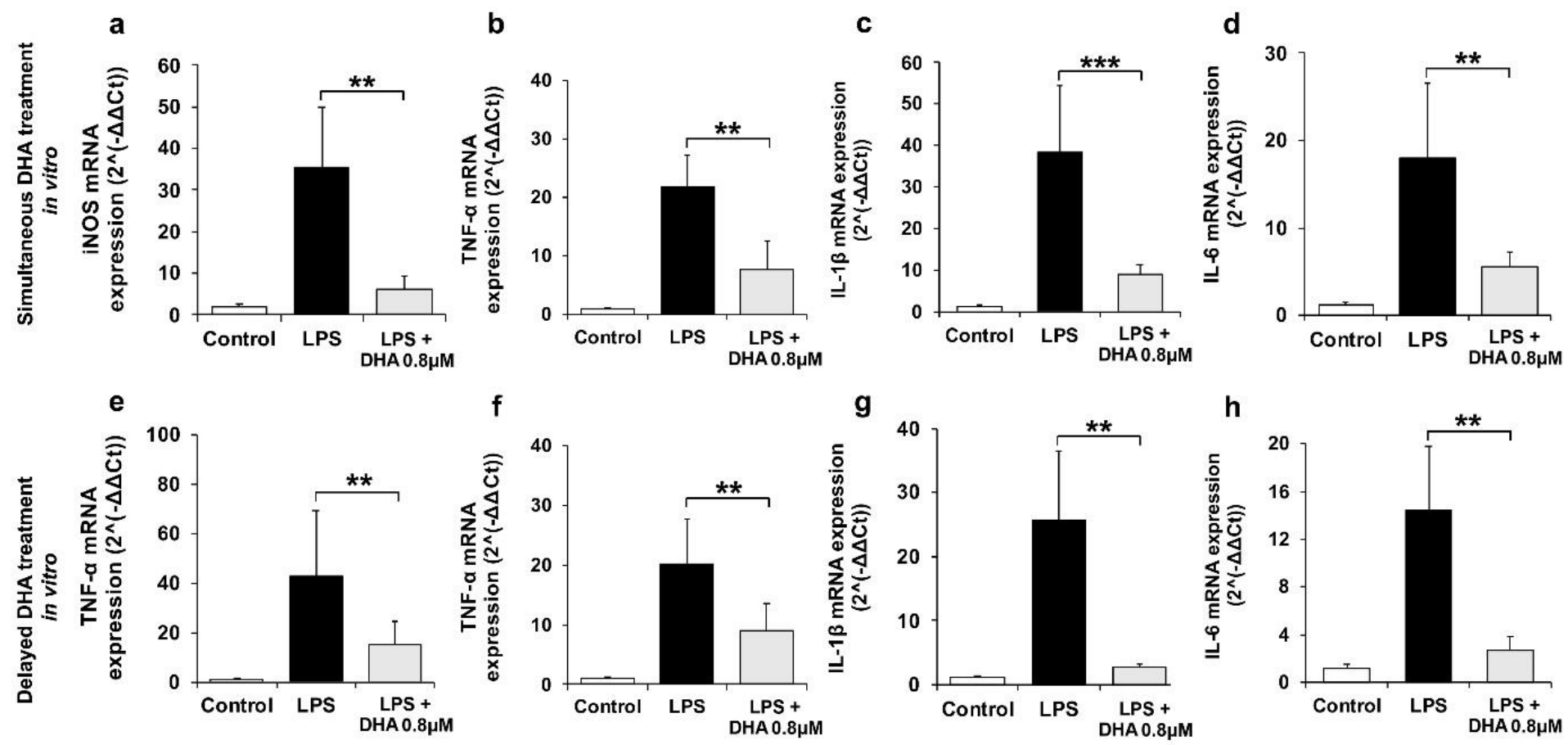

Supplementary Fig. 6. In vitro DHA treatment of LPS-activated astrocytes significantly decreased the relative mRNA expressions of proinflammatory mediators. Analysis of the qRT-PCR results for iNOS, TNF- $\alpha$, IL-1 $\beta$ and IL-6 mRNA expression levels demonstrated that: (a-d) simultaneous treatment of the astrocytes with $0.8 \mu \mathrm{M}$ DHA while exposed to LPS for 4 hours led to a significant reduction in the relative expressions of these proinflammatory genes, when compared to their expressions in astrocyte cultures stimulated with LPS only; (e-h) delayed treatment of astrocytes with $0.8 \mu \mathrm{M}$ DHA following 4 hours of pre-treatment with LPS also significantly lowered the relative expressions of proinflammatory genes, when compared to astrocyte cultures stimulated with LPS only. 
Supplementary Fig. 7

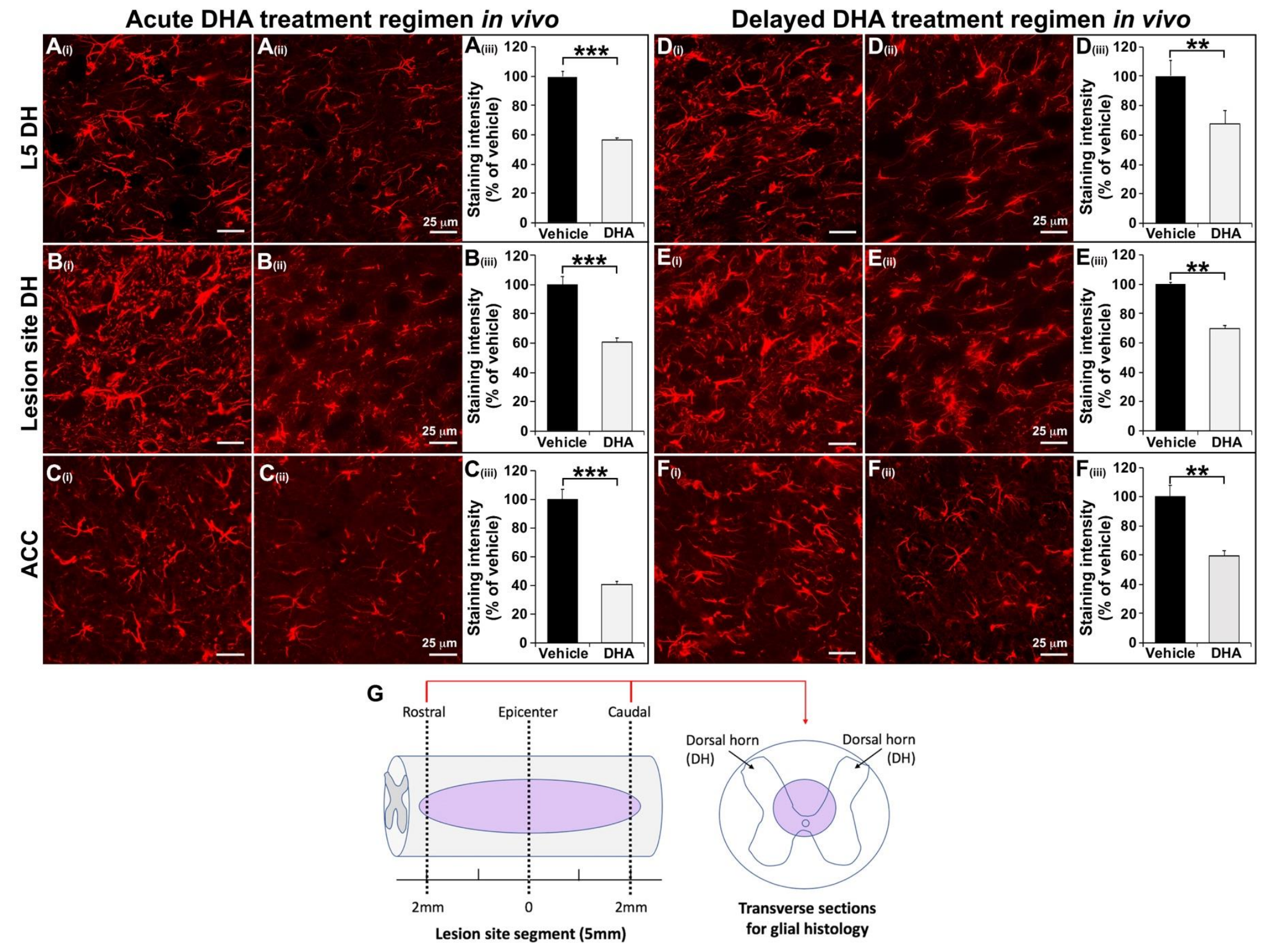


Supplementary Fig. 7: The effects of systemic DHA treatments on astrocyte activation. The acute regimen significantly decreased astrocyte activation (morphology/GFAP intensity) in dorsal horns (DH) of L5 ( $\left.\mathbf{A}_{(\mathbf{i} \text {-iii) }}\right)$ and epicenter $\left(\mathbf{B}_{(\mathrm{i}-\mathrm{iii})}\right)$ levels and the ACC $\left(\mathbf{C}_{(\mathrm{i} \text {-iii) }}\right)$, comparing to vehicle treatment. The delayed regimen also reduced astrocyte activation in L5 dorsal horns $\left(\mathbf{D}_{(\mathrm{i}-\mathrm{iii})}\right)$, lesion site dorsal horns $\left(\mathbf{E}_{(\mathbf{i}-}\right.$ iii), and ACC $\left(\mathbf{F}_{(\mathbf{i}-i i i)}\right)$, in contrast to that of vehicle-treated rats. N=5-6 per group. Mann-Whitney test was used for statistical analysis. $(\mathrm{G})$ is a schematic drawing of the lesion site segment from where lesion site DHs were analyzed for GFAP staining. $* \mathrm{p}<0.05, * * \mathrm{p}<0.01, * * * \mathrm{p}<0.001$ vs vehicle. 
Supplementary Fig. 8

DHA treatment starting $\mathbf{3 0}$ min post $\mathrm{SCl}$

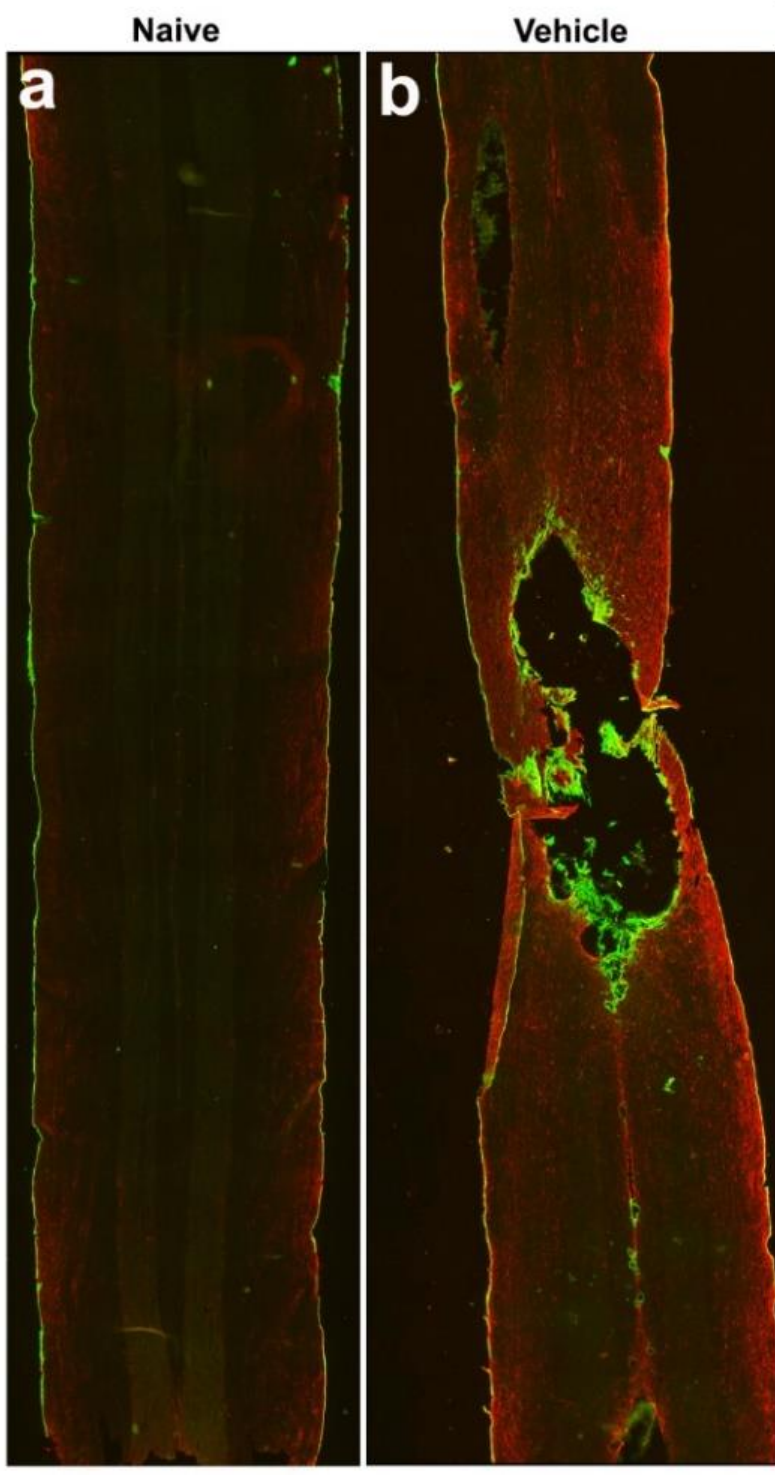

DHA treatment starting week 4 post $\mathrm{SCI}$ Vehicle DHA

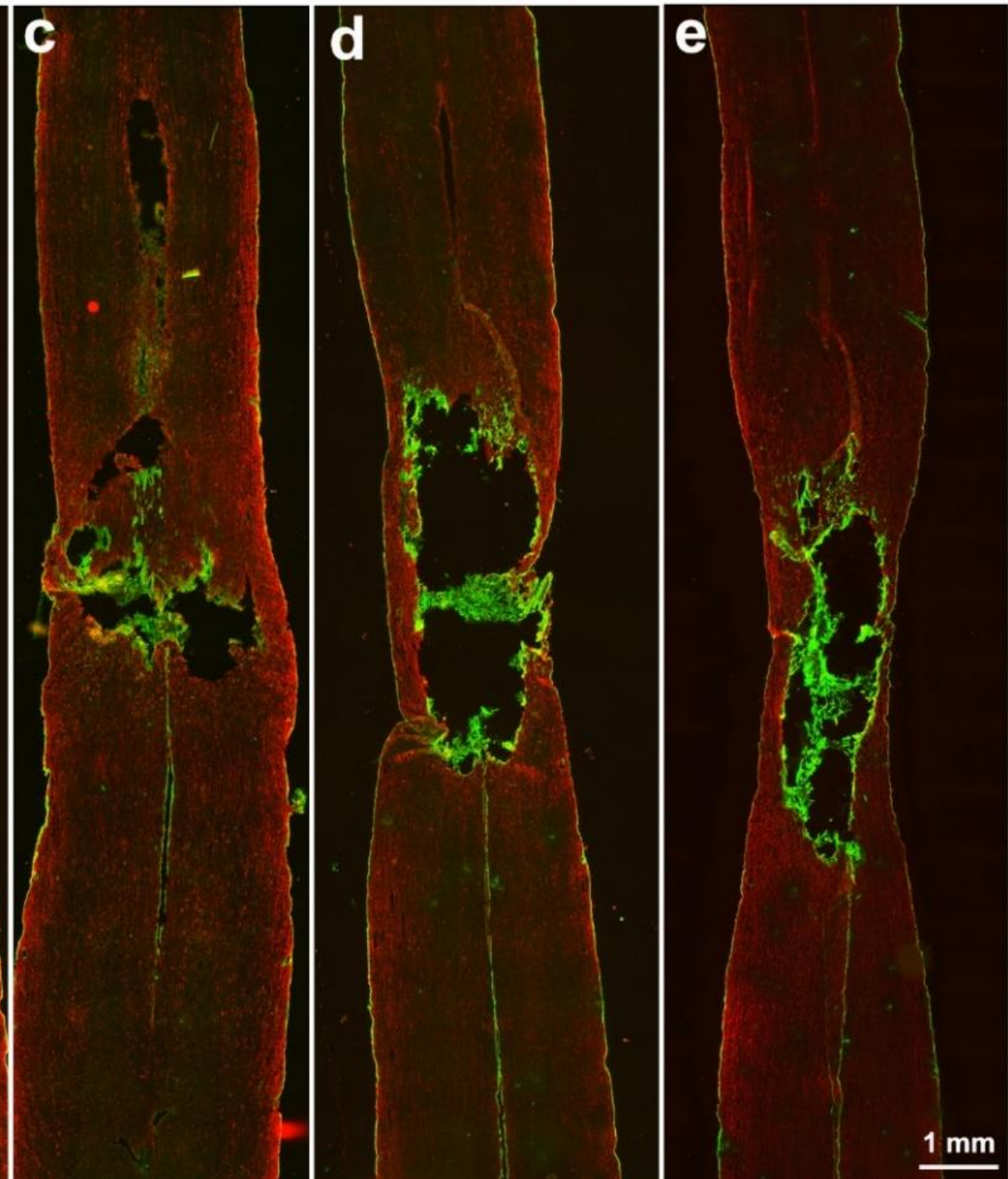


Supplementary Fig. 8. Lesion cavities at week 6 and week 7 following SCI in vivo. Representative fluorescent images of $15 \mathrm{~mm}$ spinal cord segments containing the lesion cavity stained with GFAP (red) and laminin (green) revealed that: (a) Naïve spinal cord is lacking laminin staining due to the absence of lesion cavity and the staining intensity of GFAP is very low due to the absence of astrocyte activation in the healthy cord. (b) The lesion cavity at week 6 of SCI rats receiving vehicle treatment is evident from the pronounced accumulation of astrocytes (GFAP) and extracellular matrix (laminin) around the injury site. (c) Intravenous DHA treatment administered 30 minutes following SCI surgery and every 3 days thereafter until the end of the study, appeared to reduce the cavity size after the injury and the accumulation of astrocytes and extracellular matrix around the site of impact. (d) The lesion cavity at week 7 of SCI rats receiving vehicle treatment seemed similar to that of vehicle-treated SCI rat at week 6. (e) Chronic administration of DHA treatment did not appear to reduce the cavity size as compared to that of the vehicle-treated group. $\mathrm{N}=2$ per treatment group. 
Supplementary Fig. 9

\section{DHA treatment starting $\mathbf{3 0}$ min post $\mathrm{SCI}$}

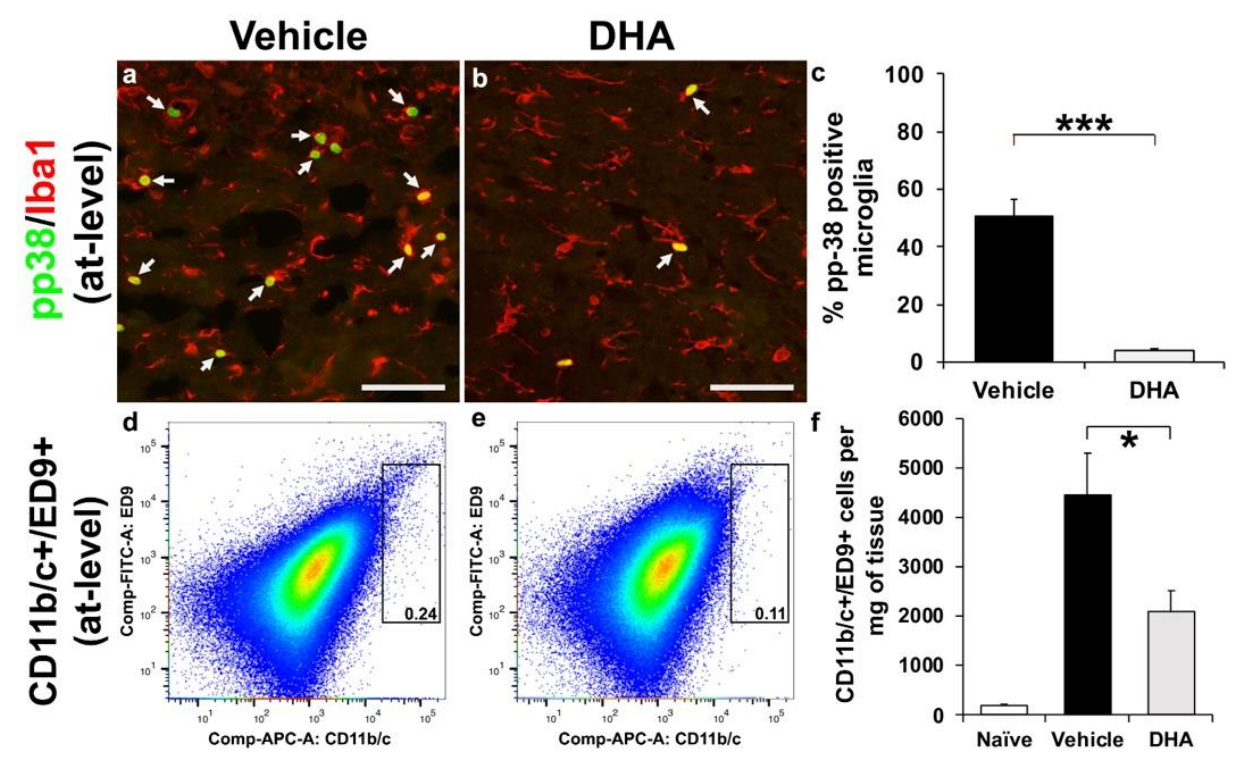

\section{DHA treatment starting week 4 post SCI}

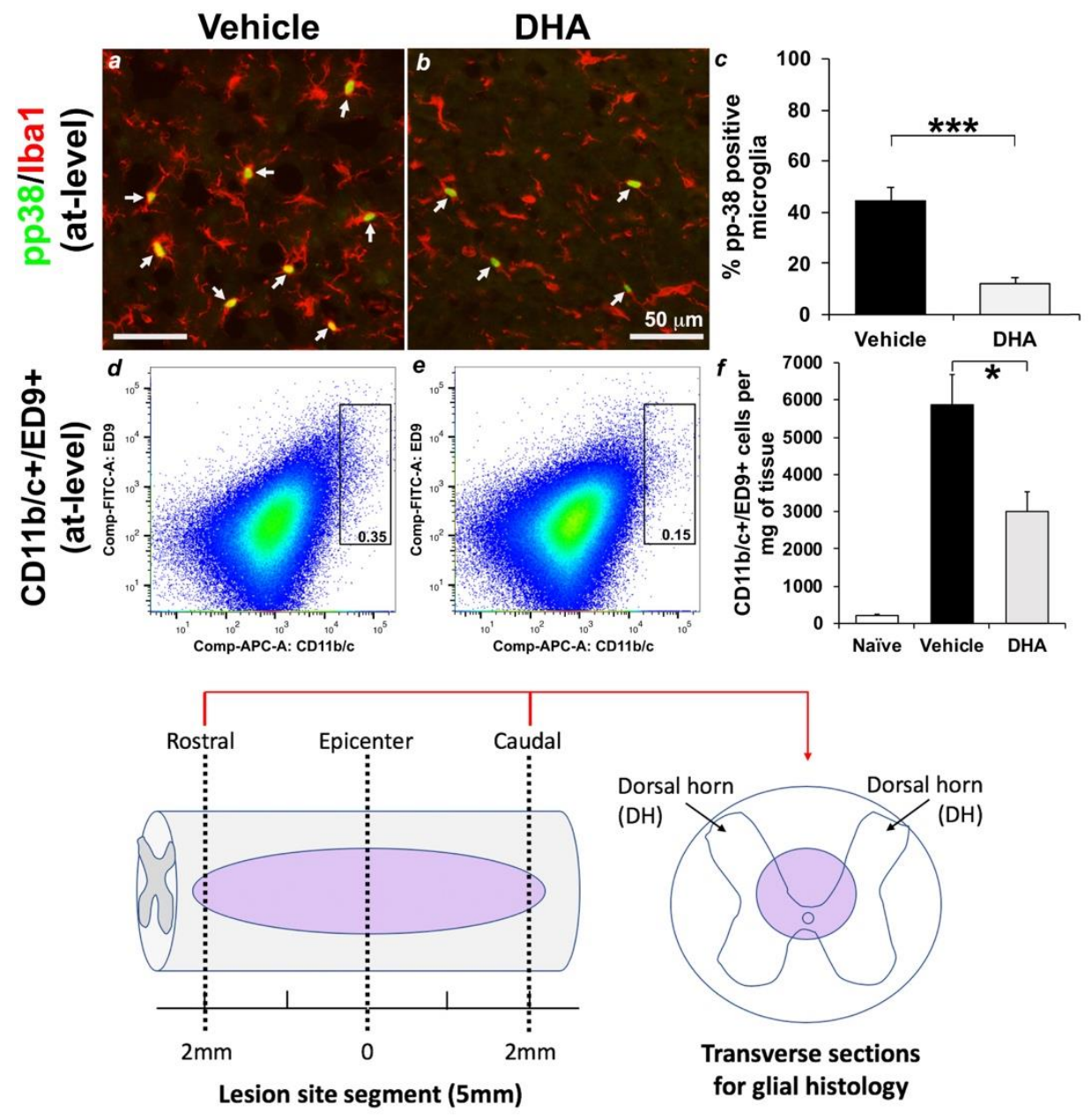




\section{Supplementary Fig. 9. The effects of systemic DHA treatment on SCI-induced microgliosis at the}

lesion site of SCI rats at week 6 and week 7 post-surgery. DHA treatment administered 30 minutes following SCI and every 3 days thereafter significantly reduced the expression of pp-38 by microglial cells (b\&c) and significantly decreased the number of CD11b/c and ED9 double-positive microglial cells per mg spinal cord tissue (e\&f), when compared to those of vehicle-treated SCI rat (a\&c and d\&f). Similarly, DHA treatment administered 4 weeks following SCI and every 3 days thereafter, significantly reduced the expression of pp-38 by microglial cells $(b \& c)$ and significantly decreased the number of CD11b/c and ED9 double-positive microglial cells ( $e \& f)$, when compared to those of vehicle-treated SCI rats $(a \& c$ and $d \& f)$. Data are presented as the mean $\pm \mathrm{SEM} ; \mathrm{N}=4-5$ per treatment group. Wilcoxon-MannWhitney and unpaired student's $t$-test were used for statistical analysis. The bottom panel is a schematic drawing of the lesion site segment from where lesion site DHs were analyzed for GFAP staining. $* \mathrm{p}<0.05, * * \mathrm{p}<0.01, * * * \mathrm{p}<0.001$ vs vehicle. 


\section{Supplementary Discussion}

The intravenous dose of FFA DHA used in the current studies is $250 \mathrm{nmol} / \mathrm{kg}$ in a volume of $5 \mathrm{ml} / \mathrm{kg}$. If assuming the average body weight of young adult rats is $250 \mathrm{~g}$, then the concentration of DHA in the injected bolus solution is $62.5 \mu \mathrm{M}$. We do not know how this injected FFA DHA concentration would be translated into the plasma FFA DHA concentration and how long this plasma DHA concentration would last. A recent study conducted in humans showed that repeated weekly 4-hour intravenous infusion of omega-3 fatty acids (fish oil formulation) including DHA led to significantly increased FFA DHA levels in the plasma (7-fold post-infusion verse pre-infusion) after the first infusion, which was highly repeatable across each of the later infusions up to 24 weeks [3]. We expect that the plasma FFA DHA concentration in our studies would have significantly increased after the first intravenous injection, and this increase would have been maintained across each of the later injections (every 3 days). Future studies are needed to investigate the pharmacokinetic studies for DHA, which is beyond the scope of the current studies.

We injected a bolus of FFA DHA solution at $62.5 \mu \mathrm{M}$ as stated above. We do not know the exact percentage of this $62.5 \mu \mathrm{M}$ DHA that would have reached the brain and spinal cord; that would require further investigations that are beyond the scope of the current studies. It has been demonstrated in the literature that $<1 \%$ of radiolabelled omega- 6 arachidonic acid, when given intravenously, reached rat brains (with an intact blood-brain barrier) [6]. It has also been shown that about $1 \%$ of radiolabelled DHA was found to be incorporated in rat brain lipids after an intravenous infusion [9]. Therefore, by analogy, it is likely that in our studies a small proportion of the injected FFA DHA entered the CNS and was incorporated rapidly in phospholipids (may be higher than 1\%, considering the blood-brain barrier is compromised following spinal cord injury). If assuming $1 \%$ of injected FFA DHA reached the CNS, the 
concentration of incorporated FFA DHA in the CNS would be around $0.625 \mu \mathrm{M}$. The FFA DHA in brain has been reported to be as low as $1.3 \mu \mathrm{M}$ [2]. Therefore, the assumed $0.625 \mu \mathrm{M}$ concentration of DHA is likely to remain in a physiological range and should be also compatible with the affinity of this fatty acid for targets such as ion channels and RXR receptors, which may be activated by FFA DHA [7]. In our in vitro experiments, we examined $0.8,4$, and $8 \mu \mathrm{M}$ FFA DHA concentrations, and found that DHA at all three concentrations was able to reduce microglial and astrocyte activation (Supplementary Fig. 4), with $0.8 \mu \mathrm{M}$ being found as the optimal concentration. Therefore, the in vivo FFA DHA concentration in the CNS as assumed above would be in line with the in vitro concentrations used in the current studies.

Based on the findings from the human study involving repeated weekly intravenous infusion of omega3 fatty acids including DHA as discussed above [3], we would expect that following the initial bolus FFA DHA injection in our studies, the FFA DHA levels in the plasma would have increased more than 7-fold, which was seen with the human study. This is because the human study used emulsion form of omega-3 fatty acids which would require an extra step of hydrolysis to release FFA DHA. We would also expect that a peak plasma concentration would have been reached very quickly after each intravenous injection, as previous evidence showed that about $1 \%$ of the dose of radiolabelled DHA injected intravenously was found in the rat brain 15 minutes after the injection. Future studies would need to establish the concentration range of DHA in the plasma including the peak plasma concentration following one DHA injection and before the next injection. In our studies, we used a repeated intravenous administration regime, i.e. every 3-day intervals, which would be a favourable regime in patients in the acute and subacute periods after SCI. We would anticipate that our regime would have maintained a constant plasma supply of FFA DHA to be incorporated into the CNS at a physiological range as discussed above. It is important to note that only FFA DHA in the plasma would be able to cross the blood-brain barrier 
and reach the CNS, so that CNS cells such as neurons, microglia and astrocytes can take up FFA DHA using general FFA uptake mechanisms or via receptor binding [3].

\section{Supplementary references:}

[1] Basso DM, Beattie MS, Bresnahan JC. Graded histological and locomotor outcomes after spinal cord contusion using the NYU weight-drop device versus transection. Experimental neurology 1996;139(2):244-256.

[2] Contreras MA, Greiner RS, Chang MC, Myers CS, Salem N, Jr., Rapoport SI. Nutritional deprivation of alpha-linolenic acid decreases but does not abolish turnover and availability of unacylated docosahexaenoic acid and docosahexaenoyl-CoA in rat brain. J Neurochem 2000;75(6):2392-2400.

[3] Eltweri AM, Thomas AL, Fisk HL, Arshad A, Calder PC, Dennison AR, Bowrey DJ. Plasma and erythrocyte uptake of omega-3 fatty acids from an intravenous fish oil based lipid emulsion in patients with advanced oesophagogastric cancer. Clin Nutr 2017;36(3):768-774.

[4] Hall JC, Priestley JV, Perry VH, Michael-Titus AT. Docosahexaenoic acid, but not eicosapentaenoic acid, reduces the early inflammatory response following compression spinal cord injury in the rat. $\mathbf{J}$ Neurochem 2012;121(5):738-750.

[5] Huang WL, King VR, Curran OE, Dyall SC, Ward RE, Lal N, Priestley JV, Michael-Titus AT. A combination of intravenous and dietary docosahexaenoic acid significantly improves outcome after spinal cord injury. Brain 2007;130(Pt 11):3004-3019.

[6] Jones CR, Arai T, Bell JM, Rapoport SI. Preferential in vivo incorporation of [3H]arachidonic acid from blood in rat brain synaptosomal fractions before and after cholinergic stimulation. J Neurochem 1996;67(2):822-829.

[7] King VR, Huang WL, Dyall SC, Curran OE, Priestley JV, Michael-Titus AT. Omega-3 fatty acids improve recovery, whereas omega-6 fatty acids worsen outcome, after spinal cord injury in the adult rat. J Neurosci 2006;26(17):4672-4680. 
[8] Liu ZH, Yip PK, Adams L, Davies M, Lee JW, Michael GJ, Priestley JV, Michael-Titus AT. A Single Bolus of Docosahexaenoic Acid Promotes Neuroplastic Changes in the Innervation of Spinal Cord Interneurons and Motor Neurons and Improves Functional Recovery after Spinal Cord Injury. J Neurosci 2015;35(37):12733-12752.

[9] Rapoport SI, Chang MC, Spector AA. Delivery and turnover of plasma-derived essential PUFAs in mammalian brain. J Lipid Res 2001;42(5):678-685.

[10] Saghaei M. Random allocation software for parallel group randomized trials. BMC Med Res Methodol 2004;4:26.

[11] Varone A, Knight D, Lesage S, Vollrath F, Rajnicek AM, Huang W. The potential of Antheraea pernyi silk for spinal cord repair. Sci Rep 2017;7(1):13790. 


\section{The ARRIVE Guidelines Checklist}

\section{Animal Research: Reporting In Vivo Experiments}

Carol Kilkenny ${ }^{1}$, William J Browne ${ }^{2}$, Innes C Cuthill ${ }^{3}$, Michael Emerson ${ }^{4}$ and Douglas G Altman ${ }^{5}$

${ }^{1}$ The National Centre for the Replacement, Refinement and Reduction of Animals in Research, London, UK, ${ }^{2}$ School of Veterinary Science, University of Bristol, Bristol, UK, ${ }^{3}$ School of Biological Sciences, University of Bristol, Bristol, UK, ${ }^{4}$ National Heart and Lung Institute, Imperial College London, UK, ${ }^{5}$ Centre for Statistics in Medicine, University of Oxford, Oxford, UK.

\begin{tabular}{|c|c|c|c|}
\hline & ITEM & RECOMMENDATION & $\begin{array}{l}\text { Section/ } \\
\text { Paragraph }\end{array}$ \\
\hline Title & 1 & $\begin{array}{l}\text { Provide as accurate and concise a description of the content of the article } \\
\text { as possible. }\end{array}$ & Title page \\
\hline Abstract & 2 & $\begin{array}{l}\text { Provide an accurate summary of the background, research objectives, } \\
\text { including details of the species or strain of animal used, key methods, } \\
\text { principal findings and conclusions of the study. }\end{array}$ & $\begin{array}{l}\text { Main text } \\
\text { Page } 1\end{array}$ \\
\hline \multicolumn{4}{|l|}{ INTRODUCTION } \\
\hline Background & 3 & $\begin{array}{l}\text { a. Include sufficient scientific background (including relevant references to } \\
\text { previous work) to understand the motivation and context for the study, } \\
\text { and explain the experimental approach and rationale. } \\
\text { b. Explain how and why the animal species and model being used can } \\
\text { address the scientific objectives and, where appropriate, the study's } \\
\text { relevance to human biology. }\end{array}$ & $\begin{array}{l}\text { Main text } \\
\text { Page 2-3 } \\
\text { Main text } \\
\text { Page } 3\end{array}$ \\
\hline Objectives & 4 & $\begin{array}{l}\text { Clearly describe the primary and any secondary objectives of the study, or } \\
\text { specific hypotheses being tested. }\end{array}$ & $\begin{array}{l}\text { Main text } \\
\text { Page } 3\end{array}$ \\
\hline \multicolumn{4}{|l|}{ METHODS } \\
\hline Ethical statement & 5 & $\begin{array}{l}\text { Indicate the nature of the ethical review permissions, relevant licences (e.g. } \\
\text { Animal [Scientific Procedures] Act 1986), and national or institutional } \\
\text { guidelines for the care and use of animals, that cover the research. }\end{array}$ & $\begin{array}{l}\text { Main text } \\
\text { Page } 4\end{array}$ \\
\hline Study design & 6 & $\begin{array}{l}\text { For each experiment, give brief details of the study design including: } \\
\text { a. The number of experimental and control groups. } \\
\text { b. Any steps taken to minimise the effects of subjective bias when } \\
\text { allocating animals to treatment (e.g. randomisation procedure) and when } \\
\text { assessing results (e.g. if done, describe who was blinded and when). } \\
\text { c. The experimental unit (e.g. a single animal, group or cage of animals). } \\
\text { A time-line diagram or flow chart can be useful to illustrate how complex } \\
\text { study designs were carried out. }\end{array}$ & $\begin{array}{l}\text { Suppl. M\&M: } \\
\text { Pages 2-3 } \\
\text { Page } 4 \\
\text { Pages } 2-3 \\
\text { Main text } \\
\text { Fig. } 1\end{array}$ \\
\hline $\begin{array}{l}\text { Experimental } \\
\text { procedures }\end{array}$ & 7 & $\begin{array}{l}\text { For each experiment and each experimental group, including controls, } \\
\text { provide precise details of all procedures carried out. For example: } \\
\text { a. How (e.g. drug formulation and dose, site and route of administration, } \\
\text { anaesthesia and analgesia used [including monitoring], surgical } \\
\text { procedure, method of euthanasia). Provide details of any specialist } \\
\text { equipment used, including supplier(s). } \\
\text { b. When (e.g. time of day). } \\
\text { c. Where (e.g. home cage, laboratory, water maze). } \\
\text { d. Why (e.g. rationale for choice of specific anaesthetic, route of } \\
\text { administration, drug dose used). }\end{array}$ & $\begin{array}{l}\text { Main text: } \\
\text { Pages 4-8 } \\
\text { Pages 4-8 } \\
\text { Pages 4-8 } \\
\text { Pages 4-8 }\end{array}$ \\
\hline $\begin{array}{l}\text { Experimental } \\
\text { animals }\end{array}$ & 8 & $\begin{array}{l}\text { a. Provide details of the animals used, including species, strain, sex, } \\
\text { developmental stage (e.g. mean or median age plus age range) and } \\
\text { weight (e.g. mean or median weight plus weight range). } \\
\text { b. Provide further relevant information such as the source of animals, } \\
\text { international strain nomenclature, genetic modification status (e.g. } \\
\text { knock-out or transgenic), genotype, health/immune status, drug or test } \\
\text { naïve, previous procedures, etc. }\end{array}$ & $\begin{array}{l}\text { Main text: } \\
\text { Page } 4\end{array}$ \\
\hline
\end{tabular}




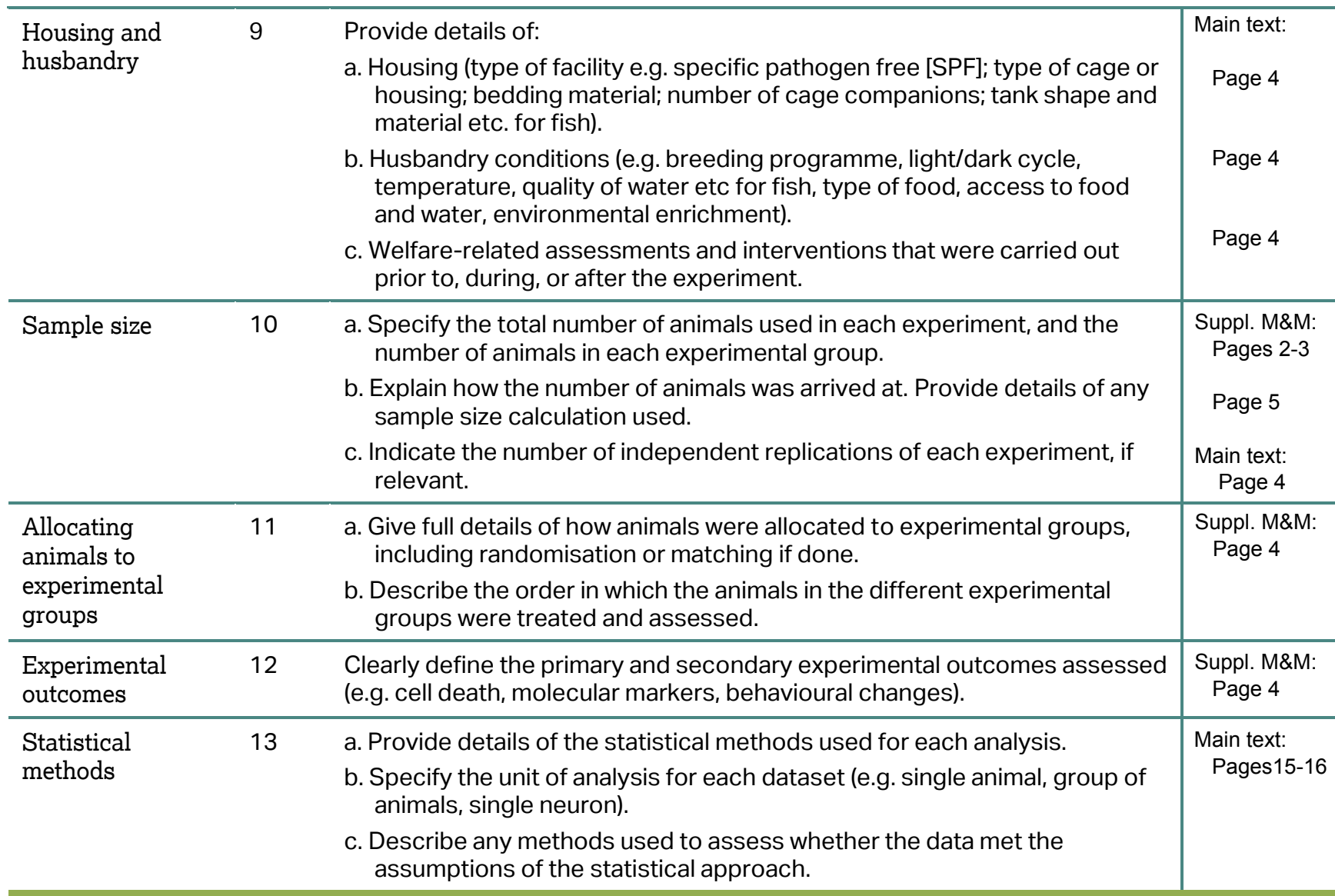

\begin{tabular}{|c|c|c|}
\hline \multicolumn{3}{|l|}{ RESULTS } \\
\hline Baseline data & 14 & $\begin{array}{l}\text { For each experimental group, report relevant characteristics and health } \\
\text { status of animals (e.g. weight, microbiological status, and drug or test naïv } \\
\text { prior to treatment or testing. (This information can often be tabulated). }\end{array}$ \\
\hline $\begin{array}{l}\text { Numbers } \\
\text { analysed }\end{array}$ & 15 & $\begin{array}{l}\text { a. Report the number of animals in each group included in each analysis. } \\
\text { Report absolute numbers (e.g. } 10 / 20 \text {, not } 50 \%^{2} \text { ). }\end{array}$ \\
\hline & & b. If any animals or data were not included in the analysis, explain why. \\
\hline
\end{tabular}

\begin{tabular}{lcll}
\hline $\begin{array}{l}\text { Outcomes and } \\
\text { estimation }\end{array}$ & 16 & $\begin{array}{l}\text { Report the results for each analysis carried out, with a measure of precision } \\
\text { (e.g. standard error or confidence interval). }\end{array}$ & $\begin{array}{c}\text { Main text: } \\
\text { Pages 17-25 } \\
\text { Finc }\end{array}$ \\
\hline Adverse events & 17 & $\begin{array}{l}\text { a. Give details of all important adverse events in each experimental group. } \\
\text { b. Describe any modifications to the experimental protocols made to } \\
\text { reduce adverse events. }\end{array}$ & $\begin{array}{c}\text { Suppl. M\&M: } \\
\text { Page } 4 \\
\text { N/A }\end{array}$ \\
\end{tabular}

\section{DISCUSSION}

Interpretation/

scientific

implications
18
a. Interpret the results, taking into account the study objectives and hypotheses, current theory and other relevant studies in the literature.
b. Comment on the study limitations including any potential sources of bias, any limitations of the animal model, and the imprecision associated with the results ${ }^{2}$.
c. Describe any implications of your experimental methods or findings for the replacement, refinement or reduction (the 3Rs) of the use of animals in research.

\begin{tabular}{lclll}
\hline $\begin{array}{l}\text { Generalisability/ } \\
\text { translation }\end{array}$ & 19 & $\begin{array}{l}\text { Comment on whether, and how, the findings of this study are likely to } \\
\text { translate to other species or systems, including any relevance to human } \\
\text { biology. }\end{array}$ & $\begin{array}{c}\text { Main text: } \\
\text { Pages 29-30 }\end{array}$ \\
\hline Funding & 20 & $\begin{array}{l}\text { List all funding sources (including grant number) and the role of the } \\
\text { funder(s) in the study. }\end{array}$ & $\begin{array}{c}\text { Main text: } \\
\text { Page 31 }\end{array}$ \\
\hline
\end{tabular}

